\title{
A Stoichiometric Calibration Method for Dual Energy Computed Tomography
}

\author{
Alexandra Bourque \\ Master ès Sciences \\ Medical Physics Unit \\ McGill University \\ Montréal, Québec, Canada
}

August 2013

A thesis submitted to the Faculty of Graduate Studies and Research in partial fulfillment of the requirements of the degree Master ès Sciences

(C)Alexandra Bourque 2013 
I would like to dedicate my research to Madeleine Bourque and whomever faced in any way the horrible disease that is cancer. By the mean of this research, I hope to help improve, even just a little, the treatment of cancer by radiation. 


\section{ACKNOWLEDGEMENTS}

First and foremost, I would like to thank my supervisor, Hugo Bouchard, for sharing with me his knowledge, his passion for research and his enthusiasm. He was present and open to discuss ideas, always giving me new goals to achieve. He not only fulfilled his role as a supervisor but also offered me support and guidance as a reference for questions I may have had about my future. I would also like to thank Jan Suentjens and Jean-François Carrier for their valuable advices and for letting me pursue my research project at the CHUM. On the teaching side at Mcgill; professor Ervin B. Podgorsak for his passion and his rigorousness. Finally, I wish to express my gratitude to my parents, Sylvie and Hélian, my sisters, Marie-Eve and Valérie, and to all my dear friends and fellow students who supported me through the duration of my studies. 


\begin{abstract}
While dose calculations are typically performed using a simplistic correspondence of Hounsfield units to electron density, recent developments in Dual Energy Computed Tomography (DECT) could provide significant improvements in characterizing human tissues in radiotherapy. The present study aims to establish and validate a DECT mathematical formalism and evaluate its accuracy in characterizing tissue in terms of electron density and effective atomic number for radiotherapy applications. A relationship between the effective atomic number and the mean excitation energy is proposed to calculate parameters for proton and heavy ion therapy, as the stopping power. A novel definition of the effective atomic number is also developed. A Gammex 467 phantom is scanned at different energy couples with a Siemens SOMATOM Definition Flash in order to apply the calibration-based formalism. The root mean square errors on the extracted parameters for the 100-140/Sn energy couple are $0.46 \%$ for the relative electron density, $2.5 \%$ for the effective atomic number, $4.9 \%$ for the mean excitation energy and $0.67 \%$ for the proton stopping power. The accuracy of such results presents many advantages in the field of radiation therapy. Thus, this work is expected to bring significant improvements in Monte Carlo dose calculation in future clinical applications.
\end{abstract}




\begin{abstract}
ABRÉGÉ
Bien que les calculs de dose soient généralement effectués en utilisant une correspondance directe entre les unités Hounsfield et la densité électronique, les développements récents en tomodensitométrie à double énergie (DECT) pourraient apporter des améliorations significatives dans la caractérisation des tissus humains en radiothérapie . La présente étude vise à établir et valider un formalisme mathématique DECT et à évaluer sa précision dans la caractérisation des tissus en termes de densité électronique et de numéro atomique effectif pour les applications de radiothérapie. Une relation entre le nombre atomique effectif et l'énergie moyenne d'excitation est proposée afin de calculer des paramètres pour la thérapie par protons et par ions lourds, tel que le pouvoir d'arrêt. Une nouvelle définition du nombre atomique effectif est également développée. Un fantôme Gammex 467 est scanné à différents couples d'énergie avec un Siemens SOMATOM Definition Flash afin d'appliquer le formalisme. L'erreur moyenne quadratique des paramètres extraits pour le couple d'énergie 100-140/Sn sont $0.46 \%$ pour la densité électronique relative à l'eau, 2.5\% pour le nombre atomique effectif, $4.9 \%$ pour l'énergie d'excitation moyenne et $0.67 \%$ pour le pouvoir d'arrêt. L'exactitude de ces résultats présente de nombreux avantages en radiothérapie. Ainsi, ce travail pourrait apporter des améliorations significatives en calcul de dose Monte Carlo pour de futures applications cliniques.
\end{abstract}




\section{TABLE OF CONTENTS}

ACKNOWLEDGEMENTS ..................... . . iii

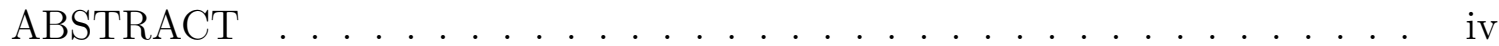

ABRÉGÉ ....................... . .

LIST OF ABBREVIATION ..................... ix

LIST OF TABLES . . . . . . . . . . . . . . . . . . . .

LIST OF FIGURES . . . . . . . . . . . . . . . . . . xi

1 Introduction . . . . . . . . . . . . . . . . . . . 1

1.1 Cancer and radiation therapy . . . . . . . . . . . . . . . 1

1.2 The role of Computed Tomography in radiation therapy . . . . . . 3

1.2.1 External and internal structures contours . . . . . . . . . . 3

1.2.2 Target localization . . . . . . . . . . . . . . . . 4

1.2.3 Patient coordinate system and treatment fields design . . . 5

1.2.4 Electron density data for treatment planning . . . . . . . . 6

1.3 Thesis objectives . . . . . . . . . . . . . . . 6

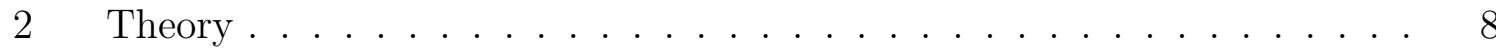

2.1 X-ray interactions with matter . . . . . . . . . . . . 8

2.1.1 Coherent scattering . . . . . . . . . . . . . . 99 9

2.1.2 Photoelectric effect . . . . . . . . . . . . . . . . . . . . . . . 9

2.1.3 Compton scattering . . . . . . . . . . . . . . . 13

2.1.4 Pair and triplet production . . . . . . . . . . . . . 15

2.2 Computed tomography . . . . . . . . . . . . . . . 16

2.2.1 X-rays production in CT . . . . . . . . . . . . . 17

2.2 .2 Detectors . . . . . . . . . . . . . . . 18

2.2 .3 Reconstruction . . . . . . . . . . . . . . . . 19

2.2.4 CT artefacts . . . . . . . . . . . . . . . 21 
2.3 Dual Energy Computed Tomography . . . . . . . . . . . . . . 21

3 Introduction to a stoichiometric calibration method for dual source computed tomography . . . . . . . . . . . . . . . . . 24

3.1 The calibration of HU in SECT . . . . . . . . . . . . . 24

3.2 Dual Energy Computed Tomography formalisms . . . . . . . . 25

3.2 .1 Rutherford et al. . . . . . . . . . . . . . . . . . 25

3.2 .2 Alvarez and Macovski . . . . . . . . . . . . . . . . . . 26

3.2 .3 Bazalova et al. . . . . . . . . . . . . . . . . . . . . . 27

3.3 Definitions of the effective atomic number . . . . . . . . . . 29

3.3 .1 Mayneord . . . . . . . . . . . . . . . . . . . 29

3.3 .2 Manohara et al. . . . . . . . . . . . . . . . . . . 29

3.3 .3 Taylor et al. . . . . . . . . . . . . . . . . 30

3.3 .4 The novel definition . . . . . . . . . . . . . . . 30

3.4 Objectives, results and impact of the scientific paper . . . . . . . 30

3.5 Contribution of authors . . . . . . . . . . . . . 32

3.6 Permissions . . . . . . . . . . . . . . . . . . . . 34

4 A stoichiometric calibration method for dual source computed tomography 35

4.1 Introduction . . . . . . . . . . . . . . . . 37

4.2 Theory . . . . . . . . . . . . . . . . . . . . 41

4.2.1 The SECT stoichiometric calibration of Schneider et al. . . 41

4.2.2 Electronic cross section parametrization . . . . . . . . . 43

4.2 .3 Mixtures . . . . . . . . . . . . . . . . . 45

4.2 .4 Effective atomic number $\ldots \ldots \ldots \ldots$

4.2.5 Mean excitation energy parametrization . . . . . . . . . 51

4.2.6 Extension of the SECT stoichiometric calibration . . . . . . 53

4.2.7 DECT stoichiometric calibration . . . . . . . . . . . 54

4.2 .8 Variance analysis . . . . . . . . . . . . . . . 57

4.2.9 Statistical uncertainties on experimental measurements . . 57

4.2.10 Statistical uncertainties on estimated parameters . . . . . 58

4.2.11 Non statistical uncertainties . . . . . . . . . . . . . 60

4.3 Materials and methods . . . . . . . . . . . . . . . . . . . 61

4.3.1 Theoretical framework . . . . . . . . . . . . . . . 61

4.3.2 Experimental measurements . . . . . . . . . . . . 63

4.4 Results and discussion . . . . . . . . . . . . . . . . 63

4.4.1 Theoretical self-consistency . . . . . . . . . . . . . 63 
4.4.2 Experimental measurements . . . . . . . . . . . . . 67

4.4.3 Heavy-ion range uncertainties . . . . . . . . . . . . . 72

4.5 Summary ...................... . . . 74

4.6 Acknowledgements .................... 76

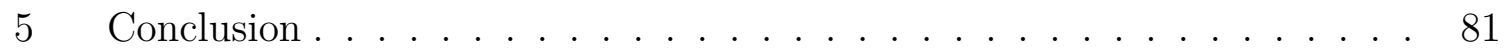

References ......................... 83 


\section{LIST OF ABBREVIATION}

CHUM Centre hospitalier de l'Université de Montréal

CT Computed Tomography

DECT Dual Energy Computed Tomography

DEI Dual energy index

DER Dual energy ratio

DSCT Dual Source Computed Tomography

EAN Effective atomic number

ED Electron density

FBP Filtered back projection

HU Hounsfield unit

ICRP International Commission on Radiological Protection

ICRU International Commission on Radiation Units \& Measurements

SECT Single Energy Computed Tomography 
4-1 Summary of the material parameters of the Gammex 467 phantom computed from mass densities and elemental compositions provided by the manufacturer (for reference use only). . . . . . . . . . . . . . 49

4-2 Summary of human tissue parameters computed from mass densities and elemental compositions recommended by the ICRP [42]. . . . . 50

4-3 Recommended values of mean excitation energies to determine the $I$-values of mixtures using Bragg's additivity rule [16] . . . . . . . . 52

4-4 Electron densities and elemental compositions of the Gammex 467 phantom materials (for reference use only). . . . . . . . . . 62

4-5 Machine parameters used during experimental acquisition. A $0.8 \mathrm{~mm}$ Sn filter is present in the $140 \mathrm{kVp}$ source (i.e., noted $140 / \mathrm{Sn} \mathrm{kVp}$ ) for the first and second energy couples. Note that these are not clinical protocols. ..................... 64

4-6 HU uncertainties for the SECT experimental calibration ( $1 \sigma$ level). . . 69

4-7 Impact on the standard deviation of the HU for filtered back projection (FBP) and SAFIRE reconstruction processes. The third level of iteration of SAFIRE is used. . . . . . . . . . . . . . . 770

4-8 Experimental RMS errors (in \%) of the calibration phantom's physical parameters determined experimentally. . . . . . . . . . 73

4-9 Experimental uncertainties on the electron density and the EAN for the $100-140 / \mathrm{Sn} \mathrm{kVp}$ energy couple. . . . . . . . . . . . 73 
1-1 Proportion of deaths due to cancer and other causes in Canada in 2009. 2

1-2 The classification of radiation with respect to their effect on matter. . 3

1-3 Illustrative example of a dose volume histogram. Courtesy of

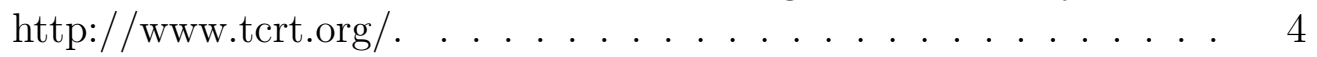

1-4 ICRU volumes defined prior to treatment planning [17] . . . . . . 5

1-5 Illustrative example of an HU-ED curve. Courtesy of Centre hospitalier de l'Université de Montréal. . . . . . . . . . . . . . . . . 7

2-1 Mass attenuation coefficient of water (a) and iron (b) as a function of the photon energy. Individual mass attenuation coefficients of equation 2.2 are also illustrated. Calculated from the XCOM database $[6] \ldots \ldots \ldots \ldots \ldots$

2-2 Region of relative predominance of the three main types of interactions of photons with matter $[32] \ldots \ldots \ldots \ldots$

2-3 Illustrative example of the photoelectric effect (left) and the Compton scattering (right). Courtesy of http://www.physics.hku.hk/. . . . 12

2-4 Fluorescence yield. Courtesy of http://xdb.lbl.gov/. . . . . . . . . . 12

2-5 Maximum and mean fraction of the incident photon energy transferred to the recoil electron $[32] \ldots \ldots \ldots \ldots$

2-6 Illustrative example of the nuclear pair production. Courtesy of http://www.radiologyschools.com/. . . . . . . . . . . 16

2-7 Third generation CT scanner. Courtesy of http://openi.nlm.nih.gov/. 17

2-8 X-ray tube. Courtesy of http://www.arpansa.gov.au/. . . . . . . 18 
2-9 Solid-state detectors [9]. . . . . . . . . . . . . . . . . . . . . . . 19

2-10 From the sinogram to the reconstruction. Courtesy of scien.stanford.edu. 20

2-11 Illustration of the Siemens SOMATOM Definition Flash dual source

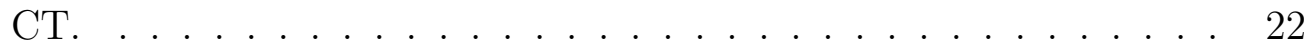

3-1 Maps of the extracted parameters for the Gammex-467 phantom. Note that the map of the relative proton stopping power is computed for a proton of kinetic energy equals to $216 \mathrm{MeV}$. . . . . . . . . . . 33

4-1 XCOM electron cross section averaged over the spectra of the Siemens SOMATOM Definition Flash DSCT as a function of the atomic number. . . . . . . . . . . . . . . .

4-2 Effective atomic number values calculated as a function energy from the XCOM database for ICRP tissues: adipose tissue (top left), cortical bone (top right), muscle (bottom left) and thyroid (bottom right). Note that scanner 1 refers to the Siemens SOMATOM Definition Flash and scanner 2 refers to the Philips Gemini GXL.

4-3 Parametrization of the ICRU mean excitation energy as a function of the EAN defined in this paper for ICRP tissues. . . . . . . . . 52

4-4 Behavior of the DEI (left) and DER (right) as a function the atomic number for elements. The data is obtained using XCOM cross sections averaged over the spectra. . . . . . . . . . . . . . 64

4-5 A theoretical comparison of residual HU errors between the model used by Schneider et al. and the present model. The spectra used to calculate theoretical $\mathrm{HU}$ values are a $80 \mathrm{kVp}$ spectrum (left) and a $140 \mathrm{kVp}$ spectrum (right). . . . . . . . . . . . . . . . 65

4-6 Relative errors in ED (left) and EAN (right) using the DECT formalism of theoretical HU values for 34 ICRP tissues. The error bars are non statistical uncertainties, i.e., $\Delta \rho_{e}$ is calculated with equation 4.50 and $\Delta Z_{\text {med }}$ is extracted from table $4-2 \ldots \ldots \ldots$

4-7 A theoretical comparison of the DEI between the ICRP tissues and the thyroid for the energy couple 100-140/Sn kVp. . . . . . . . . 68 
4-8 The ratios of calculated to known relative electron densities, EANs, mean excitation energies and proton stopping powers for the 100$140 / \mathrm{Sn} \mathrm{kVp}$ energy couple. Note that the proton kinetic energy is $216 \mathrm{MeV}$. The reported uncertainties include statistical and non statistical components. . . . . . . . . . . . . . . 72

4-9 Ideal uncertainty on the range of protons, helium ions and carbon ions in water as a function of the range of the beam. . . . . . . . 75 


\title{
Chapter 1 Introduction
}

\section{"Learn from yesterday, live for today, hope for tomorrow. The} important thing is not to stop questioning."

\author{
- Albert Einstein
}

\subsection{Cancer and radiation therapy}

The human body consists of trillions of cells, all of them carrying out different functions to form multiple organs and tissues. Each cell grows, divides and dies according to its own type and function. Lumps or tumours are formed when a cell starts dividing uncontrollably. There are two different types of tumours; benign and malignant. The word cancer is associated to a malignant tumour, which is defined by cells having the ability to invade other parts of the body through the bloodstream or the lymphatic system, creating metastases [43]. The name of a cancer is related to the organ where the cancer cells originate. For instance, breast cancer with lung metastases means that the cancer started in the breast and that cancerous cells from the breast invaded the lung.

As shown on figure 1-1, cancer is the leading cause of death in Canada in 2009 with $29.8 \%$ of all deaths [43]. This statistic is increasing because of the augmentation of the average life expectancy, obesity, lifestyle and environmental pollutants [18]. 


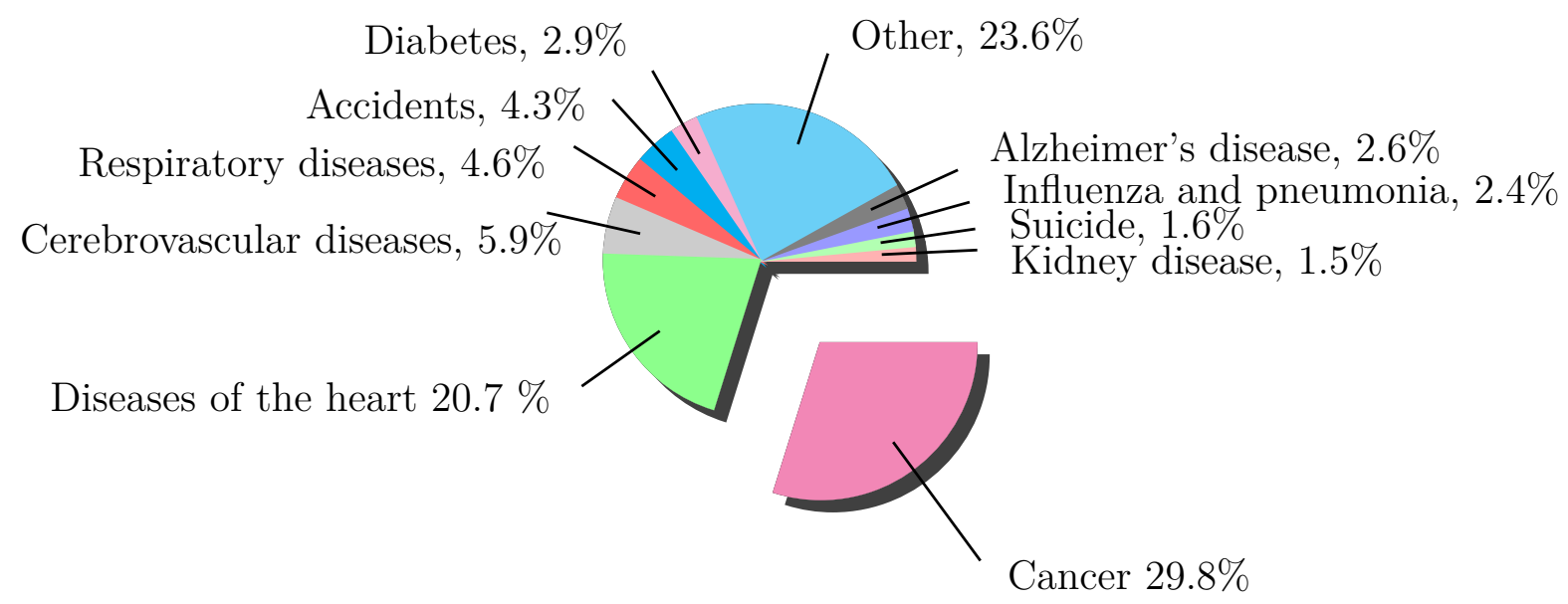

Figure 1-1: Proportion of deaths due to cancer and other causes in Canada in 2009.

Nowadays, there are many ways to treat cancer, such as chemotherapy, surgery and radiation therapy. Other adjuvant techniques may include hormonal therapy, targeted therapy and even cryosurgery [18].

Radiation therapy uses ionizing radiation to kill malignant cells by creating single or double strand breaks in their DNA. It may not only be used to cure cancer, but also for palliative purposes and non-malignant diseases treatments. As shown on figure 1-2, the classification of radiation is based on either the particle can directly or indirectly ionize a medium. In the case of indirectly ionizing radiation, the neutral particle first interacts with the matter to release a charged particle. A photon may eject an electron or a positron, while a neutron will release a proton or heavier ions. These particles are then considered directly ionizing radiation and will interacts with the matter through coulomb interactions [33], making their penetration power much smaller than neutral particles. The source of the radiation can either be external or internal and it can be produced using particle accelerators or radioisotopes. 


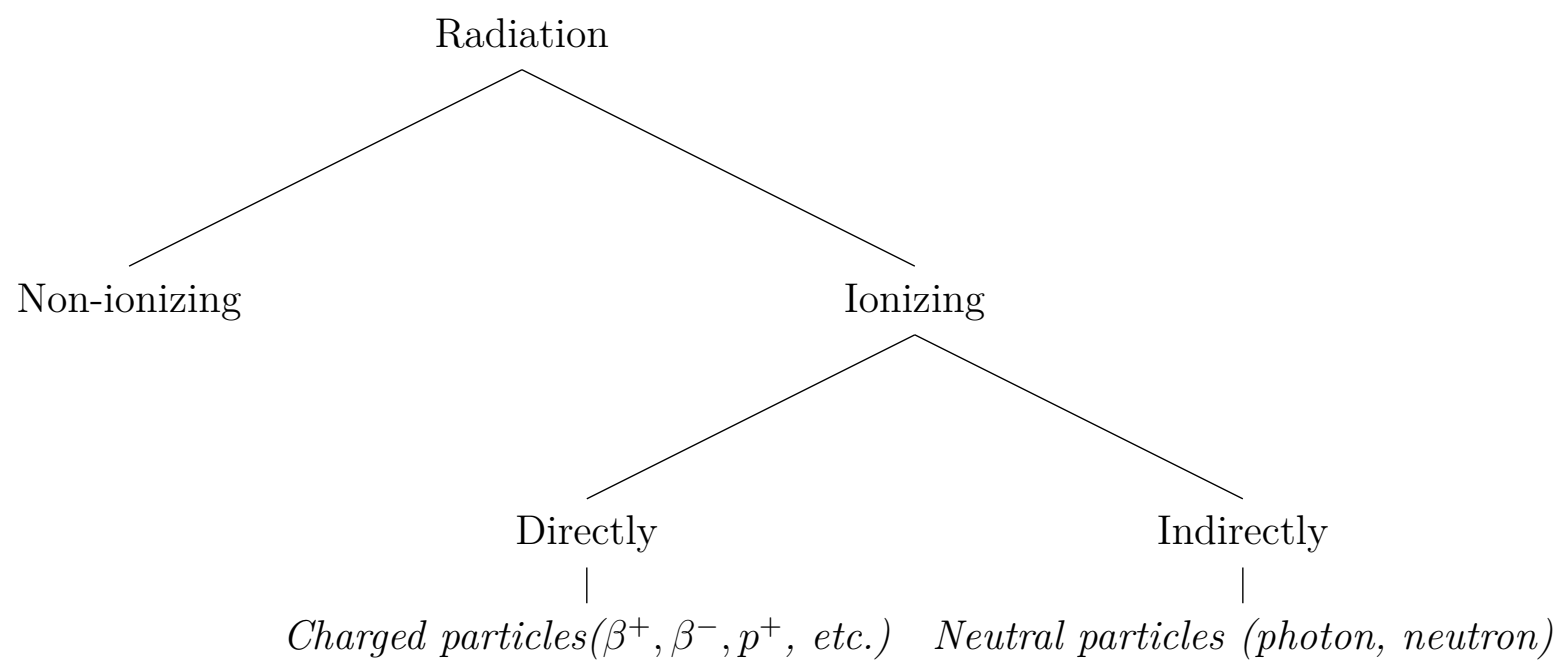

Figure 1-2: The classification of radiation with respect to their effect on matter.

\subsection{The role of Computed Tomography in radiation therapy}

Since its invention in 1971 by Sir Godfrey N. Hounsfield and Alan M. Cormack, Computed Tomography $(\mathrm{CT})$ is considered to be one of the most important innovation in the field of diagnostic radiology. From the first scan of a cerebral cyst to the first whole-body scan, this technology has now countless applications in medical and industrial imaging. Indeed, its capability to produce cross-sectional images makes it the perfect tool to obtain precise information and localization of the inner parts of an object. In 1979, for their contribution to the field of Medecine, Hounsfield and Cormack were awarded with a Nobel Prize. This section states the importance of conventional $\mathrm{CT}$ in radiation therapy.

\subsubsection{External and internal structures contours}

A radiation therapy patient is scanned with a flat couch using immobilization devices to assure that the patient will remain the same position during the series of 
treatments than during the imaging process. The CT data is analyzed by physicians to contour external and internal structures. While the skin of the patient is contoured for contour corrections, the internal structures include the tumour itself and organs that may be more sensitive to radiation, also known as organs at risk. These contours will help evaluating the dose delivered to these organs and the coverage of the tumour by using dose volume histograms (DVH). A DVH is defined as follows : as shown on figure 1-3, for any given point of volume $\mathrm{V} \%$ and dose $\mathrm{D} \mathrm{Gy}, \mathrm{V} \%$ of the volume of the organ will absorb at least the dose D Gy [33]. Is it an accurate way to compare and evaluate the quality of a plan.

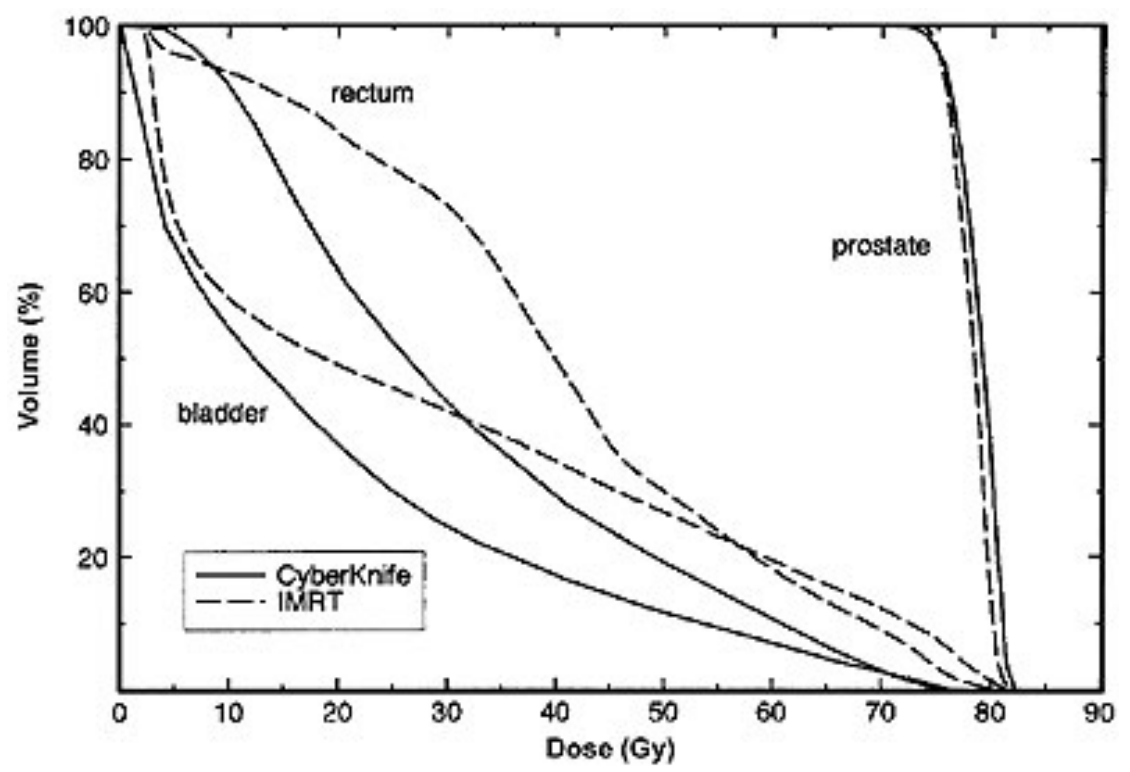

Figure 1-3: Illustrative example of a dose volume histogram. Courtesy of http://www.tcrt.org/.

\subsubsection{Target localization}

The volume of the visible tumour is not the only part that needs to be irradiated. In fact, ICRU report 50 [17] defines diverse volumes important for the treatment 
planning, as shown on figure 1-4. They are defined as follows: the gross tumour volume is simply the visible location of the tumour on the CT images, while the clinical target volume includes subclinical microscopic malignant disease. On the other hand, the planning target volume is more of a geometrical concept and take into account margins from variation in the delivery of the treatment by either the machine or set-up uncertainties. The treated volume is defined as the volume enclosed by a specific isodose surface that is considered to be appropriate to accomplish the goal of the treatment. Finally, the irradiated volume is the volume which receives a significant dose in comparison to the tolerance of normal tissues.

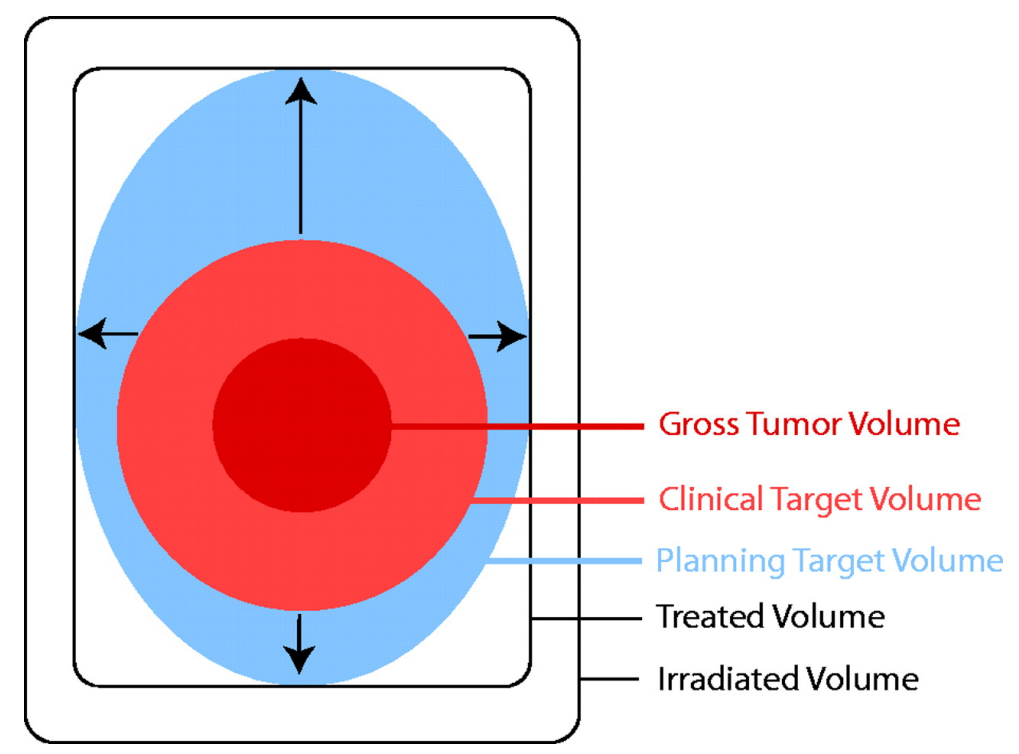

Figure 1-4: ICRU volumes defined prior to treatment planning [17].

\subsubsection{Patient coordinate system and treatment fields design}

Once the target is localized and the exact position of the patient is recorded, the treatment fields design is achieved by using the CT data in the treatment planning 
system. Consequently, the sparing of healthy tissues and the reduction of the margins are optimized [32].

\subsubsection{Electron density data for treatment planning}

Accurate dose calculation require knowledge of the tissue composition and density distribution in the patient. Indeed, photons going through media of higher or lower densities and atomic numbers will have an attenuation behaviour which is considerably different than assuming that the whole body of the patient is made of water, as for homogeneous dose calculations. Figure 1-5 shows an example of a calibration curve used to apply heterogeneities correction. It links the Hounsfield unit (HU), or also called CT number, to an electron density relative to water. A calibration phantom with plugs of various media of known chemical compositions and densities is scanned at the needed energies and the relative electron density of each plug is related to its average HU [40]. Thus, because each voxel of the patient's image is represented as a HU, the respective electron density will be assigned and the variation of the tissues composition will be taken into account. The number of segments is subject to change and the trends of the curves are energy and scanner dependent.

\subsection{Thesis objectives}

With the advent of Dual Energy Computed Tomography (DECT) machines in radiation therapy departments, it has become substantial to evaluate the advantages of such a technology in radiotherapy. Therefore, the objective of this work is to establish a calibration-based formalism as a reference to explore the applications of spectral imaging Computed Tomography in radiation therapy by extracting the 


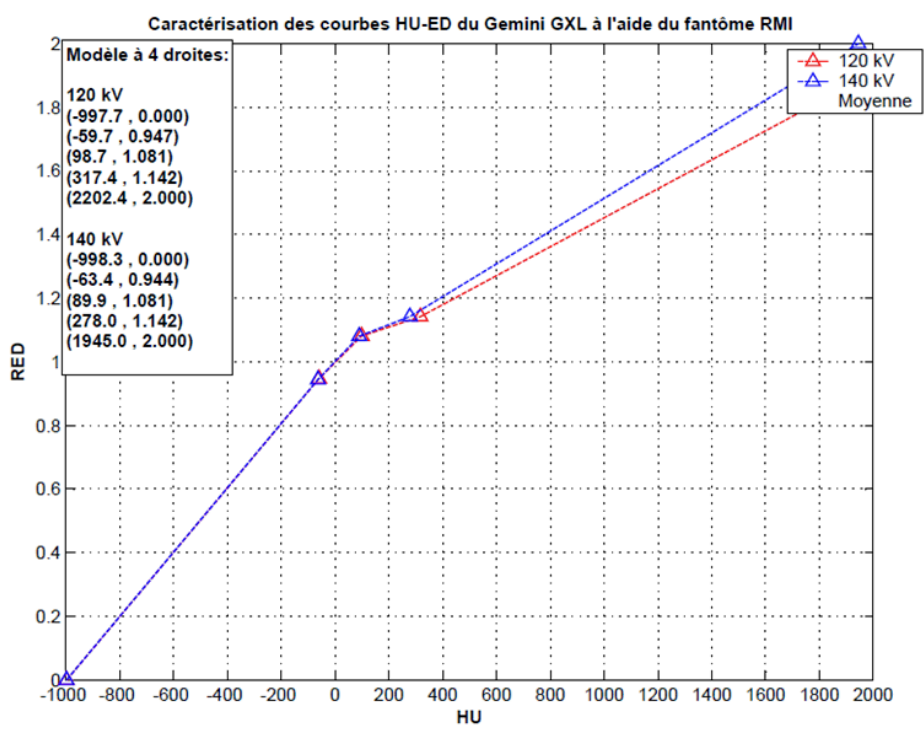

Figure 1-5: Illustrative example of an HU-ED curve. Courtesy of Centre hospitalier de l'Université de Montréal.

effective atomic numbers and the electron densities based on dual-source CT images. Application to heavy ion therapy (e.g. proton) includes the relationship between the effective atomic number and the mean ionization energy of an absorber. The limits of our definition, in addition to a sensitivity study of the extracted parameters, are assessed. A novel theoretical definition of the effective atomic number is also proposed. Chapter 2 covers a review of the photon-matter interactions as well as a description of the components of a CT scanner. A dual source CT description is also provided with an overview of the theory behind this technology and some applications. In chapter 3, a review of the literature is proposed as an introduction of the scientific paper "A stoichiometric calibration method for Dual Energy Computed Tomography " presented in the following chapter. The last chapter concludes my master's project and states the future work to be undertaken in this field of research. 


\section{Chapter 2 \\ Theory}

\subsection{X-ray interactions with matter}

When interacting with an absorber, photons follow the standard law of exponential attenuation [32] defined as

$$
I(x)=I_{0} e^{-\mu x}
$$

where $I_{0}$ is the initial beam intensity, $x$ is the thickness of the absorber and $\mu$ is the linear attenuation coefficient of the absorber in $\mathrm{cm}^{-1}$. The mass attenuation coefficient, in $\mathrm{cm}^{2} / \mathrm{g}$, is written such [32]

$$
\frac{\mu}{\rho}=\frac{\tau}{\rho}+\frac{\sigma_{R}}{\rho}+\frac{\sigma_{C}}{\rho}+\frac{\kappa}{\rho}
$$

and is composed of the photoelectric absorption $\tau$, the coherent scattering $\sigma_{R}$, the incoherent scatter $\sigma_{C}$, and the pair and triplet production $\kappa$. Depending on the atomic number of the absorber and the energy of the photon, the predominance of the interaction will differ. Figure 2-1 shows the mass attenuation coefficients for water and iron as a function of the incident photon energy. Noticeable differences between these two media are explained by figure 2-2. It represents the predominance of photoeffect for low photon energies, Compton effect for intermediate and pair production for high photon energies. For instance, water will have a predominance of Compton effect for almost all energy range $(20 \mathrm{keV}-10 \mathrm{MeV})$ [33], while iron will 
be more affected by photoelectric effect and pair production. The lines of the figure represent the equal contribution of the atomic cross section of both phenomena.

\subsubsection{Coherent scattering}

Coherent scattering, also called Rayleigh scattering, is the interaction of a photon with an atom as a whole, where the photon scatters on bound atomic electrons. The outgoing x-ray photon has essentially the same initial energy but changes in direction with a very small angle. There is no excitation and no charged particle released during the phenomenon. Therefore, there is no transfer of energy and this interaction will only increase the attenuation. The scattering angle can be estimated by the following equation [32]

$$
\theta_{R} \approx 2 \arcsin \left(\frac{0.026 Z^{1 / 3}}{\epsilon}\right),
$$

where $Z$ is the atomic number of the absorber and $\epsilon=h \nu /\left(m_{e} c^{2}\right)$. The Rayleigh mass attenuation coefficient is defined as

$$
\frac{\sigma_{R}}{\rho}=\frac{\mathrm{N}_{\mathrm{A}}}{A}{ } \sigma_{R},
$$

where ${ }_{a} \sigma_{R}$ is the atomic cross section of the coherent scattering. Because ${ }_{a} \sigma_{R} \propto Z^{2} /(h \nu)^{2}$ and $A \approx 2 Z$ for the majority of the elements, one can conclude

$$
\frac{\sigma_{R}}{\rho} \propto \frac{Z^{2}}{h \nu} .
$$

\subsubsection{Photoelectric effect}

The photoelectric effect is the interaction of a photon with a tightly bound orbital electron of an absorber atom. The photon is absorbed and an electron, called 


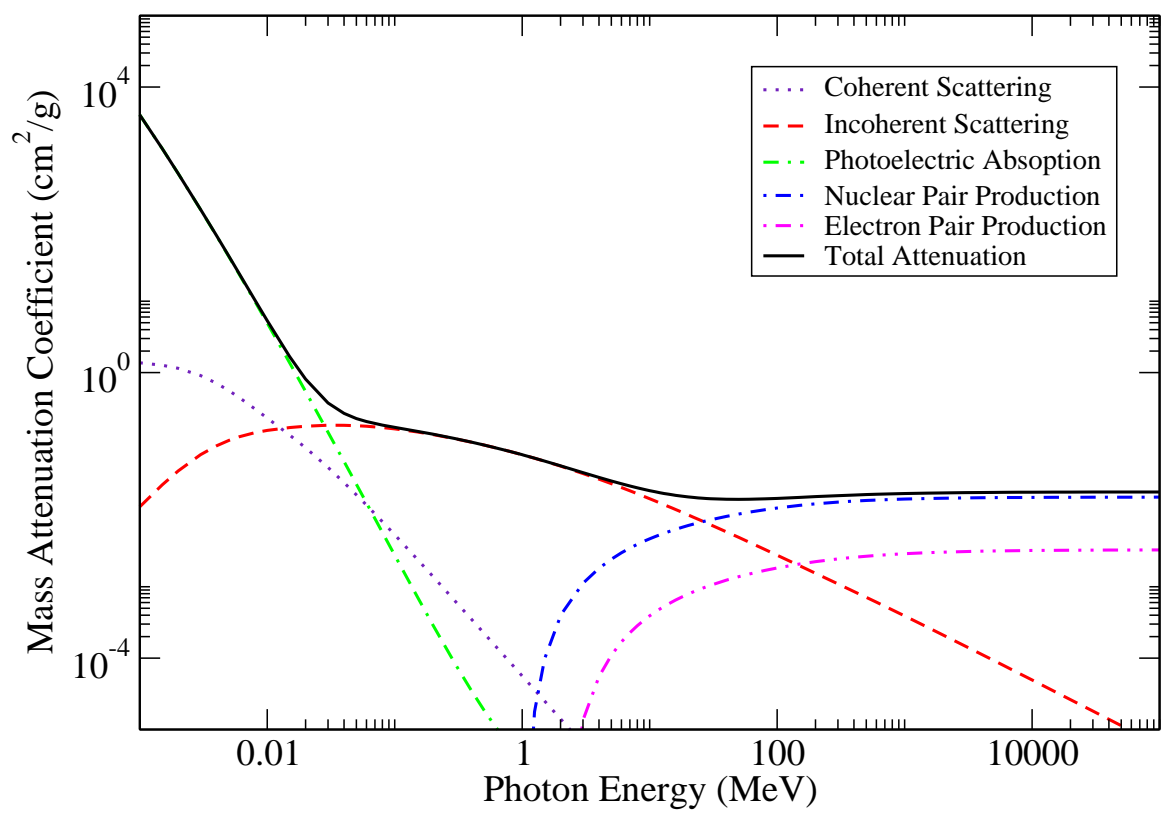

(a) Water

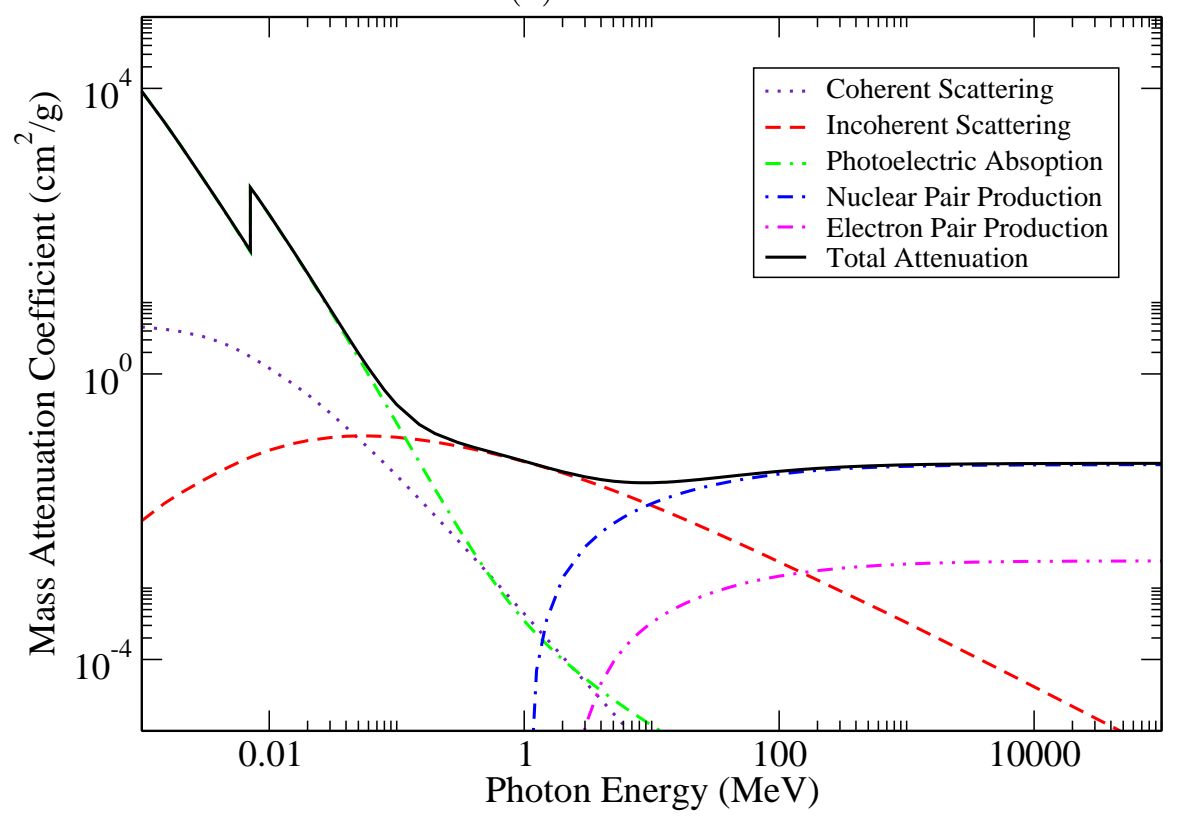

(b) Iron

Figure 2-1: Mass attenuation coefficient of water (a) and iron (b) as a function of the photon energy. Individual mass attenuation coefficients of equation 2.2 are also illustrated. Calculated from the XCOM database [6]. 


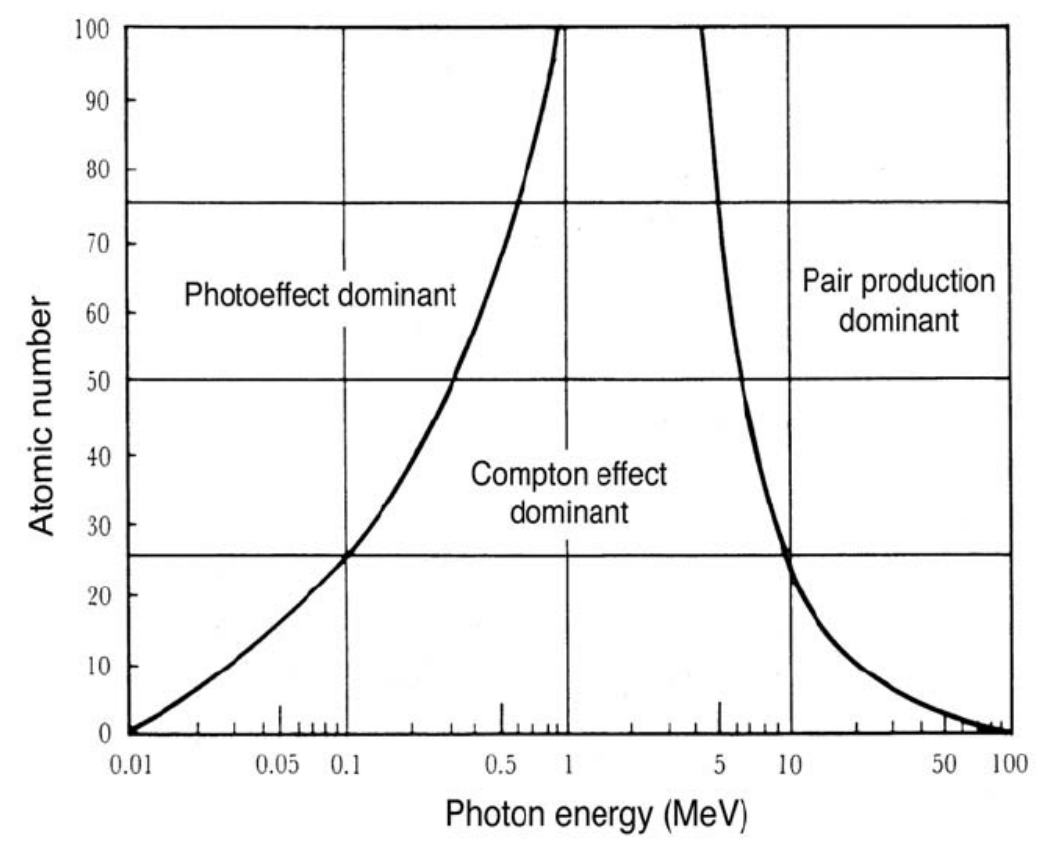

Figure 2-2: Region of relative predominance of the three main types of interactions of photons with matter [32].

photoelectron, is ejected with a kinetic energy

$$
\mathrm{E}_{K}=h \nu-\mathrm{E}_{B}
$$

where $E_{B}$ is the binding energy of the electron and $h \nu$ the energy of the incoming photon. The probability of the effect to take place is energy dependent. Indeed, the effect can only occur if the energy of the incident photon is higher than the binding energy of the orbital electron. If the incident photon has an energy close to the binding energy, the probability is large. However, if these two energies are equal, the probability drops and this produces the absorption edge noticeable on figure $2-1 \mathrm{~b}$ around $70 \mathrm{keV}$. For photons of energy larger than the binding energy of the K-shell, $\mathrm{E}_{B}(K)$, about $80 \%$ of all photoelectric interactions will occur with electrons from 

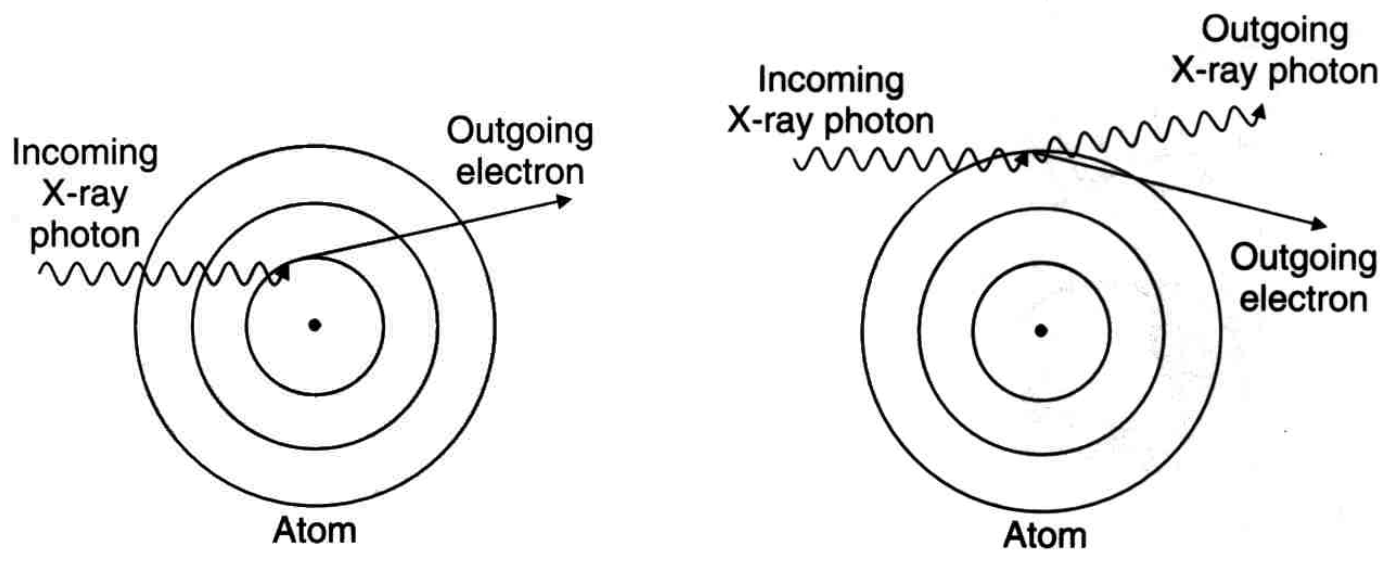

Figure 2-3: Illustrative example of the photoelectric effect (left) and the Compton scattering (right). Courtesy of http://www.physics.hku.hk/.

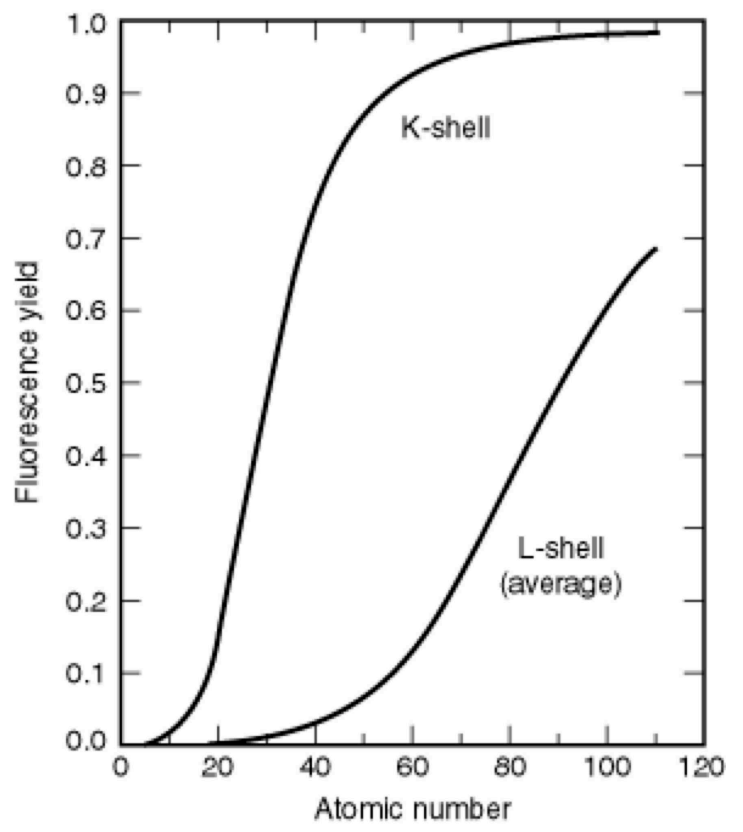

Figure 2-4: Fluorescence yield. Courtesy of http://xdb.lbl.gov/. 
that specific shell, while $20 \%$ will occur with electrons from outer shells. Once the atom is ionized, the vacancy created will be filled by an electron of a higher shell and the difference in binding energies will be emitted by either an Auger electron or a characteristic photon. The fluorescence yield, shown on figure $2-4$, is the probability for the characteristic photon emission to take place as opposed to Auger electron. This probability varies depending on the atomic shell and the atomic number. The photoelectric mass attenuation coefficient is defined as [32]

$$
\frac{\tau}{\rho}=\frac{\mathrm{N}_{\mathrm{A}}}{A}{ } \tau \propto \frac{Z^{3}}{(h \nu)^{3}},
$$

where ${ }_{a} \tau$ is the atomic cross section of the photoelectric effect. The energy transferred to charged particles is governed by the relationship [32]

$$
\overline{\mathrm{E}}_{\mathrm{tr}}^{\mathrm{PE}}=h \nu-\sum_{j} \mathrm{P}_{j} \omega_{j} \eta_{j} \mathrm{E}_{\mathrm{B}}(j)
$$

where $\mathrm{E}_{\mathrm{B}}$ is the binding energy of the subshell $j$ electron, $\mathrm{P}_{j}$ is the probability for the photoelectric effect, if it does occur, to occur in the $\mathrm{j}$ subshell, $\omega_{j}$ is the fluorescent yield for this subshell, and $\eta_{j}$ is defined as the fraction of the binding energy $\mathrm{E}_{\mathrm{B}}$ carried by the characteristic photon.

\subsubsection{Compton scattering}

The Compton scattering is the interaction between a photon and a "free" orbital electron $\left(h \nu>>\mathrm{E}_{\mathrm{B}}\right)$ of an absorber atom, releasing a recoil electron and a scattered photon at an angle $\theta$. The relationship between the scatter angle and the energies 
of the incident and the scattered photon is given as [32]

$$
h \nu^{\prime}=h \nu \frac{1}{1+\epsilon(1-\cos \theta)},
$$

where $\epsilon=h \nu /\left(m_{e} c^{2}\right)$. Figure 2-5 shows the relationship between the energy of the incident photon and the maximum and mean fractions of that energy being transferred to the recoil electron. The limits of the energy of the scattered photon go from a scattered photon of energy equals to the incident photon (classical Thomson scattering) to $0.255 \mathrm{MeV}$ in the case of backscatter. The Compton mass attenuation

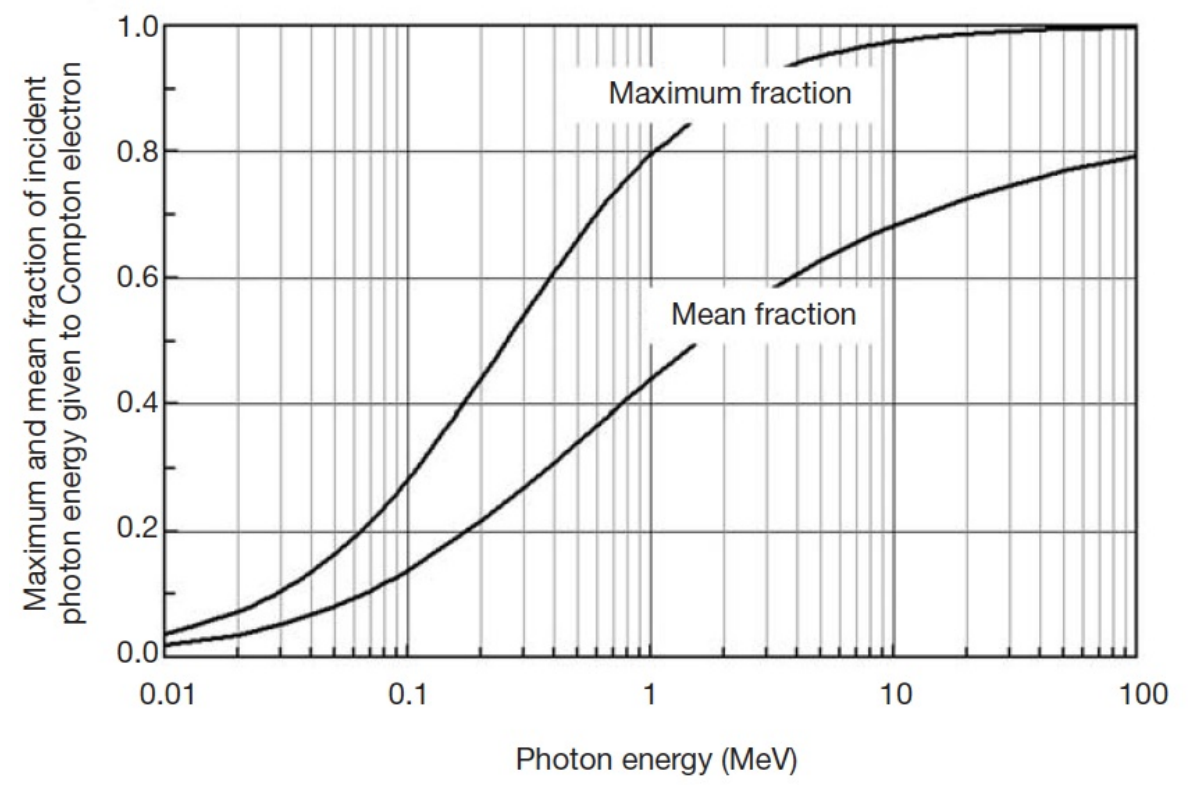

Figure 2-5: Maximum and mean fraction of the incident photon energy transferred to the recoil electron [32].

coefficient is $[32]$

$$
\frac{\sigma_{C}^{K N}}{\rho}=\frac{\mathrm{N}_{\mathrm{A}}}{A}{ }_{a} \sigma_{C}^{K N} \approx \frac{1}{2} \mathrm{~N}_{\mathrm{A}}\left({ }_{e} \sigma_{C}^{K N}\right),
$$


where ${ }_{a} \sigma_{C}^{K N}$ and ${ }_{e} \sigma_{C}^{K N}$ are the atomic and electronic cross sections of the Compton scatter respectively, making the Compton linear attenuation coefficient almost only dependent on the density of the absorber. Indeed, ${ }_{a} \sigma_{C}^{K N}=Z_{e} \sigma_{C}^{K N}$ and the $\mathrm{Z} / \mathrm{A}$ ratio is 0.4 to 0.5 for the majority of the elements in the periodic table, except for hydrogen which is approximately 1 . There is, therefore, a small but non negligible dependence on the atomic number for the mass attenuation coefficient. However, the Compton electronic cross section is independent of the atomic number. Here, the atomic and electronic cross sections are determined from the model of Klein-Nishina.

\subsubsection{Pair and triplet production}

Pair and triplet production are phenomena happening for high photon energies. In fact, the threshold for nuclear pair production is [32]

$$
E_{t h r}^{N P P}=(1.022 \mathrm{MeV}) \times\left(1+\frac{m_{e} c^{2}}{m_{\mathrm{A}} c^{2}}\right) \approx 1.022 \mathrm{MeV}
$$

while the threshold for triplet production is

$$
E_{t h r}^{T P}=4 m_{e} c^{2}=2.044 \mathrm{MeV}
$$

In the pair production process, the excess momentum of the incident photon will be absorbed by either the atomic nucleus or an orbital electron. Pair production in the field of a nucleus will result as the production of an electron and a positron. The positron will then interact with the surrounding electrons and, if at rest, two annihilation photons of about $511 \mathrm{keV}$ will be produced at almost 180 degrees as shown on figure ??. When talking of pair production in the field of an electron, the incoming photon interacts with a free orbital electron and results in two electrons and 
one positron, which will annihilated fraction of seconds after. The energy transferred to charged particles in these interactions is

$$
\bar{E}_{t r}^{P P}=h \nu-2 m_{e} c^{2}
$$

The pair production mass attenuation coefficient is calculated from

$$
\frac{\kappa}{\rho}=\frac{\mathrm{N}_{\mathrm{A}}}{A}{ }_{a} \kappa
$$

where ${ }_{a} \kappa$ is the atomic cross section of the pair and triplet production.

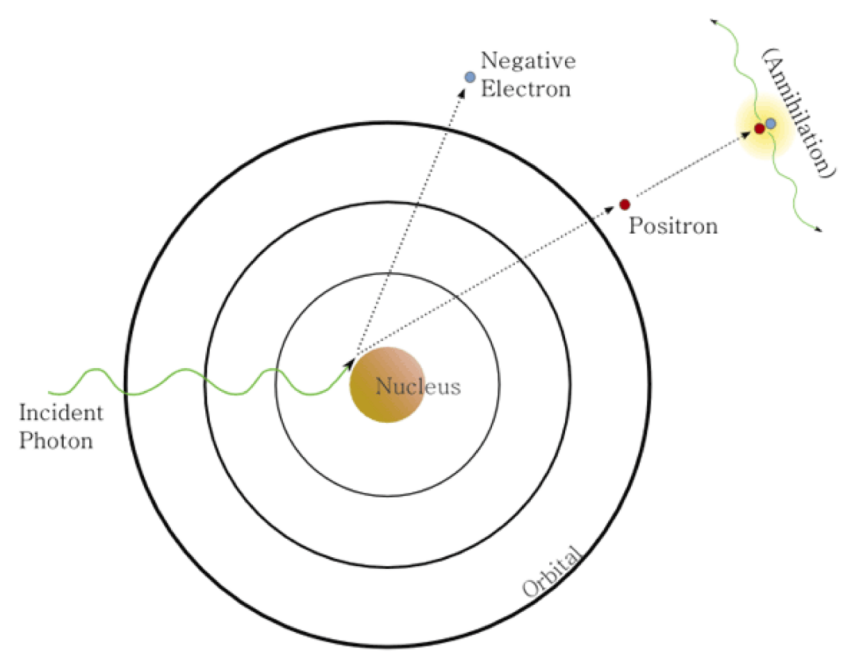

Figure 2-6: Illustrative example of the nuclear pair production. Courtesy of http://www.radiologyschools.com/.

\subsection{Computed tomography}

Many generations of CT have emerged during the past 40 years, some of them trying to solve the problems of the preceding generation. What is now called a conventional $\mathrm{CT}$ is in fact a third generation $\mathrm{CT}$ scanner but equipped with an 
helical acquisition of the patient and multiple detector array (see figure 2-7). Both the x-ray tube and the set of detectors rotate simultaneously around the subject at high speed. Because the table is moving steadily at the centre of the gantry, the path of the x-ray tube around the patient will be helical. An axial acquisition can also be performed where there is no motion of the table. A fan beam geometry is used to image a larger part of the patient at the same time. This following section explains the general operation of a CT scanner, from the production of x-rays to the reconstruction principles.

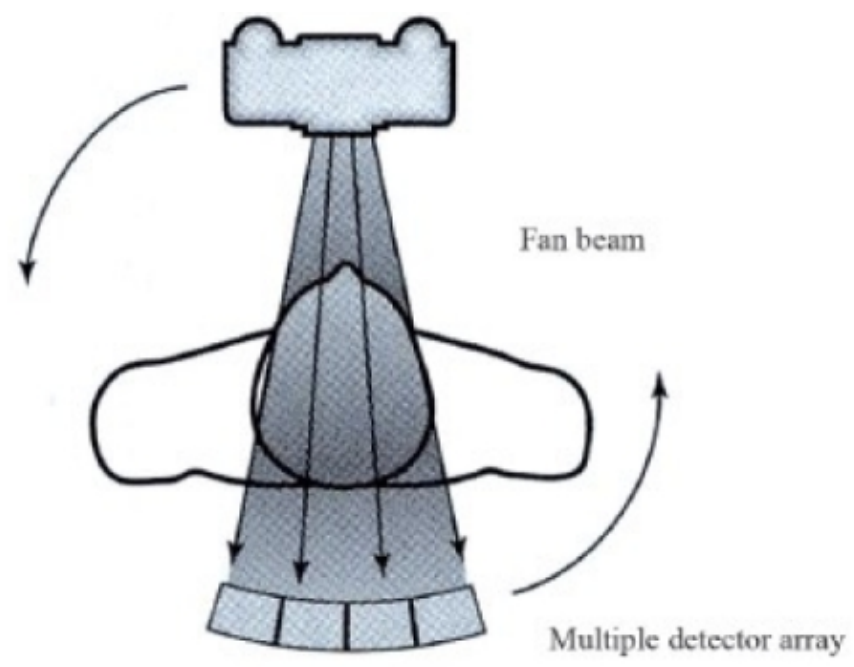

Figure 2-7: Third generation CT scanner. Courtesy of http://openi.nlm.nih.gov/.

\subsubsection{X-rays production in CT}

$\mathrm{X}$-rays are produced by an x-ray tube similar to figure $2-9$. The electrons are emitted from an heated filament by thermionic emission on the cathode side. They are then accelerated to the anode by a high potential to finally interact with the target material. The majority of the interactions results in heat deposition which is 
dissipated by the material of the anode. Bremsstrahlung radiation is emitted when

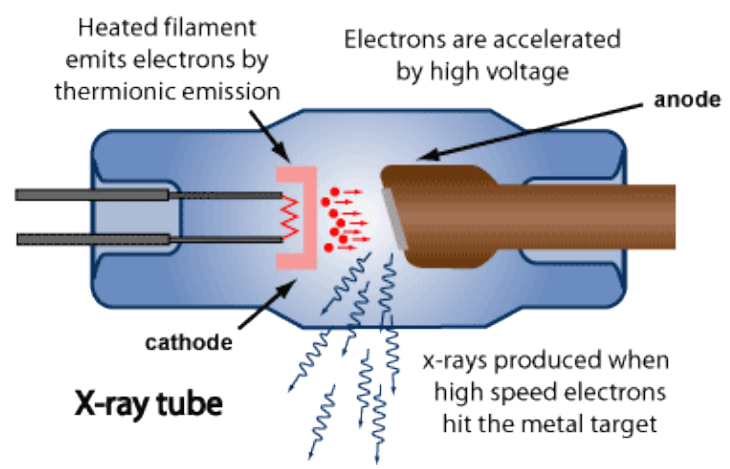

Figure 2-8: X-ray tube. Courtesy of http://www.arpansa.gov.au/.

an electron is decelerated by coulomb interactions with the nucleus of the target material and its kinetic energy is released in the form of radiation. The energy emitted by an electron will change according to the distance between itself and the nucleus and this explains why the spectrum is a continuum of energy from zero to the maximum potential, denoted $\mathrm{kVp}$, applied between the cathode and the anode. Another important type of radiation included in the spectrum is the characteristic x-rays. Indeed, some electrons may interact with the orbital electrons of the target and produce a vacancy in a shell. This vacancy can then be filled by an electron of an outer shell and the difference in binding energies of the two shells may be released in the form of radiation. Thus, the energies of the characteristic x-rays are specific to the choice of the target material. Also, the type of filtration will affect the shape of the spectrum by attenuating it.

\subsubsection{Detectors}

Solid-state detectors are used in multiple detector array CT scanners. The xrays first interact with the scintillator which is made of high density and atomic 
number materials. Visible light is released from the interactions with the scintillator and strikes the photodiode to produce an electrical signal, which is proportional to the fluence of x-rays. Also, the slice thickness is not dictated by the collimator width

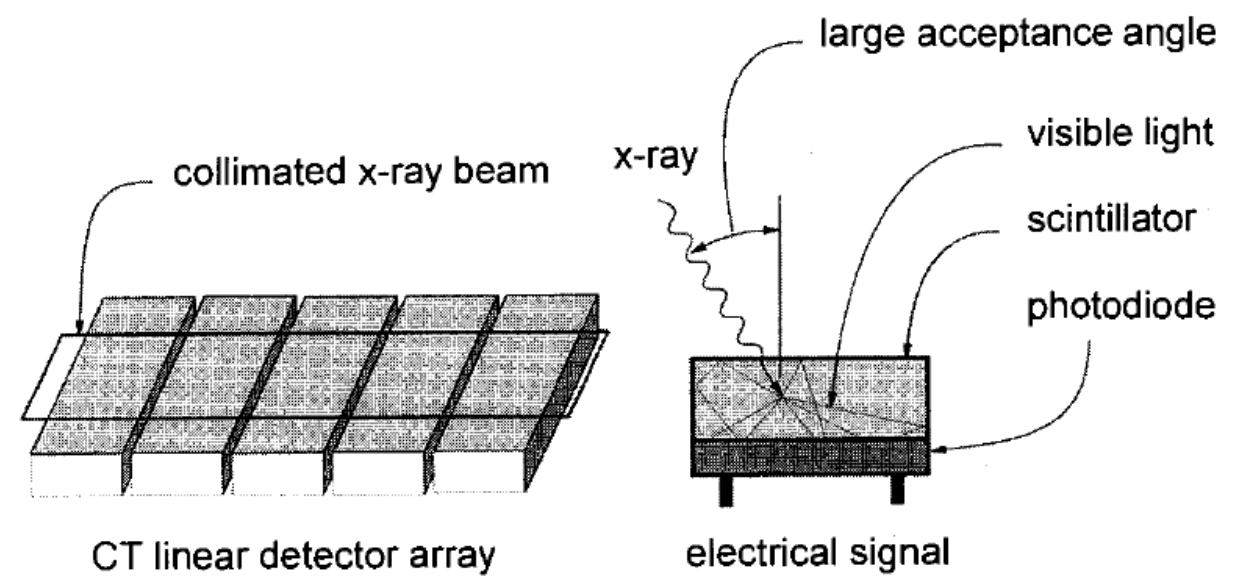

Figure 2-9: Solid-state detectors [9].

but by the detectors size. That way, a larger collimator size can be used and the signal can be averaged over many detectors to obtain an appropriate signal.

\subsubsection{Reconstruction}

The reconstruction can either be made by filtered backprojection or iterative techniques [9]. Because a majority of CT scanners uses filtered backprojection, only this reconstruction method will be discussed in this section. When imaging a subject, the detectors can only measure the intensity of the x-rays after being attenuated through the patient, which means that the total linear attenuation of a pixel of a projection is made out of the sum of all linear attenuation coefficients of the pixels along a line of response. However, from this data, one have to calculate the Hounsfield unit, or CT number, which is the unit of a pixel in a CT image and is defined as 
followed:

$$
C T(x, y)=1000 \frac{\mu(x, y)-\mu_{\text {water }}}{\mu_{\text {water }}} .
$$

From this normalization, a CT number of -1000 corresponds to air, whereas water is set to 0 and dense bone up to +3000 .

The 3-D reconstruction is obtained from a sinogram. The horizontal axis of a sinogram corresponds, for one instant in time, to the data along the line of response of a detector array and the vertical axis represents each projection angle. The filtered
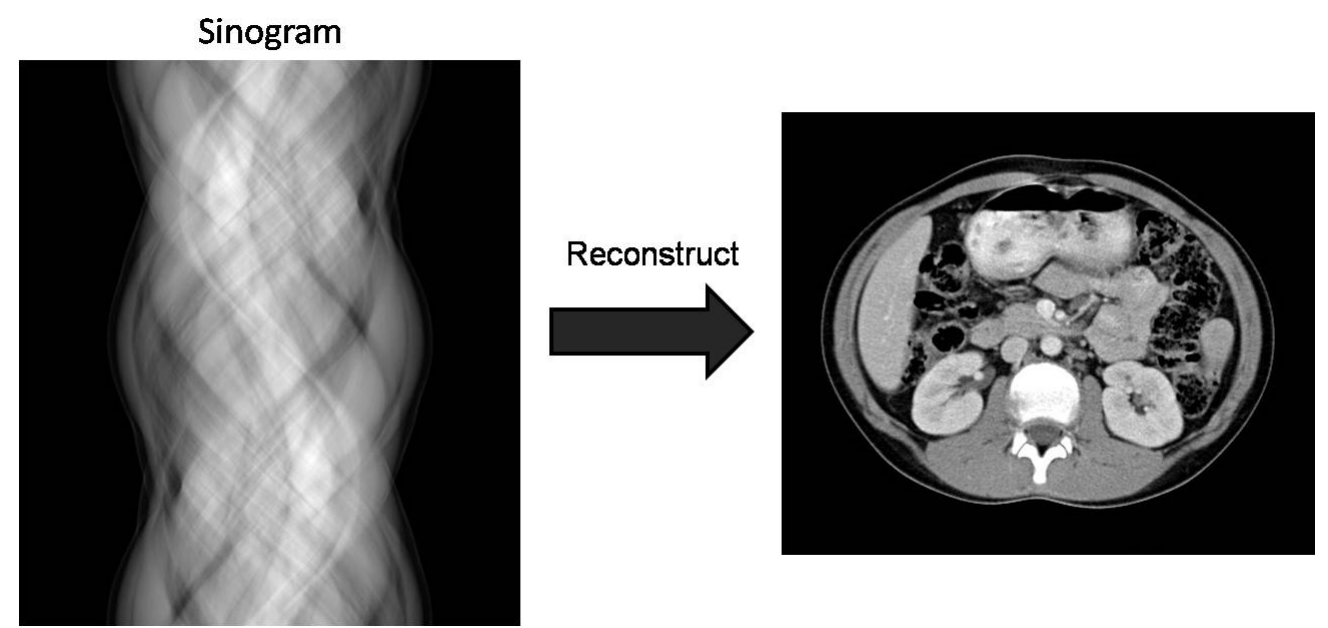

Figure 2-10: From the sinogram to the reconstruction. Courtesy of scien.stanford.edu.

backprojection reconstruction is mainly used to cancel out the $1 / \mathrm{r}$ blurring of the simple backprojection. In the frequency domain, it is written as

$$
\mathrm{p}^{\prime}(x)=\mathrm{FT}^{-1}\{\mathrm{FT}[\mathrm{p}(x)] \times \mathrm{K}(f)\}
$$

where $\mathrm{p}^{\prime}(x)$ is the backprojected data and $\mathrm{K}(f)$ is the kernel in the frequency domain. In other words, the backprojected data is obtained by taking the inverse 
Fourier transform of the multiplication of the kernel with the Fourier transform of the projection data. There are many filter types of different properties to accentuate different parts of an image. For instance, a bone filter will increase in amplitude in function of the frequency to emphasize the higher frequency details in the image, unlike a soft tissue filter that will decrease the noise but decrease the spatial resolution as well.

\subsubsection{CT artefacts}

CT reconstruction algorithm assumes a monoenergetic beam and only interactions of primary photons. Therefore, many artefacts can be produced because of scatter and beam hardening. When an object of higher atomic number or higher density is in the path, low-energy x-rays are more attenuated because of the photo-

electric effect and this will create a shift of the mean energy of the spectrum towards a higher energy. Also, scattered photons are produced as they are dominant for tissues of lower atomic number. As the preceding artefacts are physics based, other may be created by the patient itself or by the helical and multi-slice acquisition of the data. While image quality is fundamental in radiology, radiation therapy needs quantitative information on tissues rather than an empirical approach. The importance of knowing the exact $\mathrm{HU}$ for a voxel is crucial for some types of dose calculation.

\subsection{Dual Energy Computed Tomography}

The very principle of Dual Energy Computed Tomography is to image the same area with two distinct energies, monoenergetic or polyenergetic. In the specific case of Dual Source Computed Tomography, as shown in figure 2-11, two sources of different 


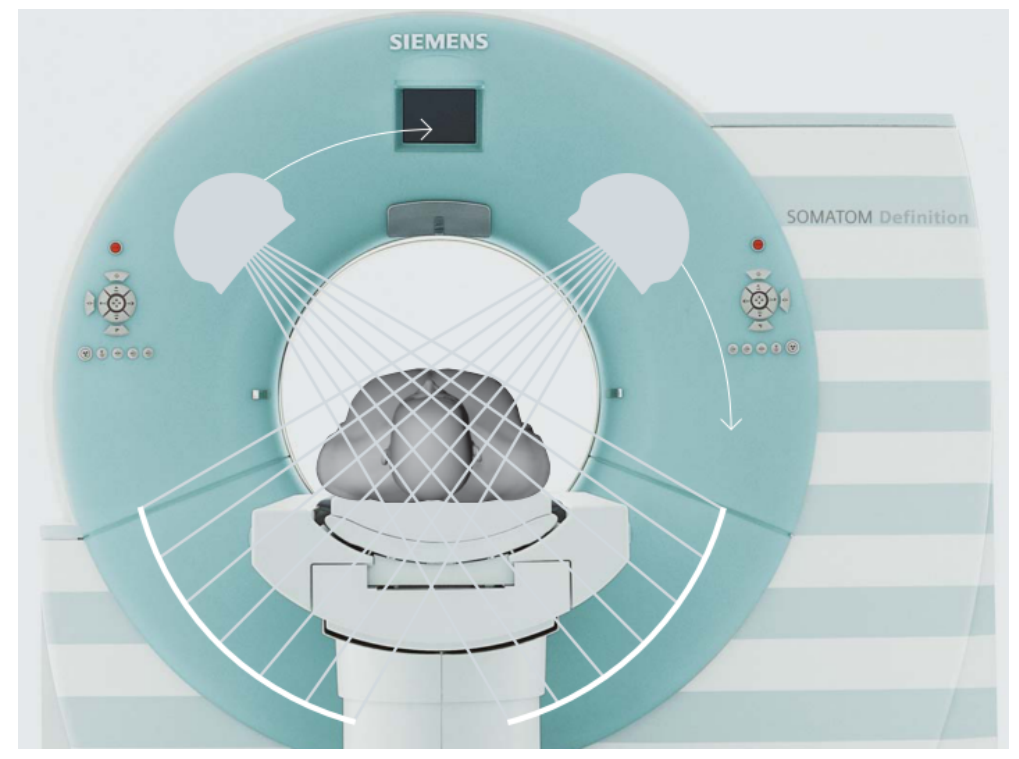

Figure 2-11: Illustration of the Siemens SOMATOM Definition Flash dual source CT.

$\mathrm{kVp}$ are positioned at about 90 degrees from one another and have their respective set of detectors. That way, both images are acquired simultaneously.

Dual Energy Computed Tomography provides not only information on the physical density of the object but also on its elemental composition. It uses the fact that images produced with two different energies will show different attenuations so that two HUs are available for the same voxel. The high dependence on the atomic number for the attenuation of low energy x-rays is mainly due to the $Z^{3}$ dependence of the photoelectric effect. However, for higher energies in the diagnostic range, the Compton effect dominates the type of photon interactions, which is almost only dependent on the physical density. An increase in attenuation can be due to either an increase of the atomic number or a higher density. 
Many DECT formalisms aim to obtain the relative electron density of a medium in addition to its effective atomic number. Thereof is defined as an atomic number, not necessarily a integer, that defines the interaction of the photons in the given medium for a range of energy.

In radiology, DECT will provide direct bone subtraction, kidney stone characterization, visualization of uric acid crystal, cartilage, ligaments, tendons and many more. In the case of radiation therapy, a more accurate dose calculation by Monte Carlo could be obtained, particularly for brachytherapy and heavy ion therapy where the electron density and the tissue elemental composition are crucial informations. 


\section{Chapter 3 \\ Introduction to a stoichiometric calibration method for dual source computed tomography}

The concept of Dual Energy CT was first introduced in a paper of 1973 by Sir Godrey Hounsfield. He stated that it was possible to enhance an area of higher atomic number within a slice by subtracting two images of $100 \mathrm{kV}$ and $140 \mathrm{kV}$, in which their normal tissue values were previously adjust to be the same [15]. The formalisms arising from the invention of $\mathrm{CT}$ are separated in different categories associated with the type of machine or radiation used : EMI scanner, single energy $\mathrm{CT}$, rapid kVp switching, synchrotron radiation and dual source $\mathrm{CT}$. For comparison purposes, the section 3.2 describes three important DECT formalisms.

\subsection{The calibration of $\mathrm{HU}$ in SECT}

The stoichiometric calibration proposed by Schneider et al. [40] is currently the most accurate to find the correspondence between Hounsfield units and electron densities. The methodology is described as follows. A phantom containing rods of known densities and elemental compositions is scanned at the energy used for treatment planning. The attenuation coefficient parametrization of Jackson and

Hawkes [19] is used and the scanner-specific constants are found with a least square fit on experimental data. The calibration is finally applied to ICRP [42] tissues. 


\subsection{Dual Energy Computed Tomography formalisms}

\subsubsection{Rutherford et al.}

In 1976, Rutherford et al. published a paper on the measurement of the effective atomic number and the electron density using an EMI scanner. He represented the linear attenuation coefficient as [36]:

$$
\begin{aligned}
\mu & =\sum f\left(Z_{i}, E\right) N_{i} \\
& =20 \times 64 E^{-3.28} \sum Z_{i}^{4.62} N_{i}+\sigma_{e}(E) \sum Z_{i} N_{i}+2 \times 80^{-2.02} \sum Z_{i}^{2.86} N_{i} .
\end{aligned}
$$

The first two terms represent respectively the photoelectric effect and the KleinNishina cross-section, whereas the last term takes account for coherence and binding energy effects. Here, $i$ is the type of atoms and $N_{i}$ is the number of atoms per unit volume. The effective number atomic number $\mathrm{Z}^{*}$ and the effective number of atoms per unit volume $\mathrm{N}^{*}$ are defined as being the values for which the real substance and the hypothetical substance should have the same absorption coefficients at all energies. Thus,

$$
\sum f\left(Z_{i}, E_{1}\right) N_{i}=f\left(Z^{*}, E_{1}\right) N^{*}
$$

and

$$
\sum f\left(Z_{i}, E_{2}\right) N_{i}=f\left(Z^{*}, E_{2}\right) N^{*} .
$$

The value of $Z^{*}$ is found by an iterative process and $N^{*}$ is obtained by substituting the value of $Z^{*}$ into the equation of either high or low energy. The information was extracted in clinic with some brain tumours in vivo by using the EMI scans at 140 $\mathrm{kVp}$ and $100 \mathrm{kVp}$. The extracted information on the electron density was accurate to about $0.5 \%$ whereas it was to about $3 \%$ for the effective atomic number. 


\subsubsection{Alvarez and Macovski}

Alvarez and Macovski [2] showed that the complete attenuation coefficient function could be represented by a linear combination of basis energy dependent functions and energy independent coefficients

$$
\mu(E)=a_{1} \frac{1}{E^{3}}+a_{2} f_{K N}(E),
$$

with $a_{1} \approx K_{1} \frac{\rho}{A} Z^{n}(n \approx 4)$ and $a_{2} \approx K_{2} \frac{\rho}{A} Z$, where $K_{1}$ and $K_{2}$ are constants, A is the atomic weight, $\rho$ is the mass density and $\mathrm{Z}$ is the atomic number. The first basis function with the dependence on $1 / E^{3}$ represents the energy dependence of the photoelectric effect. On the other hand, the second basis function is simply the Klein-Nishina function. In Computed Tomography, line integrals of the linear attenuation coefficient are measured, which is similar to measuring line integrals of the coefficients $a_{1}$ and $a_{2}$. This is shown as

$$
\int \mu(x, y ; E) d s=A_{1} \frac{1}{E^{3}}+A_{2} f_{K N}(E),
$$

where

$$
\begin{aligned}
A_{1} & =\int a_{1}(x, y) d s \\
A_{2} & =\int a_{2}(x, y) d s .
\end{aligned}
$$

Because the information of $A_{1}$ and $A_{2}$ needs to be known at every point in the projections to be able to reconstruct for $a_{1}(x, y)$ and $a_{2}(x, y)$, two different source 
spectra have to be used. That is,

$$
\begin{aligned}
& I_{1}\left(A_{1}, A_{2}\right)=T \int S_{1}(E) \exp \left[-A_{1} \frac{1}{E^{3}}-A_{2} f_{K N}(E)\right] d E \\
& I_{2}\left(A_{1}, A_{2}\right)=T \int S_{2}(E) \exp \left[-A_{1} \frac{1}{E^{3}}-A_{2} f_{K N}(E)\right] d E
\end{aligned}
$$

where $\mathrm{T}$ is the total measurement time, $S_{1}$ and $S_{2}$ are the energy spectra, and $I_{1}$ and $I_{2}$ are the total energies.

\subsubsection{Bazalova et al.}

The formalism of Bazalova et al [3] is an adaptation of the linear attenuation coefficient notation proposed by Torikoshi et al [47]. It was adapted from synchrotron radiation to polyenergetic x-ray beams to be suitable for clinical purposes. It stated that, for a material at energy E, $\mu$ can be approximated with

$$
\mu(E)=\rho_{e}^{\prime}\left(Z^{4}(F(E, Z)+G(E, Z))\right.
$$

where $\rho_{e}^{\prime}$ is the electron density of the material and $F(E, Z)$ and $G(E, Z)$ are quadratic fits of NIST attenuation coefficients for photoelectric and scattering (Rayleigh and Compton) terms. In the case of a continuous energy spectrum $j$, with spectral distribution $w_{j i}$ at energy $E_{j i}$, the equation can be written as

$$
\mu_{j}=\rho_{e}^{\prime} \sum_{i}\left[w _ { j i } \left(Z^{4}\left(F\left(E_{j i}, Z\right)+G\left(E_{j i}, Z\right)\right]\right.\right.
$$

The definition used to calculate the value of the effective atomic number is the Mayneord's method [26] described in section 3.3.1, where $n=3.5$ was chosen

$$
Z=\left(\sum_{i} w_{i} Z_{i}^{3.5}\right)^{1 / 3.5}
$$


Also, the relative electronic density is defined as

$$
\rho_{e}=\frac{\rho_{e}^{\prime}}{\left(\rho_{e}^{\prime}\right)_{w}}=\frac{N_{A} \rho \frac{Z}{A}}{N_{A} \rho_{w} \frac{Z_{w}}{A_{w}}} .
$$

Using the Hounsfield units from two different spectra $j$, one can obtain the relative attenuation coefficients of materials, as given by

$$
\frac{\mu_{j}}{\mu_{j w}}=\frac{H U_{j}}{1000}+1
$$

Introducing the linear attenuation coefficients of water in (3.10), the following equation is obtained in order to find $\mathrm{Z}$.

$$
\begin{aligned}
0= & Z^{4}-\left\{\left(\frac { \mu _ { 2 } } { \mu _ { 2 w } } \sum _ { i } w _ { 2 i } \left[Z_{w}^{4}\left[F\left(E_{2 i}, Z_{w}\right)+G\left(E_{2 i}, Z_{w}\right)\right] \sum_{i} w_{1 i} G\left(E_{1 i}, Z\right)\right.\right.\right. \\
& \left.-\frac{\mu_{1}}{\mu_{1 w}} \sum_{i} w_{1 i}\left[Z_{w}^{4}\left[F\left(E_{1 i}, Z_{w}\right)+G\left(E_{1 i}, Z_{w}\right)\right] \sum_{i} w_{2 i} G\left(E_{2 i}, Z\right)\right)\right\} \\
& \left(\frac { \mu _ { 1 } } { \mu _ { 1 w } } \sum _ { i } w _ { 1 i } \left[Z_{w}^{4}\left[F\left(E_{1 i}, Z_{w}\right)+G\left(E_{1 i}, Z_{w}\right)\right] \sum_{i} w_{2 i} F\left(E_{2 i}, Z\right)\right.\right. \\
& \left.-\frac{\mu_{2}}{\mu_{2 w}} \sum_{i} w_{2 i}\left[Z_{w}^{4}\left[F\left(E_{2 i}, Z_{w}\right)+G\left(E_{2 i}, Z_{w}\right)\right] \sum_{i} w_{1 i} F\left(E_{1 i}, Z\right)\right)\right\}
\end{aligned}
$$

According to the previous definition, the effective atomic number of water is set to 7.733. The relative electron density is found with

$$
\rho_{e}=\frac{\mu_{j}}{\mu_{j w}} \frac{\sum_{i} w_{j i}\left[Z_{w}^{4}\left[F\left(E_{j i}, Z_{w}\right)+G\left(E_{j i}, Z_{w}\right)\right]\right.}{\sum_{i} w_{j i} \cdot\left[Z^{4}\left[F\left(E_{j i}, Z\right) \cdot+G\left(E_{j i}, Z\right)\right]\right.} .
$$

The effective atomic number and the relative electron density are calculated iteratively by a MATLAB (The Mathworks, Natick, MA) routine. In the study, the 
spectral distributions $w_{j i}$ of 100 and $140 \mathrm{kVp}$ spectra were used and NIST attenuation coefficients for elements with $\mathrm{Z}$ from 5 to 15 were calculated.

While the paper was published with results from experiments done with a SECT, this formalism has now been applied to simulations, experiments with DECT as well as theoretical studies.

\subsection{Definitions of the effective atomic number}

The paper in the next chapter introduces a new definition of the effective atomic number. However, many definitions already exist in the literature. Here are the formulations of effective atomic numbers according to Mayneord [26], Manohara et al. [25] and Taylor et al. [45].

\subsubsection{Mayneord}

The formulation of the effective atomic number is

$$
Z_{\text {med }}^{n}=\sum_{i=1}^{N} w_{i} Z^{n}
$$

where $w_{i}$ is the fractional weight of $i$ the element $i=1,2, \ldots$ and $n$ is an energy dependent parameter. Note that a typical value of $n=2.94$ is used for radiology. Thus, for a certain energy range, this definition is independent of energy. Note that Bazalova et al. used $n=3.5$.

\subsubsection{Manohara et al.}

The definition of Manohara et al. is given by

$$
Z_{\text {med }}^{*} \equiv \frac{{ }_{a} \sigma_{\text {med }}(E)}{{ }_{e} \sigma_{\text {med }}(E)}
$$

which is simply the ratio of the atomic and the electron cross sections. 


\subsubsection{Taylor et al.}

For a given energy E, there exists a $Z_{\text {med }}$ such that

$$
{ }_{a} \sigma_{\text {med }}(E)={ }_{a} \hat{\sigma}\left(E, Z_{\text {med }}\right)
$$

where ${ }_{a} \hat{\sigma}$ is a fit of the atomic cross-section as a function of $Z$. Note that ${ }_{a} \hat{\sigma}(E, Z)=$ ${ }_{a} \sigma(E, Z)$ for $Z$ an integer. In the case of an energy spectrum, $\Psi(E)$, the effective atomic number is evaluated as

$$
Z_{\mathrm{med}}=\sum_{i} Z_{\mathrm{med}}\left(E_{i}\right) \Psi\left(E_{i}\right) \cong \int_{E_{\min }}^{E_{\max }} Z_{\mathrm{med}}(E) \Psi(E) d E
$$

\subsubsection{The novel definition}

We propose the following definition. For a given energy $E$, there exists a $Z_{\text {med }}$ such that

$$
e^{\sigma_{m e d}}=e^{\hat{\sigma}\left(Z_{m e d}\right)}
$$

where $e_{e} \hat{\sigma}$ is a fit of the electronic cross-section as a function of $Z$. Note that $e_{e} \hat{\sigma}(Z)=$ ${ }_{e} \sigma(Z)$ if $Z$ an integer, i.e, $Z=1,2, \ldots$. For a polyenergetic beam, there exists a $Z_{\text {med }}$ such that

$$
e^{\bar{\sigma}_{m e d}}=f\left(Z_{\text {med }}\right)
$$

where $f\left(Z_{\text {med }}\right)$ is a fit of the average electronic cross sections over all the energies of the spectrum as a function of $Z$.

\subsection{Objectives, results and impact of the scientific paper}

As seen in the previous sections of this chapter, many DECT formalisms are dependent on the spectra of the two sources. This information is not easily available 
and usually demands a none-disclosure agreement (NDA) with the manufacturer of the CT. The spectra provided might also be different than the actual spectra depending on the type of filters used during the imaging process. Another important point is the evolution of the spectrum through the patient. Indeed, because of the interactions with higher atomic number or densities, the hardening of the spectrum will happen and a question arises from this problem : Which spectrum should be taken for calculations? The initial spectrum of the manufacturer, the spectrum filtered by half the thickness of the patient, or the spectrum filtered by the entire thickness of the patient?

The main idea behind the paper presented in the next chapter is to propose a formalism easily implementable to the clinic environment in order to extract parameters from two DECT images. This study presents a simple and accurate way, achievable by the calibration of a phantom of known composition of its tissue substitutes. In other words, the stoichiometric calibration of Schneider et al. is imported to DECT with an extended parametrization of the attenuation coefficient. In this formalism, the only need for spectrum information is in the definition of the effective atomic number. As seen in section 3.3, this one differs from the other definitions since it takes into account the electronic cross section of the media for the difference in photon-matter interactions depending on the energy of the photon. However, the variation of the effective atomic number for energies in the diagnostic range is shown to be almost negligible which allows the use of an average effective atomic number for all tissues of interest. 
The majority of actual treatment planning systems, like Eclipse with the anisotropic analytical algorithm (AAA), perform calculations of dose distributions by assuming water equivalent phantoms and a variation in electron densities when inhomogeneity corrections are enabled. The impact of finding accurate parameters would be to calculate with Monte Carlo techniques more realistic dose distributions that would consider both the electron density and the effective atomic number. The maps of both quantities (see figure 3-1) could be directly implemented in the treatment planning system and, consequently, HU-ED curves would become obsolete. Also, for dose distributions in protons or heavy ions therapy, HU-SP curves are currently used which relates the $\mathrm{HU}$ to the particle stopping power. By finding a relationship between the effective atomic number and the mean excitation energy, combining to the map of electron density, it is possible to directly produce a map of the particle stopping power.

\subsection{Contribution of authors}

\section{Alexandra Bourque}

- General discussion and ideas of the project

- Experimental measurements

- Computational implementation of the formalism

- Calculation and result analysis

- Redaction and revision of the paper

Jean-Francois Carrier

- General discussion and ideas of the project

- Redaction and revision of the paper 


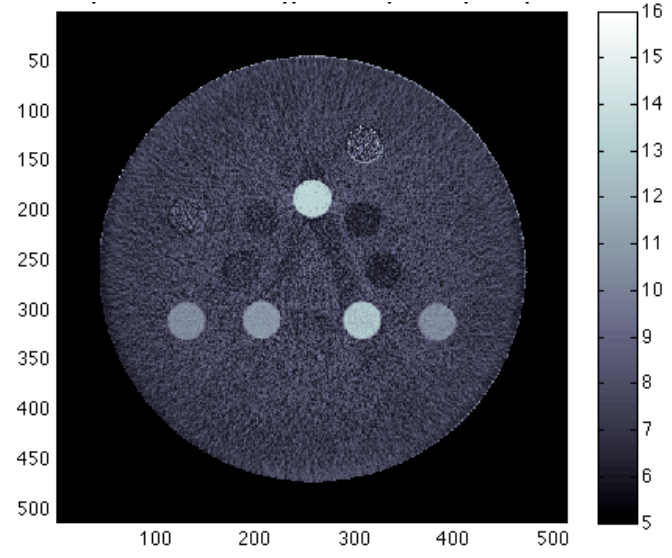

(a) Effective atomic number

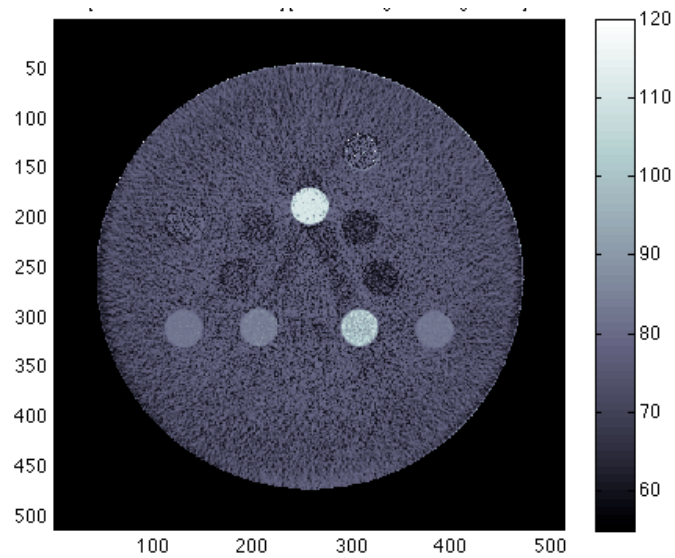

(c) Mean excitation energy

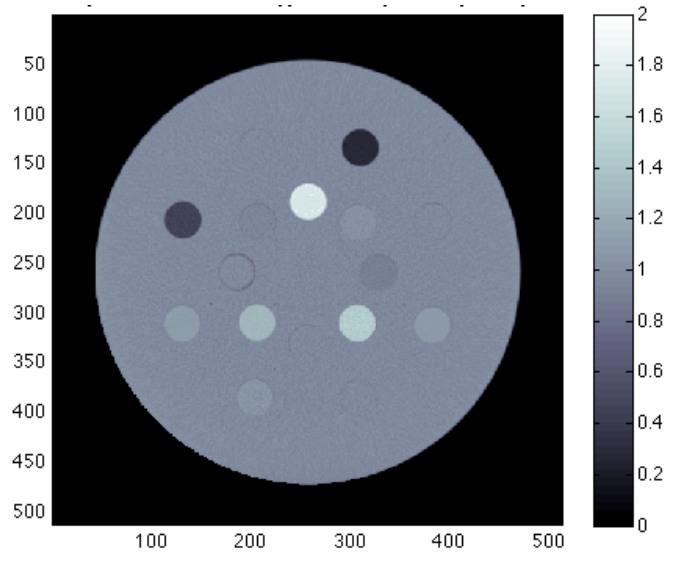

(b) Relative electron density

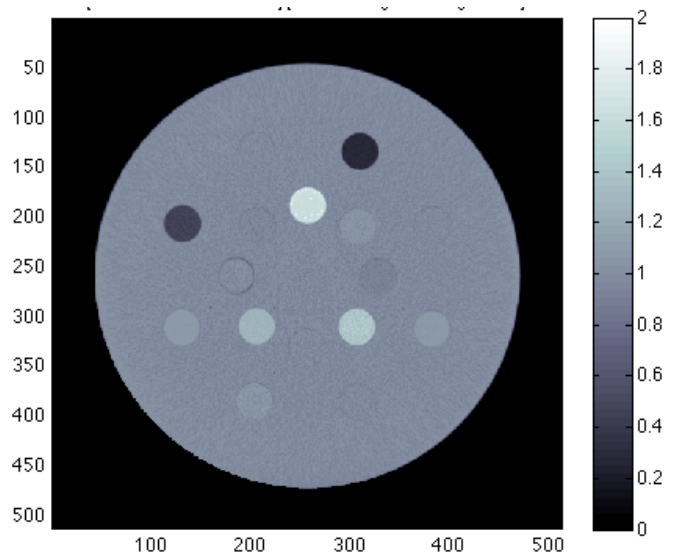

(d) Relative proton stopping power

Figure 3-1: Maps of the extracted parameters for the Gammex-467 phantom. Note that the map of the relative proton stopping power is computed for a proton of kinetic energy equals to $216 \mathrm{MeV}$. 
Hugo Bouchard

- Supervision and direction of the project

- Methodology and mathematical formalism

- Redaction and revision of the paper

- Enhancement of the paper's scientific impact

\subsection{Permissions}

The paper "A stoichiometric calibration method for dual source computed tomography" has been submitted for publication. 


\title{
Chapter 4
}

\section{A stoichiometric calibration method for dual source computed tomography}

\author{
Alexandra E. Bourque \\ Medical Physics Unit, Montreal General Hospital (L5-113), McGill University, 1650 Cedar \\ Avenue, Montreal, Quebec H3G 1A4, Canada \\ Centre hospitalier de l'Université de Montréal (CHUM), 1560 Sherbrooke est, Montréal, \\ Québec H2L 4M1, Canada

\section{Jean-François Carrier} \\ Centre hospitalier de l'Université de Montréal (CHUM), 1560 Sherbrooke est, Montréal, \\ Québec H2L 4M1, Canada \\ Département de physique, Université de Montréal, Pavillon Roger-Gaudry (D-428), 2900 \\ Boulevard Édouard-Montpetit, Montréal, Québec H3T 1J4, Canada
}

\section{Hugo Bouchard}

Centre hospitalier de l'Université de Montréal (CHUM), 1560 Sherbrooke est, Montréal, Québec H2L 4M1, Canada

Département de physique, Université de Montréal, Pavillon Roger-Gaudry (D-428), 2900 Boulevard Édouard-Montpetit, Montréal, Québec H3T 1J4, Canada 


\section{ABSTRACT}

The accuracy of radiotherapy dose calculation relies crucially on patient composition data. The CT calibration methods based on the stoichiometric calibration of Schneider et al. (1996) are the most reliable to determine electron density (ED) with commercial single energy CT (SECT) scanners. Along with the recent developments in dual energy CT (DECT) commercial scanners, several methods were published to determine $\mathrm{ED}$ and the effective atomic number (EAN) for polyenergetic beams without the need for CT calibration curves. This paper intends to show that with a rigorous definition of the EAN and an improved cross section parametrization, the stoichiometric calibration method can be successfully extended to DECT with significant accuracy improvements with respect to literature without the need for spectrum measurements or empirical beam hardening corrections. Using a theoretical framework of ICRP compositions and the XCOM cross section database, the revised stoichiometric calibration formalism yields HU predictions within less than $\pm 1 \mathrm{HU}$ of the theoretical $\mathrm{HU}$ calculated from XCOM data averaged over the spectra used (e.g., $80 \mathrm{kVp}, 100 \mathrm{kVp}, 140 \mathrm{kVp}$, and 140/Sn kVp). A fit of mean excitation energy ( $I$-value) data as a function of EAN is provided in order to determine the heavy-ion stopping power of ICRP tissues from ED-EAN measurements. Analysis of the calibration phantom measurements with the Siemens SOMATOM Definition Flash dual source CT scanner shows that the present formalism yields mean absolute errors of $(0.3 \pm 0.4) \%$ and $(1.6 \pm 2.0) \%$ on ED and EAN respectively. For heavyion therapy, the mean absolute errors for calibrated $I$-values and proton stopping powers $(216 \mathrm{MeV})$ are $(4.1 \pm 2.7) \%$ and $(0.5 \pm 0.4) \%$ respectively. In all clinical 
situations studied, the uncertainty on heavy-ion ranges in water for therapeutic energies are found to be less than $1.3 \mathrm{~mm}, 0.7 \mathrm{~mm}$ and $0.6 \mathrm{~mm}$ for protons, helium and carbon ions, respectively using a generic reconstruction algorithm (FBP). With a more advanced method (SAFIRE iterative technique), the values become $1.0 \mathrm{~mm}$, $0.5 \mathrm{~mm}$ and $0.4 \mathrm{~mm}$ for protons, helium and carbon ions, respectively. These results allow to conclude that the present extension of the stoichiometric calibration yields a highly-accurate method for characterizing tissue with DECT.

Keywords : Tissue Characterization, Spectral Imaging, Computed Tomography, Dual Energy, Electron Density, Stopping Power, Mean Excitation Energy, Effective Atomic Number

\subsection{Introduction}

In radiotherapy, Computed Tomography (CT) imaging is the gold standard method to obtain patient-specific data for treatment planning. To account for tissue heterogeneities, dose calculation engines require $\mathrm{CT}$ calibration curves which link, in each voxel, the Hounsfield unit (HU) to a physical characteristic such as the electron density (ED) or property like stopping power (SP). When it comes to determining ED or SP maps using conventional CT scanners, methods based on the stoichiometric calibration proposed by Schneider et al. [40] yield more reliable results than the

use of direct phantom-based CT calibrations [40, 41, 14, 49]. For analytical types of dose calculation algorithms, such as photon convolution/superposition methods [1] or proton range calculations [38], HU-ED or HU-SP curves have sufficient information 
to perform these calculations. However, when using transport-based algorithms such as Monte Carlo techniques, the elemental compositions need to be known to establish material cross sections $[11,30]$.

Common tissue characterization methods using CT calibration curves assume that material compositions are discretizable into specific ranges of $\mathrm{HU}$ where either a fixed composition is used [12, 20] or a mixture of two tissues is assumed [41]. Although such methods can yield reasonable accuracy in determining ED (e.g., ref. [29]), uncertainty on HU measurements can cause tissue misallocation and yield errors on absorbed dose calculation of the order of $10 \%$ or more [5]. Moreover, while the non-bijectivity of the HU-ED (or HU-SP) relation requires performing multi-linear fits, problems arise when it comes to defining the number of segments and their general trend $[46,50]$. It is therefore recognized that SECT calibration curves are limitating when it comes to rigorously characterizing tissue for treatment planning.

In a recent study, Yang et al. [52] theoretically showed the superiority of the DECT method against standard clinical practice for determining material properties relevant to dose calculations. While early developments in CT imaging proposed the use of dual-energy CT (DECT) to extract additional information [15, 2, 36], recent technological developments in spectral CT [39], dual-energy CT[10] and dual source CT (DSCT) $[13,31]$ are expected to improve tissue characterization methods for radiotherapy. To characterize tissue properties from DECT images, several approaches were recently proposed in the literature. An approach dealing with monoenergetic synchrotron radiation was proposed by Torikoshi et al. [47], allowing to extract ED 
and the effective atomic number (EAN), yielding an overall accuracy around $\pm 1 \%$ when determining ED experimentally [48]. Another formalism was also developed for monoenergetic synchrotron radiation the same year by Kirby et al. [22] allowing to predict ED measurements with an accuracy of about $5 \%$. The formalism of Torikoshi et al. [47] was further adapted by Bazalova et al. [3] for commercial scanners which use polyenergetic photon spectra yielding an accuracy of $1.8 \%$ on average for $\mathrm{ED}$ and $2.8 \%$ on average for EAN, this using phantom measurements. The same formalism was used by Landry et al. [23] to compare experiments with Monte Carlo simulations of a CT scanner, yielding an agreement within $\pm 5 \%$ both for ED and EAN. Another formalism was published by Mahnken et al. [24] and demonstrated the ability to distinguish body fluids with a precision of about $0.02 \mathrm{~g} / \mathrm{cm}^{3}$ and 0.1 in determining mass density and EAN respectively. A formalism was also proposed by Midgley [28] which parameterized the attenuation coefficient as a function of ED and a compositional ratio (i.e., $R_{4}$, being a function of the EAN to the fourth power) yielding a theoretical accuracy of about 1-2\% for ED. Finally, the method proposed by Saito [37] to establish a relation between $\Delta \mathrm{HU}$ and ED theoretically demonstrated an agreement within $0.7 \%$ and $2.5 \%$ for simulated and measured ED respectively.

The robustness of these mathematical formalisms to be used with commercial CT scanners relies on the accuracy to which cross sections are parameterized as a function of physical parameters as well as the knowledge of the imaging photon spectrum. At the basis of the stoichiometric calibration of Schneider et al. (1996), the parametrization of Jackson and Hawkes [19] relies on Mayneord's power law [26, 44] and is limited when it comes to modelling the photoelectric effect in human tissues 
containing high- $Z$ materials, such as bone [51] or the thyroid [52]. Other problems also arise with the attempt to consistently defining the EAN using a power law, as the choice of the exponent is arbitrary [3, 23, 37]. One advantage in using an extended cross section parametrization $[47,22,27]$ is to improve the accuracy to which one can model high- $Z$ components. As for the knowledge of the photon spectrum, the method of Bazalova et al. $[3,4,23]$ is based on experimental spectrum measurements and empirical beam hardening corrections, while the method of Mahnken et al. [24] assumes a predetermined spectrum and neglects beam hardening effects. In both methods, either experimental measurements or reliable information from the manufacturer is required.

The main idea of the present study is to implement the stoichiometric calibration method of Schneider et al. (1996) in DECT with the goal of making it suitable for commercial CT scanners. The theory is constructed as follows. The XCOM cross section database [6] is parameterized using a polynomial expansion [22, 27], yielding more degrees of freedom than the model of Jackson and Hawkes (1981). Providing a rigorous definition of the EAN, the relation between $\mathrm{HU}, \mathrm{ED}$ and EAN is theoretically established and expressible using linear matrix systems. The equation system is generalized for DECT, providing solutions for ED and EAN based on HU pairs. Using a XCOM-based theoretical framework, the developed mathematical formalism is thoroughly validated. A solution for the determination of the mean excitation energy (the $I$-value) from EAN is provided, making the method applicable to proton and heavy-ion therapy. Experimental measurements using a Siemens SOMATOM Definition Flash DSCT are performed to validate the method for clinical use. A 
thorough uncertainty analysis allows determining the robustness of the method and compares it to other methods in the literature.

\subsection{Theory}

\subsubsection{The SECT stoichiometric calibration of Schneider et al.}

This section summarizes the single-energy CT (SECT) stoichiometric calibration proposed by Schneider et al. (1996). Note that the following description is made in the scope of the present paper and therefore a few differences are present, although the main idea of Schneider et al. (1996) is respected. For a given energy, the attenuation coefficient (in $\mathrm{cm}^{-1}$ ) is modelled by the parameterization of Jackson and Hawkes (1981) as follows:

$$
\mu=n_{e}\left\{k_{p h} \tilde{Z}^{3.62}+k_{c o h} \hat{Z}^{1.86}+k_{K N}\right\}
$$

where $k_{p h}, k_{c o h}$ and $k_{K N}$ are constants characterizing their respective cross section. Note that $k_{K N}$ is directly the Klein-Nishina cross section. The electronic density $n_{e}$ (in $\mathrm{el} / \mathrm{cm}^{3}$ ) is given by

$$
n_{e}=\rho N_{\mathrm{A}} \frac{Z}{A}
$$

where $\rho$ is the mass density (in $\mathrm{g} / \mathrm{cm}^{3}$ ), $N_{\mathrm{A}}$ is Avogadro's number (in at $/ \mathrm{mol}$ ), $Z$ is the atomic number (in el/at) and $A$ is the molar mass (in $\mathrm{g} / \mathrm{mol}$ ).

The values of $\tilde{Z}$ and $\hat{Z}$ are calculated with the known chemical composition of each material (or mixture) with the following power law additivity rule [26, 44]

$$
Z_{m}=\left[\sum_{i} \lambda_{i} Z_{i}^{m}\right]^{1 / m}
$$


such that $\tilde{Z} \equiv Z_{3.62}$ and $\hat{Z} \equiv Z_{1.86}$. Here $\lambda_{i}$ is the fraction of electrons from the $i^{\text {th }}$ element in the mixture given by

$$
\lambda_{i}=\frac{\frac{\omega_{i} Z_{i}}{A_{i}}}{\left(\frac{Z}{A}\right)_{\text {med }}}
$$

with the mean number of electron per atomic mass given by

$$
\left(\frac{Z}{A}\right)_{\mathrm{med}}=\sum_{i} \omega_{i} \frac{Z_{i}}{A_{i}}
$$

and $w_{i}$ the fractional of the $i^{\text {th }}$ element.

From equation 4.1, the linear attenuation coefficient of each material relative to water can be expressed as

$$
u \equiv \frac{\mu}{\mu_{\mathrm{w}}}=\rho_{e}\left\{k_{p h}^{*} \tilde{Z}^{3.62}+k_{c o h}^{*} \hat{Z}^{1.86}+k_{K N}^{*}\right\} .
$$

with $\rho_{e}$ the electron density of the medium relative to water. The constants $k_{p h}^{*}, k_{c o h}^{*}$ and $k_{K N}^{*}$ include the normalization to water. It is convenient to define the relative attenuation coefficient $u$ as reduced Hounsfield Unit (or reduced HU), since it can be obtained from $\mathrm{HU}$ as follows

$$
u \equiv \frac{\mathrm{HU}+1000}{1000} .
$$

Note that this definition implies that the $\mathrm{HU}$ of water is 0 , while Schneider et al. (1996) defined the HU of water to be 1000. In order to find the values of $k_{p h}^{*}, k_{c o h}^{*}$ and $k_{K N}^{*}$ using a least square fit on experimental data measured with a calibration phantom (and known densities and elemental compositions). Once these scanner-specific constants are known for each energy (or even for each scanning protocol), a calibration with human tissues is performed by computing the theoretical values of $\mathrm{HU}$ 
with equation 4.6 using elemental compositions of human tissues recommended by the International Commission on Radiological Protection (ICRP) [42]. The relative electron density is calculated with

$$
\rho_{e} \equiv \frac{n_{e, \mathrm{med}}}{n_{e, \mathrm{w}}}=\rho_{\mathrm{w}}^{\mathrm{med}}\left(\frac{Z}{A}\right)_{\mathrm{w}}^{\mathrm{med}}
$$

and the $I$-value of a given medium is obtained from the Bragg additivity rule [16]

$$
\ln I_{\text {med }}=\sum_{i} \lambda_{i} \ln I_{i}
$$

From $I_{\text {med}}$, one can determine the heavy-ion stopping power (in $\mathrm{MeV} / \mathrm{cm}$ ) using Bethe's formula [7]:

$$
S=\rho_{e} \frac{k_{0} z^{2}}{\beta^{2}}\left[\ln \left(\frac{2 m_{e} c^{2} \beta^{2}}{I_{\text {med }}\left(1-\beta^{2}\right)}\right)-\beta^{2}\right],
$$

with $k_{0}=0.17045 \mathrm{MeV} / \mathrm{cm}, z$ is the charge of the particle (e.g., $z=1$ for protons), $m_{e} c^{2}$ the rest mass energy of the electron and $\beta$ the proton velocity relative to the speed of light in vacuum.

The HU-ED and HU-SP calibration curves are finally obtained by making a fit through ICRP data points describing the relation between theoretical HU (i.e., equation 4.6 and 4.7 ) and the physical parameter or quantity in question (i.e., equations 4.8 or 4.10$)$.

\subsubsection{Electronic cross section parametrization}

For elements, the linear attenuation coefficient is given by the following formula [19]:

$$
\mu(Z)=n_{e} \sigma_{e}(Z)
$$


Here $\mu$ is the linear attenution coefficient $\left(\mathrm{in}^{-1}\right), Z$ is the atomic number and $n_{e}$ is the electronic density (in el $\left./ \mathrm{cm}^{3}\right)$. The electronic cross section $\sigma_{e}$ (in $\mathrm{cm}^{2} / \mathrm{el}$ ) is defined as the ratio of the atomic cross section $\sigma_{a}$ over the atomic number $Z$, given a photon energy or spectrum:

$$
\sigma_{e}(Z) \equiv \frac{\sigma_{a}(Z)}{Z}=\sum_{j=1}^{3} \frac{\sigma_{a, j}(Z)}{Z} .
$$

where the index $j$ represents the interaction type, i.e., Rayleigh scattering, Photoelectric effect and Compton scattering for $j=1,2,3$ respectively. Here, the electronic cross section is averaged over the energy spectrum $s(E)$ (given in $\mathrm{MeV}^{-1}$ with an implicit norm of 1) as follows

$$
\sigma_{e}(Z) \equiv \int_{0}^{E_{\max }} \sigma_{e}(E, Z) s(E) d E
$$

For a monoenergetic spectrum, one can use $s(E)=\delta\left(E-E_{0}\right)$, the Dirac delta function centered at $E_{0}$, in equation 4.13 without loss of generality. Let us define the parametric electronic cross section $\hat{\sigma}_{e}$ for any $Z$ (integers or non-integers) as follows

$$
\hat{\sigma}_{e}(Z)=\sum_{m=1}^{M} a_{m} Z^{m-1} .
$$

with $Z \in \mathbb{R}$ and $Z \geq 1$. Here the coefficients $a_{m}$ are obtained using a least square fit on XCOM cross section data [6] for a given spectrum over a range $Z \in\left[Z_{\min }, Z_{\max }\right]$ and $M$ is a user-defined parameter establishing the level of accuracy of the fit. Figure 4-1 shows the behaviour of electron cross section as a function of $Z$ for all four spectra of the Siemens SOMATOM Definition Flash DSCT: 80 kVp, 100 kVp, 140 kVp and 


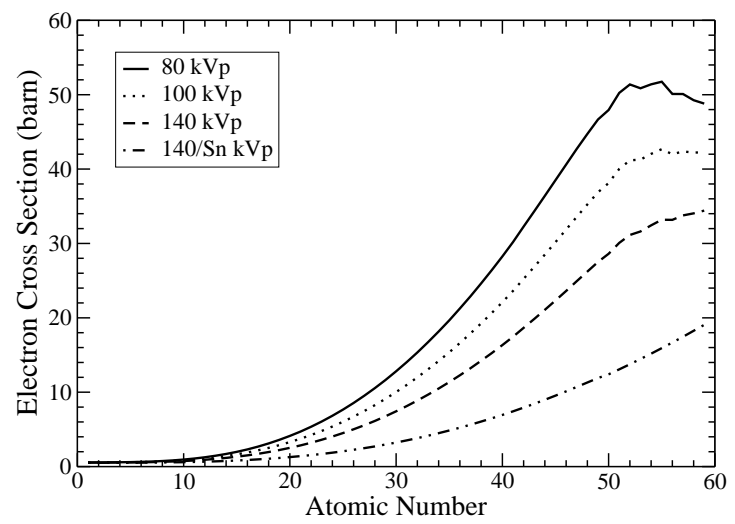

Figure 4-1: XCOM electron cross section averaged over the spectra of the Siemens SOMATOM Definition Flash DSCT as a function of the atomic number.

140/Sn kVp (i.e., $140 \mathrm{kVp}$ with $\mathrm{Sn}$ filter). The curves are bijective for all spectra for $1 \leq Z \leq 52$

\subsubsection{Mixtures}

For a given energy or energy spectrum, the mass attenuation coefficient of a mixture is given by the following relation[19]

$$
\left(\frac{\mu}{\rho}\right)_{\text {med }}=\sum_{i} w_{i}\left(\frac{\mu}{\rho}\right)_{i},
$$

with $w_{i}$ the relative fractional weight. By analogy, one can show that the electronic cross section of a mixture is given by

$$
\sigma_{e, \text { med }}=\sum_{i} \lambda_{i} \sigma_{e}\left(Z_{i}\right)
$$

for a given energy or spectrum, with $\lambda_{i}$ the relative fraction of electrons of the $i^{t h}$ element in the mixture as defined in equation 4.4 . 


\subsubsection{Effective atomic number}

As described by equation 4.11, the probability for photons to interact with matter is characterized by the electronic cross section. While for elements, the electronic cross section for a given energy or spectrum can be entirely characterized by the atomic number, a judicious definition of effective atomic number for mixtures can be useful for quantifying photon interactions with respect to elements. This yields the following definition.

The effective atomic number of a mixture, noted $Z_{\text {med }}$, is defined such that for a given energy spectrum, the parametric electronic cross section evaluated at $Z_{\text {med }}$ equals the electronic cross section of the mixture averaged over the spectrum $\sigma_{e, \text { med }}$. Mathematically, this means that there exists a $Z_{\text {med }}$ such that

$$
\hat{\sigma}_{e}\left(Z_{\text {med }}\right)=\sigma_{e, \text { med }}
$$

Since this definition can only yield a unique $Z$ if equation 4.14 is bijective, one must limit the domain of $Z_{\text {med }}$ when performing the fit for a given $s(E)$ to avoid either discontinuities or non monotonicity due to the evolution of the atomic structure with $Z$. Defining the domain $Z_{\min } \leq Z \leq Z_{\max }$ where $\hat{\sigma}_{e}(Z)$ is bijective, the effective atomic number is defined by

$$
Z_{\text {med }} \equiv \hat{\sigma}_{e}^{-1}\left(\sigma_{e, \text { med }}\right)
$$

Note here that with this general definition, $Z_{\text {med }}$ depends on the elemental composition as well as the photon energy spectrum $s(E)$ (except for elements). As shown 
in figure $4-1$, the definition of $Z_{\text {med }}$ is valid for $1 \leq Z \leq 52$ over the spectra used in the present study.

Figure 4-2 shows examples of calculated $Z_{\text {med }}$ for 4 different ICRP tissue compositions using equation 4.18 as a function of energy including 7 spectra from 2 different scanners (i.e., scanner 1: Siemens SOMATOM Flash Definition, scanner 2: Philips Gemini GXL). In the range of CT imaging energies, $Z_{\text {med }}$ varies weakly with the energy spectrum (or $\mathrm{kVp}$ ). It is therefore convenient to define a fixed value of effective atomic number and consider the variation of $Z_{\text {med }}$ over relevant energies as a non statistical uncertainty component due to the theoretical flaw of defining a fixed effective atomic number.

For each tissue composition, the fixed value of $Z_{\text {med }}$ is defined as

$$
\bar{Z}_{\text {med }}=\frac{\max \left\{Z_{\text {med }}\right\}+\min \left\{Z_{\text {med }}\right\}}{2}
$$

where the minimum and maximum values of calculated $Z_{\text {med }}$ are taken over the range of CT imaging spectra. The variation of the effective atomic number is defined as

$$
\Delta Z_{\text {med }}=\frac{\max \left\{Z_{\text {med }}\right\}-\min \left\{Z_{\text {med }}\right\}}{2}
$$

Tables $4-1$ and $4-2$ provide fixed $Z_{\text {med }}$ values with their variation (i.e., $\bar{Z}_{\text {med }} \pm$ $\Delta Z_{\text {med }}$ ) for the Gammex 467 phantom and ICRP tissues respectively using the spectra of the Siemens SOMATOM Definition Flash DSCT. The values computed with the spectra of the Philips Gemini GXL are typically included in the range of the Siemens SOMATOM Definition Flash data, with the exception of bones where the differences in fixed $Z_{\text {med }}$ for both scanners are less than 0.008 . 

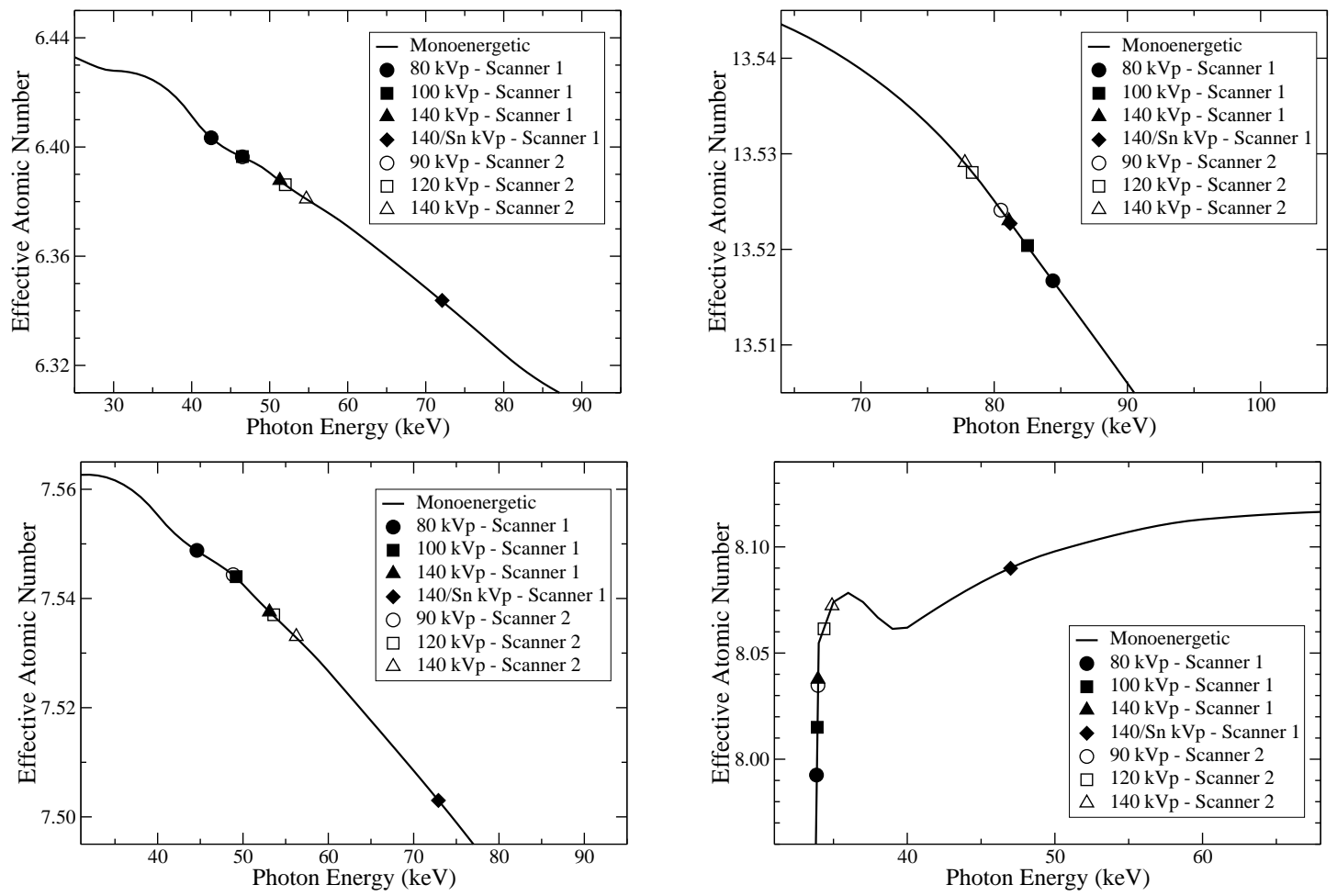

Figure 4-2: Effective atomic number values calculated as a function energy from the XCOM database for ICRP tissues: adipose tissue (top left), cortical bone (top right), muscle (bottom left) and thyroid (bottom right). Note that scanner 1 refers to the Siemens SOMATOM Definition Flash and scanner 2 refers to the Philips Gemini GXL. 


\begin{tabular}{lcccc}
\hline Material & $\rho_{e}$ & $Z_{\text {med }}$ & $I(\mathrm{eV})$ & $\hat{I}(\mathrm{eV})$ \\
\hline Water & 1.00 & $7.45 \pm 0.02$ & 75.3 & 74.2 \\
LN300 Lung RMI-455 & 0.29 & $7.55 \pm 0.03$ & 73.9 & 74.7 \\
LN450 Lung RMI-485 & 0.48 & $7.52 \pm 0.03$ & 73.8 & 74.5 \\
AP6 Adipose tissue RMI-453 & 0.93 & $6.17 \pm 0.02$ & 66.6 & 61.9 \\
BR12 Breast RMI-454 & 0.96 & $6.87 \pm 0.03$ & 68.2 & 70.2 \\
CT Solid Water RMI-451 & 0.99 & $7.66 \pm 0.03$ & 70.4 & 75.2 \\
LV1 RMI-482 & 1.06 & $7.66 \pm 0.03$ & 70.3 & 75.2 \\
SR2 Brain RMI-481 & 1.05 & $6.04 \pm 0.03$ & 63.5 & 59.9 \\
CB2 - 30\% CaCO 3 RMI-484 & 1.28 & $10.76 \pm 0.02$ & 80.7 & 83.1 \\
CB2 - 50\% CaCO 3 RMI-480 & 1.47 & $12.40 \pm 0.01$ & 93.2 & 98.0 \\
SB3 Bone, Cortical RMI-450 & 1.70 & $13.51 \pm 0.01$ & 104.5 & 111.7 \\
B200 Bone Mineral RMI-487 & 1.10 & $10.29 \pm 0.03$ & 80.2 & 80.9 \\
IB3 Inner Bone RMI-456 & 1.09 & $10.28 \pm 0.03$ & 80.1 & 80.9 \\
\hline
\end{tabular}

Table 4-1: Summary of the material parameters of the Gammex 467 phantom computed from mass densities and elemental compositions provided by the manufacturer (for reference use only). 


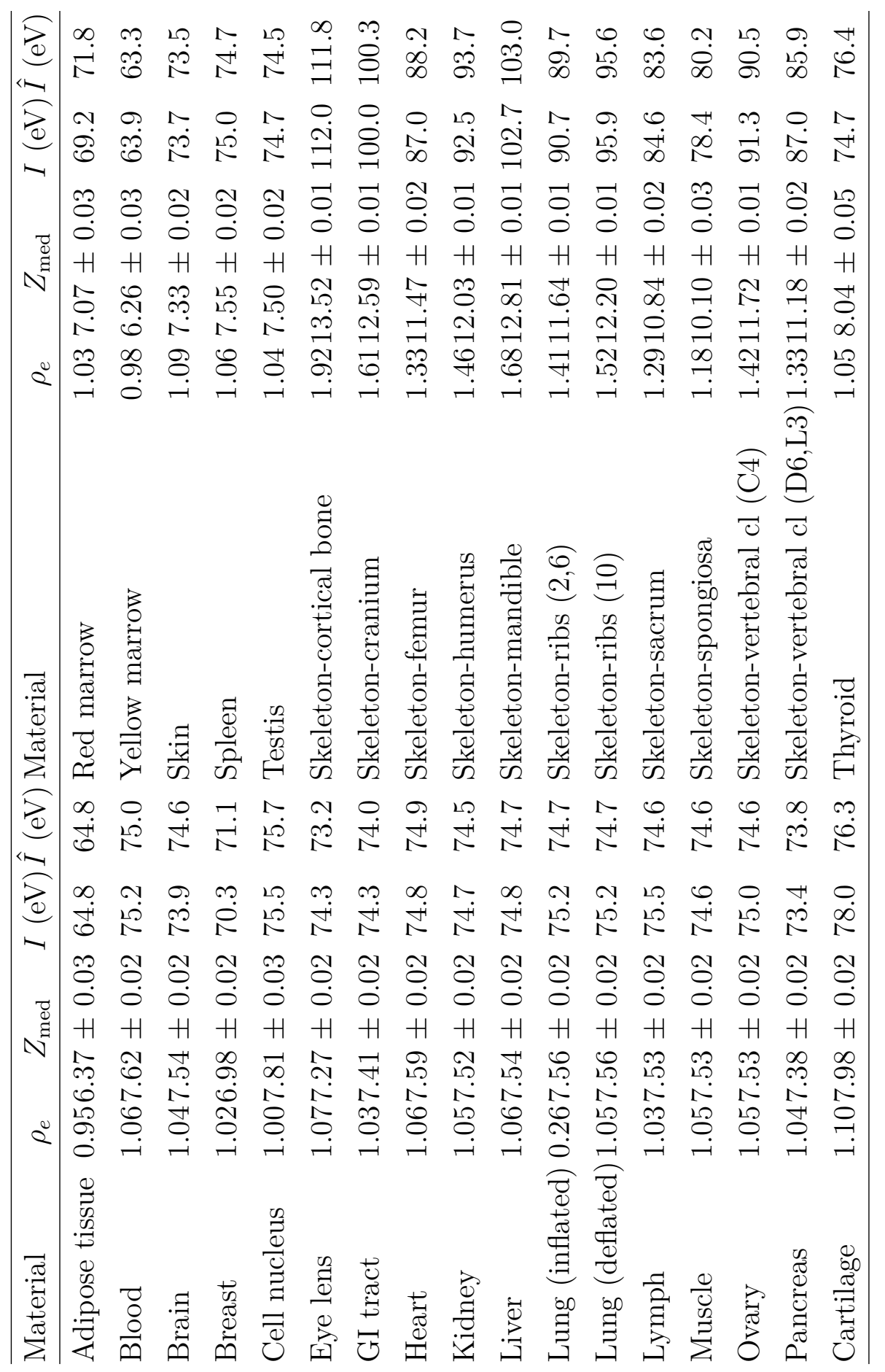

Table 4-2: Summary of human tissue parameters computed from mass densities and elemental compositions recommended by the ICRP [42]. 


\subsubsection{Mean excitation energy parametrization}

Due to the behaviour of the atomic structure with $Z$, a robust relationship between the mean excitation energy and the effective atomic number cannot be established for all materials. As proposed by Yang et al. (2010), the $I$-value can be parametrized as a function of the effective atomic number for a restrained set of media with sufficient accuracy with respect to tabulated data uncertainties. Based on the present definition of $Z_{\text {med }}$, one can determine the following relationship for ICRP tissues:

$$
\hat{I}(Z)=\left\{\begin{array}{ll}
e_{1} Z+e_{2} & \text { for } Z<Z_{\min } \\
e_{3} Z^{5}+e_{4} Z^{4}+e_{5} Z^{3}+e_{6} Z^{2}+e_{7} Z+e_{8} & \text { for } Z_{\min } \leq Z \leq Z_{\max } \\
e_{9} Z+e_{10} & \text { for } Z>Z_{\max }
\end{array} .\right.
$$

where $e_{1}=14.007762, e_{2}=-24.414214, e_{3}=-0.005342, e_{4}=0.207079, e_{5}=$ $-2.589844, e_{6}=8.339473, e_{7}=51.895887, e_{8}=-219.722173, e_{9}=11.794847$, $e_{10}=-47.707141, Z_{\min }=6.26$ and $Z_{\max }=13.52$. The central portion of the function described by equation 4.21 is obtained using a least square fit on the dataset. The two other sections of the function are extrapolated linearly to make the relation continuous over $Z$ and assuming that the slope of the function remains constant beyond $Z_{\min }$ and $Z_{\max }$. In the central region of the fit, a proper variance analysis of the fit yields a mean uncertainty below $\pm 1 \mathrm{eV}(1 \sigma$ level), which is negligible as compared to reported uncertainties on ICRU data [16].

Tables 4-1 and 4-2 provide $I$-values determined with the Bragg additivity rule [16] and equation 4.21 for the Gammex 467 phantom and ICRP tissues respectively. 


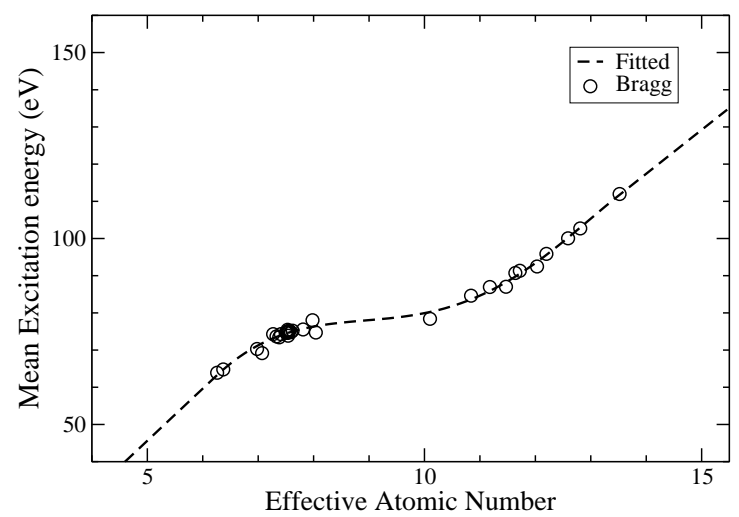

Figure 4-3: Parametrization of the ICRU mean excitation energy as a function of the EAN defined in this paper for ICRP tissues.

The elemental $I$-values used in the additivity rule are the ones recommended by the ICRU report 37 [16] and are shown in table 4-3. The differences between fitted and ICRU-recommended $I$-values shown in table $4-1$ for the Gammex 467 phantom are much higher that the uncertainty on the fit (i.e., as high as $7 \mathrm{eV}$ ) due to the fact that these data are not included in equation 4.21 .

\begin{tabular}{|c|c|c|c|c|c|}
\hline Element symbol & Atomic number & $I(e V)$ & Element symbol & Atomic number & $I(e V)$ \\
\hline $\mathrm{H}$ & 1 & 19.2 & $\mathrm{P}$ & 15 & 195.5 \\
\hline $\mathrm{C}$ & 6 & 81.0 & $\mathrm{~S}$ & 16 & 203.4 \\
\hline $\mathrm{N}$ & 7 & 82.0 & $\mathrm{Cl}$ & 17 & 180.0 \\
\hline $\mathrm{O}$ & 8 & 106.0 & $\mathrm{~K}$ & 19 & 214.7 \\
\hline $\mathrm{Na}$ & 11 & 168.4 & $\mathrm{Ca}$ & 20 & 215.8 \\
\hline $\mathrm{Mg}$ & 12 & 176.3 & $\mathrm{Fe}$ & 26 & 323.2 \\
\hline $\mathrm{Si}$ & 14 & 195.5 & $\mathrm{I}$ & 53 & 554.8 \\
\hline
\end{tabular}

Table 4-3: Recommended values of mean excitation energies to determine the $I$ values of mixtures using Bragg's additivity rule [16]. 


\subsubsection{Extension of the SECT stoichiometric calibration}

In order to develop a rigorous formalism for DECT based on a stoichiometric calibration, the method of Schneider et al. (1996) needs to be extended using a more robust cross section parametrization. Combining the parameterization of equation 4.14 with equation 4.11, the relationship of Schneider et al. (1996) described at equation 4.6 can be extended to the following for a given $\mathrm{kVp}$

$$
u=\rho_{e} f\left(Z_{\text {med }}\right)
$$

where $u$ is defined in equation $4.7, Z_{\text {med }}$ is the effective atomic number defined in equation 4.18 and $f$ is a function given by

$$
f(Z) \equiv \sum_{m=1}^{M} b_{m} Z^{m-1}
$$

Here $M$ is a user-defined parameter establishing the level of accuracy of the relation. Note that, by definition, equation 4.23 is linked to equation 4.14 by $b_{m} \equiv \frac{a_{m}}{\sigma_{e, \mathrm{w}}}$.

In practice, the photon spectrum throughout the scanned media is not known accurately and, therefore, the coefficients $b_{m}$ are obtained using a least square fit of the following system based on the set of measurements $\mathrm{HU}=\left\{\mathrm{HU}_{n}\right\}$, the array containing the measured $\mathrm{HU}$ of the $N$ distinctive media:

$$
\mathbf{u}=\mathbf{F b}
$$


with $\mathbf{u}$ the $N$-elements array with $u_{n}=\frac{\mathrm{HU}_{n}+1000}{1000}$ and $\mathbf{F}$ a matrix of dimensions $N \times M$ with elements $F_{n, m}=\rho_{e, n} Z_{\text {med }, n}^{m-1}$, that is

$$
\mathbf{F} \equiv\left(\begin{array}{cccc}
\rho_{e, 1} & \rho_{e, 1} Z_{\mathrm{med}, 1} & \ldots & \rho_{e, 1} Z_{\mathrm{med}, 1}^{M-1} \\
\rho_{e, 2} & \rho_{e, 2} Z_{\mathrm{med}, 2} & \ldots & \rho_{e, 2} Z_{\mathrm{med}, 2}^{M-1} \\
\vdots & \vdots & \ddots & \vdots \\
\rho_{e, N} & \rho_{e, N} Z_{\mathrm{med}, N} & \ldots & \rho_{e, N} Z_{\mathrm{med}, N}^{M-1}
\end{array}\right)
$$

The least square solution of the coefficients $b_{m}$ is given by

$$
\mathbf{b} \approx \hat{\mathbf{b}}=\left(\mathbf{F}_{\text {cal }}^{\mathrm{T}} \mathbf{F}_{\text {cal }}\right)^{-\mathbf{1}} \mathbf{F}_{\text {cal }}^{\mathrm{T}} \mathbf{u}_{\text {cal }}
$$

with $\mathbf{F}_{\text {cal }}$ obtained with medium data of the calibration phantom and $\mathbf{u}_{\text {cal }}$ the corresponding measurements. Finally, the theoretical values of HU for human tissues are estimated with

$$
\hat{u}_{\mathrm{ICRP}}=\rho_{e, \mathrm{ICRP}} \sum_{m=1}^{M} \hat{b}_{m} Z_{\mathrm{ICRP}}^{m-1} .
$$

\subsubsection{DECT stoichiometric calibration}

Let there be two sets of $\mathrm{CT}$ data $(\mathrm{L}=$ low $\mathrm{kVp}$ and $\mathrm{H}=$ high $\mathrm{kVp})$, noted $\mathrm{HU}_{\mathrm{L}}=\left\{\mathrm{HU}_{\mathrm{L}, 1}, \ldots, \mathrm{HU}_{\mathrm{L}, N}\right\}$ and $\mathrm{HU}_{\mathrm{H}}=\left\{\mathrm{HU}_{\mathrm{H}, 1}, \ldots, \mathrm{HU}_{\mathrm{H}, N}\right\}$, taken simultaneously with two distinctive energies (or $\mathrm{kVp}$ ). Using equation 4.7, for each voxel one can write

$$
\begin{aligned}
& u_{\mathrm{L}}=\frac{\mathrm{HU}_{\mathrm{L}}+1000}{1000} \\
& u_{\mathrm{H}}=\frac{\mathrm{HU}_{\mathrm{H}}+1000}{1000}
\end{aligned}
$$


To define an index independent of the relative electron density and depending on the effective atomic number, the two following definitions are appropriate. The index $\Gamma$ encompass both definitions and is written as follows

$$
\Gamma=\left\{\begin{array}{cc}
\frac{u_{\mathrm{L}}-u_{\mathrm{H}}}{u_{\mathrm{L}}+u_{\mathrm{H}}} & \text { for } \Gamma=\mathrm{DEI} \\
\frac{u_{\mathrm{L}}}{u_{\mathrm{H}}} & \text { for } \Gamma=\mathrm{DER}
\end{array} .\right.
$$

Here DEI stands for dual energy index (e.g., ref. [21]) while DER stands for dual energy ratio. Since $\Gamma$ is independent of the electron density and assuming that $Z_{\text {med }}$ is independent of the photon energy spectrum over the range used, one can write $\Gamma \equiv \Gamma(Z)$ over a domain $Z \in\left[Z_{\min }, Z_{\max }\right]$ where $\Gamma$ and $Z$ are bijective. Therefore one can write

$$
Z=\sum_{k=1}^{K} c_{k} \Gamma^{k-1}
$$

with $K$ a user-defined parameter establishing the level of accuracy of the relationship. The equation can be written using the following matrix system:

$$
\mathrm{Z}=\Gamma \mathbf{c}
$$


with $\mathbf{Z}$ the $N$-elements array with $Z_{n}=Z_{\mathrm{med}, n}$ and $\boldsymbol{\Gamma}$ a matrix of dimensions $N \times K$ with elements $\Gamma_{n, k}=\Gamma_{n}^{k-1}$, that is

$$
\boldsymbol{\Gamma} \equiv\left(\begin{array}{cccc}
1 & \Gamma_{1} & \cdots & \Gamma_{1}^{K-1} \\
1 & \Gamma_{2} & \cdots & \Gamma_{2}^{K-1} \\
\vdots & \vdots & \ddots & \vdots \\
1 & \Gamma_{N} & \cdots & \Gamma_{N}^{K-1}
\end{array}\right) .
$$

The least square solution of the coefficients $c_{k}$ is then given by

$$
\mathbf{c} \approx \hat{\mathbf{c}}=\left(\boldsymbol{\Gamma}_{\text {cal }}^{\mathrm{T}} \boldsymbol{\Gamma}_{\text {cal }}\right)^{-\mathbf{1}} \boldsymbol{\Gamma}_{\text {cal }}{ }^{\mathrm{T}} \mathbf{Z}_{\text {cal }},
$$

with $\boldsymbol{\Gamma}_{\text {cal }}$ and $\mathbf{Z}_{\text {cal }}$ obtained respectively from measurements and medium data using the calibration phantom.

For a post-calibration measurement, the estimator of the effective atomic number is given by

$$
\hat{Z}=\sum_{k=1}^{K} \hat{c}_{k} \Gamma_{\text {meas }}^{k-1},
$$

with $\Gamma_{\text {meas }}$ the post-calibration measured index (i.e., DEI or DER) and $K$ a userdefined parameter establishing the level of accuracy of the relationship.

Using the relationship established at equations 4.22-4.23, the estimator of the electron density is defined using the following maximum likelihood estimation

$$
\hat{\rho}_{e}=\frac{1}{2}\left[\hat{\rho}_{e, \mathrm{~L}}+\hat{\rho}_{e, \mathrm{H}}\right],
$$


with

$$
\hat{\rho}_{e, \mathrm{~L} / \mathrm{H}} \equiv \frac{u_{\mathrm{L} / \mathrm{H}}}{\sum_{m=1}^{M} \hat{b}_{\mathrm{L} / \mathrm{H}, m} \hat{Z}^{m-1}},
$$

where the subscript L/H represents L or H. Here the coefficients $\hat{b}_{\mathrm{L}, m}$ and $\hat{b}_{\mathrm{H}, m}$ are estimated for the two distinctive sets of CT data.

\subsubsection{Variance analysis}

This section aims to derive analytic expressions for evaluating uncertainties of the estimated physical parameters during the stoichiometric calibration. There are two situations of interest for evaluating uncertainties: 1) the stoichiometric calibration, and 2) clinical situations. During the stoichiometric calibration, uncertainties are smaller since $\mathrm{HU}$ values are averaged over a region of interest constituted uniformly (typically a few $\mathrm{cm}^{3}$ ). In clinical situations, HU values are reported to single voxels (i.e., less than $1 \mathrm{~mm}^{3}$ ) and their uncertainty is dominated by noise, typically about 4-10 times the uncertainty on the average $\mathrm{HU}$ value. As it is shown in this section, the uncertainty on all physical parameters studied is directly proportional to the uncertainty on HU and therefore the uncertainty on given parameters in clinical situations are about 4-10 times higher than for the calibration.

\subsubsection{Statistical uncertainties on experimental measurements}

The uncertainty on the estimation of the electron density and the effective atomic number depends entirely on the statistical behaviour of the sets $u_{\mathrm{L}}$ and $u_{\mathrm{H}}$ during the measurement (i.e., $u_{\mathrm{L}, \text { meas }}$ and $u_{\mathrm{H} \text {,meas }}$ ). One simple way to evaluate the uncertainty on these datasets is to assume that the variables behave normally with an uncertainty 
being constant over the HU domain. This yields to the following relationship[35]

$$
\sigma_{\mathrm{L} / \mathrm{H}, \text { meas }} \approx \sqrt{\frac{1}{\nu} \sum_{i=1}^{N}\left(u_{\mathrm{L} / \mathrm{H}, \text { meas }, i}-\hat{u}_{\mathrm{L} / \mathrm{H}, i}\right)^{2}},
$$

where the subscript $\mathrm{L} / \mathrm{H}$ represents $\mathrm{L}$ or $\mathrm{H}$ and $\nu$ is the number of degrees of freedom of the fit (i.e., $\nu=N-M)$. The estimated values of reduced HU for the calibration phantom are evaluated applying equation 4.27 to the proper materials. However, for clinical measurements one can simply assume the uncertainty to be dominated by noise, that is

$$
\sigma_{\mathrm{L} / \mathrm{H}, \mathrm{clin}} \approx \sigma_{\mathrm{L} / \mathrm{H}, \text { noise }}
$$

To derive an analytic expression of the uncertainty of $\hat{Z}$ and $\hat{\rho}_{e}$, one can make the following approximation. While the uncertainty on the estimation depends on fits (i.e., $\hat{u}_{\mathrm{L}}$ and $\hat{u}_{\mathrm{H}}$ ) and measurements (i.e., $u_{L \text {,meas }}$ and $u_{H \text {,meas }}$ ), the uncertainty contribution from the fit is small as compared to the measurement uncertainty when a large number of material is used in the stoichiometric calibration (e.g. $N>12$, see ref. [8]). Therefore, one can assume that the coefficients $\hat{b}_{\mathrm{L}, m}, \hat{b}_{\mathrm{H}, m}, \hat{c}_{\mathrm{L}, m}$ and $\hat{c}_{\mathrm{H}, m}$ have a negligible contribution to the uncertainty and this simplifies the variance analysis considerably.

\subsubsection{Statistical uncertainties on estimated parameters}

Applying the rule of error propagation to equations 4.34 and 4.35 , the uncertainties are given by the following relations (see Appendix A):

$$
\sigma_{\hat{Z}} \approx \begin{cases}\frac{2\left|\sum_{k=1}^{K}(k-1) \hat{c}_{k} \Gamma^{k-2}\right| \sqrt{u_{\mathrm{H}}^{2} \sigma_{\mathrm{L}}^{2}+u_{\mathrm{L}}^{2} \sigma_{\mathrm{H}}^{2}}}{\left(u_{\mathrm{L}}+u_{\mathrm{H}}\right)^{2}} & \text { for } \Gamma=\mathrm{DEI} \\ \frac{\left|\sum_{k=1}^{K}(k-1) \hat{c}_{k} \Gamma^{k-2}\right| \sqrt{u_{\mathrm{H}}^{2} \sigma_{\mathrm{L}}^{2}+u_{\mathrm{L}}^{2} \sigma_{\mathrm{H}}^{2}}}{u_{\mathrm{H}}^{2}} & \text { for } \Gamma=\mathrm{DER}\end{cases}
$$


and

$$
\sigma_{\hat{\rho}_{e}} \approx\left\{\begin{array}{ll}
\frac{1}{2} \sqrt{\left[\frac{\hat{\rho}_{e, \mathrm{~L}}}{u_{\mathrm{L}}}-\frac{2 A u_{\mathrm{H}}}{\left(u_{\mathrm{L}}+u_{\mathrm{H}}\right)^{2}}\right]^{2} \sigma_{\mathrm{L}}^{2}+\left[\frac{\hat{\rho}_{e, \mathrm{H}}}{u_{\mathrm{H}}}+\frac{2 A u_{\mathrm{L}}}{\left(u_{\mathrm{L}}+u_{\mathrm{H}}\right)^{2}}\right]^{2} \sigma_{\mathrm{H}}^{2}} & \text { for } \Gamma=\mathrm{DEI} \\
\frac{1}{2} \sqrt{\left[\frac{\hat{\rho}_{e, \mathrm{~L}}}{u_{\mathrm{L}}}-\frac{A}{u_{\mathrm{H}}}\right]^{2} \sigma_{\mathrm{L}}^{2}+\left[\frac{\hat{\rho}_{e, \mathrm{H}}}{u_{\mathrm{H}}}+\frac{A u_{\mathrm{L}}}{u_{\mathrm{H}}^{2}}\right]^{2} \sigma_{\mathrm{H}}^{2}} & \text { for } \Gamma=\mathrm{DER}
\end{array},\right.
$$

where

$$
A \equiv\left[\sum_{m=1}^{M}(m-1)\left(\frac{\hat{\rho}_{e, \mathrm{~L}}^{2}}{u_{\mathrm{L}}} \hat{b}_{\mathrm{L}, m}+\frac{\hat{\rho}_{e, \mathrm{H}}^{2}}{u_{\mathrm{H}}} \hat{b}_{\mathrm{H}, m}\right) \hat{Z}^{m-2}\right]\left[\sum_{k=1}^{K}(k-1) \hat{c}_{k} \Gamma^{k-2}\right] .
$$

Note here that $u_{\mathrm{L}}$ and $u_{\mathrm{H}}$ are assumed statistically independent. However, $\hat{\rho}_{e}$ and $\hat{Z}$ are correlated and their covariance is given as follows (see Appendix A). For $\Gamma=$ DEI,

$$
\begin{aligned}
\sigma_{\hat{\rho}_{e}, \hat{Z}} \approx & {\left[\sum_{k=1}^{K}(k-1) \hat{c}_{k} \Gamma^{k-2}\right]\left[\frac{u_{\mathrm{H}} \sigma_{\mathrm{L}}^{2}}{\left(u_{\mathrm{L}}+u_{\mathrm{H}}\right)^{2}}\left[\frac{\hat{\rho}_{e, \mathrm{~L}}}{u_{\mathrm{L}}}-\frac{2 A u_{\mathrm{H}}}{\left(u_{\mathrm{L}}+u_{\mathrm{H}}\right)^{2}}\right]\right] } \\
& -\left[\sum_{k=1}^{K}(k-1) \hat{c}_{k} \Gamma^{k-2}\right]\left[\frac{u_{\mathrm{L}} \sigma_{\mathrm{H}}^{2}}{\left(u_{\mathrm{L}}+u_{\mathrm{H}}\right)^{2}}\left[\frac{\hat{\rho}_{e, \mathrm{H}}}{u_{\mathrm{H}}}+\frac{2 A u_{\mathrm{L}}}{\left(u_{\mathrm{L}}+u_{\mathrm{H}}\right)^{2}}\right]\right],
\end{aligned}
$$

and for $\Gamma=\mathrm{DER}$,

$$
\sigma_{\hat{\rho}_{e}, \hat{Z}} \approx\left[\sum_{k=1}^{K}(k-1) \hat{c}_{k} \Gamma^{k-2}\right]\left[\frac{\sigma_{\mathrm{L}}^{2}}{2 u_{\mathrm{H}}}\left[\frac{\hat{\rho}_{e, \mathrm{~L}}}{u_{\mathrm{L}}}-\frac{A}{u_{\mathrm{H}}}\right]-\frac{u_{\mathrm{L}} \sigma_{\mathrm{H}}^{2}}{2 u_{\mathrm{H}}^{2}}\left[\frac{\hat{\rho}_{e, \mathrm{H}}}{u_{\mathrm{H}}}+\frac{A u_{\mathrm{L}}}{u_{\mathrm{H}}^{2}}\right]\right] .
$$

The uncertainty on the estimated $I$-value is given by the following rule of error propagation

$$
\sigma_{\hat{I}} \approx\left|\frac{\partial \hat{I}}{\partial Z}\right| \sigma_{\hat{Z}}
$$


with the derivative of $\hat{I}$ estimated from equation 4.21 as follows:

$$
\frac{\partial \hat{I}}{\partial Z}=\left\{\begin{array}{cl}
e_{1} & \text { for } Z<Z_{\min } \\
5 e_{3} Z^{4}+4 e_{4} Z^{3}+3 e_{5} Z^{2}+2 e_{6} Z+e_{7} & \text { for } Z_{\min } \leq Z \leq Z_{\max } \\
e_{9} & \text { for } Z>Z_{\max }
\end{array}\right.
$$

The uncertainty on heavy-ion stopping power is given by the following relation (see Appendix B):

$$
\sigma_{\hat{S}} \approx \sqrt{\left[\frac{\hat{S}}{\rho_{e}}\right]^{2} \sigma_{\hat{\rho}_{e}}^{2}+\left[-\frac{\rho_{e}}{\hat{I}} \frac{k_{0} z^{2}}{\beta^{2}} \frac{\partial \hat{I}}{\partial Z}\right]^{2} \sigma_{\hat{Z}}^{2}-2\left[\frac{\hat{S}}{\rho_{e}}\right]\left[\frac{\rho_{e}}{\hat{I}} \frac{k_{0} z^{2}}{\beta^{2}} \frac{\partial \hat{I}}{\partial Z}\right] \sigma_{\hat{\rho}_{e}, \hat{Z}}}
$$

Finally, the uncertainty on the range of heavy-ions is approximately equal to (see Appendix C):

$$
\sigma_{\hat{R}} \approx \sqrt{\hat{R} \Delta x} \sqrt{\frac{\overline{\sigma_{S}^{2}}}{\bar{S}^{2}}}
$$

where $R$ is the range (in $\mathrm{mm}$ ), $\Delta x$ is average size of a CT-reconstructed voxel in the

direction of the beam (in $\mathrm{mm}$ ), $\overline{\sigma_{S}^{2}}$ is the average variance of the stopping power and $\bar{S}$ is the average stopping power, both quantities averaged over the ion trajectory.

\subsubsection{Non statistical uncertainties}

Since $Z_{\text {med }}$ is by definition energy-dependent, an additional uncertainty component, which cannot be determined using statistical analysis, must be considered when applying the DECT formalism. In tables $4-1$ and $4-2$, values of $Z_{\text {med }}$ and $\Delta Z_{\text {med }}$ are given for different phantom materials. These values are calculated using equations 4.19 and 4.20 over the spectra of the Siemens SOMATOM Definition Flash and the variation $\Delta Z_{\text {med }}$ can be treated as a non statistical uncertainty due to the theoretical 
flaw of defining a fixed effective atomic number. Therefore, the overall uncertainty (statistical an non statistical) of $\hat{Z}$ and $\hat{\rho}_{e}$ can be written as follows:

$$
\sigma_{\hat{Z}, \text { overall }} \approx \sqrt{\sigma_{\hat{Z}}^{2}+\Delta \hat{Z}^{2}}
$$

and

$$
\sigma_{\hat{\rho}_{e}, \text { overall }} \approx \sqrt{\sigma_{\hat{\rho}_{e}}^{2}+\Delta \hat{\rho}_{e}^{2}}
$$

Here $\Delta \hat{Z}$ is taken from tables $4-1$ and $4-2$ as $\Delta \hat{Z} \approx \Delta Z_{\text {med }}$.

Finally, $\Delta \hat{\rho}_{e}$ is evaluated as follows

$$
\Delta \hat{\rho}_{e}=\frac{1}{2}\left|\frac{u_{\mathrm{L}}\left[\sum_{m=1}^{M}(m-1) \hat{b}_{\mathrm{L}, m} \hat{Z}^{m-2}\right]}{\left[\sum_{m=1}^{M} \hat{b}_{\mathrm{L}, m} \hat{Z}^{m-1}\right]^{2}}+\frac{u_{\mathrm{H}}\left[\sum_{m=1}^{M}(m-1) \hat{b}_{\mathrm{H}, m} \hat{Z}^{m-2}\right]}{\left[\sum_{m=1}^{M} \hat{b}_{\mathrm{H}, m} \hat{Z}^{m-1}\right]^{2}}\right| \Delta \hat{Z}
$$

assuming $\frac{\Delta_{\hat{\rho}_{e}}}{\Delta \hat{Z}} \approx\left|\frac{\partial \hat{\rho}_{e}}{\partial \hat{Z}}\right|$.

\subsection{Materials and methods}

\subsubsection{Theoretical framework}

In order to evaluate the robustness of the formalism without the presence of artefacts such as scatter, noise and beam hardening, the stoichiometric calibration method is simulated using theoretical values of HU. The theoretical framework is based on the XCOM database and uses spectra of 80, 100, 140 and 140/Sn kVp to calculate the attenuation coefficients of the phantom materials listed in table 4-4 being used during the calibration process. While the XCOM database provides mass attenuation coefficients for elements for $Z \leq 100$ at energies from $1 \mathrm{keV}$ to $100 \mathrm{GeV}$ [6], the attenuation coefficients for mixtures and spectra are computed with equations 4.11, 4.13, 4.15 and 4.16. The linear attenuation coefficients of the phantom materials 


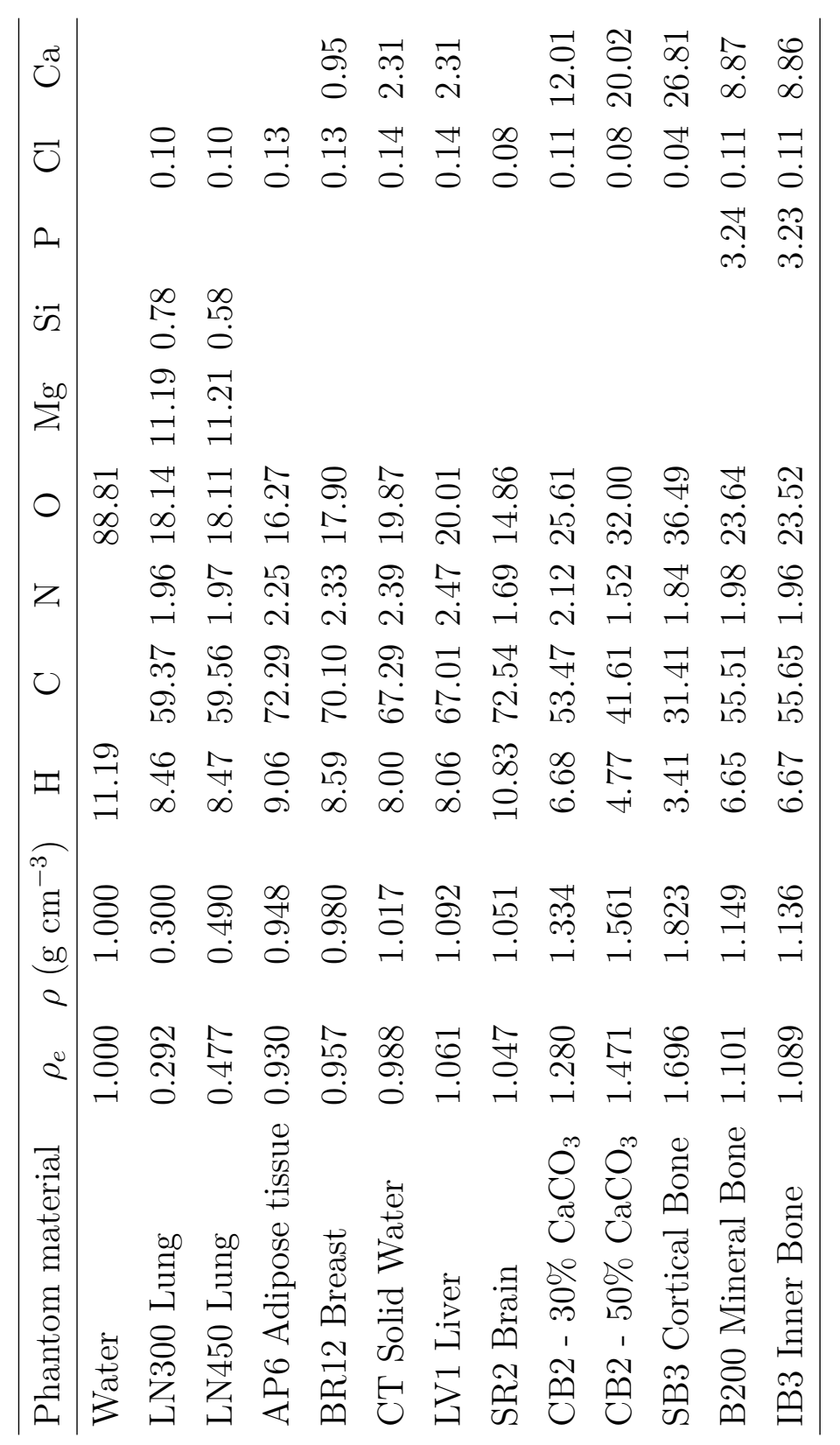

Table 4-4: Electron densities and elemental compositions of the Gammex 467 phantom materials (for reference use only). 
are then used to estimate the parameters of the model as required by equations 4.26 and 4.33 (i.e., $\left\{\hat{b}_{m}\right\}$ and $\left\{\hat{c}_{m}\right\}$ ). Finally, the stoichiometric calibration is simulated for SECT and DECT applying the model to ICRP tissue compositions (i.e., see ref. [42]), this using equation 4.27 for SECT and equations 4.34 and 4.35 for DECT.

\subsubsection{Experimental measurements}

The calibration phantom used is the Gammex 467 tissue characterization phantom, a $33 \mathrm{~cm}$ diameter Solid Water disk containing interchangeable rods of 12 human tissue substitutes and 1 vial for water measurements. The elemental compositions of the rods are summarized in table $4-4$. The relative electron densities range from 0.292 to 1.692 while the effective atomic numbers range from 6.04 to 13.51 . The phantom is scanned with a Siemens SOMATOM Definition Flash DSCT at three energy couples shown on table $4-5$ with a pitch of 0.6 and the D30f kernel in order to obtain a fast and smooth dual energy reconstruction. The parameters used during the scans are summarized in table 4-5. For each rod, the analyzed volume of interest (VOI) is constituted of 10496 voxels with dimensions of approximately $0.77 \times 0.77 \times 0.66 \mathrm{~mm}^{3}\left(\right.$ total volume $\left.\approx 3.63 \mathrm{~cm}^{3}\right)$. For the stoichiometric calibration, the reported HU values are taken as the average HU over each VOI. For the noise analysis, the HU uncertainty is taken as the standard deviation of HU over each VOI.

\subsection{Results and discussion}

\subsubsection{Theoretical self-consistency}

\section{Parametrization domain of $Z$}

With respect to the formalism, there must exists a range of $Z$ where the electron cross section and $\Gamma$ (DEI or DER) are bijective with $Z$. Using XCOM data over all 


\begin{tabular}{lccc}
\hline Energy couple & 1 & 2 & 3 \\
\hline Source A & $80 \mathrm{kVp}$ & $100 \mathrm{kVp}$ & $140 \mathrm{kVp}$ \\
Exposure of source A & $500 \mathrm{mAs}$ & $250 \mathrm{mAs}$ & $75 \mathrm{mAs}$ \\
Source B & $140 / \mathrm{Sn} \mathrm{kVp}$ & $140 / \mathrm{Sn} \mathrm{kVp}$ & $80 \mathrm{kVp}$ \\
Exposure of source B & $193 \mathrm{mAs}$ & $193 \mathrm{mAs}$ & $413 \mathrm{mAs}$ \\
\hline
\end{tabular}

Table 4-5: Machine parameters used during experimental acquisition. A $0.8 \mathrm{~mm}$ Sn filter is present in the $140 \mathrm{kVp}$ source (i.e., noted $140 / \mathrm{Sn} \mathrm{kVp}$ ) for the first and second energy couples. Note that these are not clinical protocols.

spectra of interest (i.e., 80, 100, 140 and $140 / \mathrm{Sn} \mathrm{kVp}$ ), theoretical values of $\Gamma$ of each energy pair are plotted as a function of the atomic number of elements. As shown in figure $4-4$ for all energy pairs, $\Gamma$ is bijective with $Z$ for $1 \leq Z \leq 38$. Since the electron cross section is bijective with $Z$ over $1 \leq Z \leq 52$ (see figure 4-1), the domain where both functions are bijective is therefore $Z \in[1,38]$. The $Z_{\text {med }}$ value for ICRP tissues ranges from 6.26 to 13.52 (see table 4-2) and from 6.04 to 13.51 for the calibration phantom (see table 4-1).
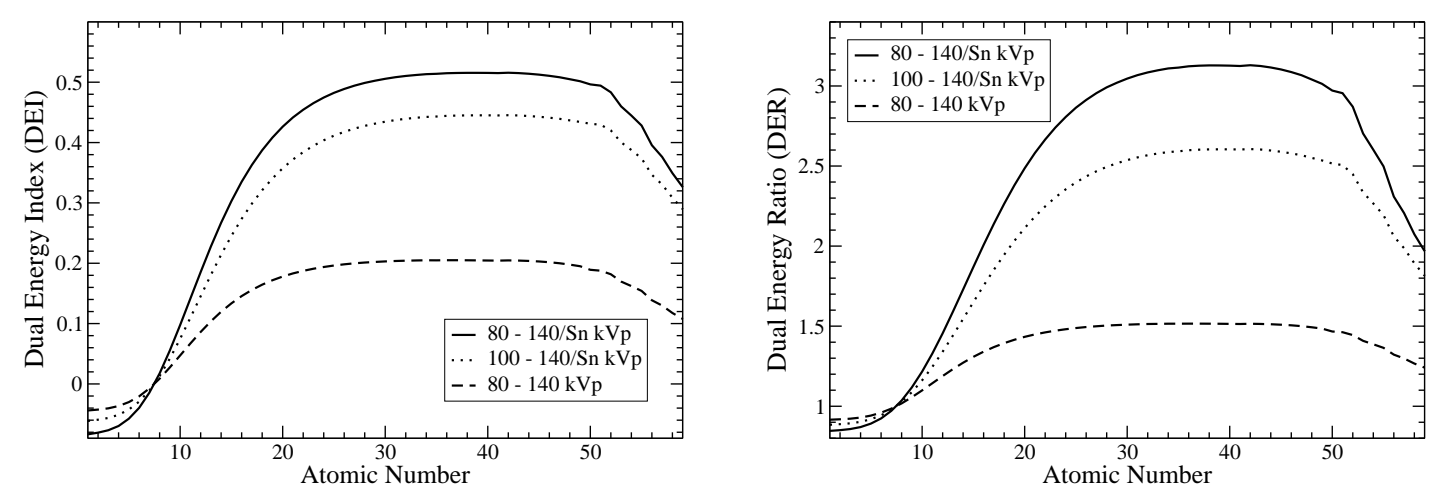

Figure 4-4: Behavior of the DEI (left) and DER (right) as a function the atomic number for elements. The data is obtained using XCOM cross sections averaged over the spectra. 

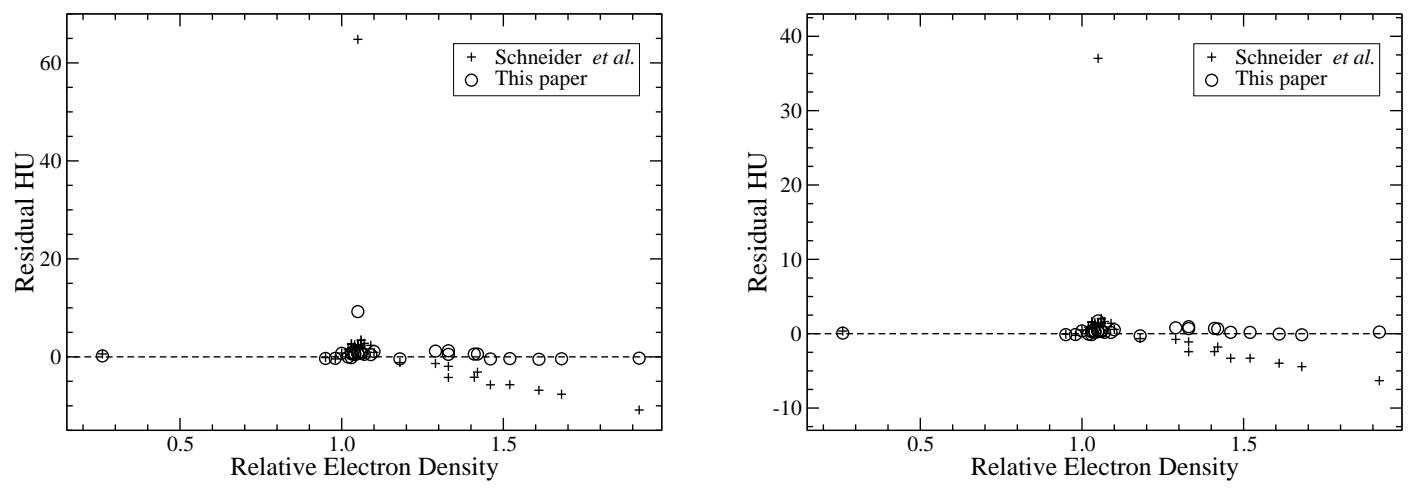

Figure 4-5: A theoretical comparison of residual HU errors between the model used by Schneider et al. and the present model. The spectra used to calculate theoretical $\mathrm{HU}$ values are a $80 \mathrm{kVp}$ spectrum (left) and a $140 \mathrm{kVp}$ spectrum (right).

\section{SECT stoichiometric calibration}

The residual HU error, defined as $\hat{\mathrm{HU}}-\mathrm{HU}$, is evaluated for different tissue composition using both Schneider et al.'s and the present SECT formalism. Figure 4-5 shows the residual HU error of ICRP tissues obtained from the stoichiometric calibration simulated with the Gammex 467 tissue compositions. Note than the order of the fit used in the present method is $M=6$ (as in equation 4.23). The present method is more accurate in predicting the HU of the ICRP tissues than the one used by Schneider et al., particularly for the thyroid where the difference goes from 65 to $9.3 \mathrm{HU}$ for $80 \mathrm{kVp}$ and from 37 to $1.7 \mathrm{HU}$ for $140 \mathrm{kVp}$. In addition, a systematic trend is observed in the bone region with the model used by Schneider et al., leading to errors up to about $11 \mathrm{HU}$ and $7 \mathrm{HU}$ for 80 and $140 \mathrm{kVp}$ respectively, while the present formalism yields residual errors less than $\pm 1.3 \mathrm{HU}$ and $\pm 0.8 \mathrm{HU}$ for the same energies (except for the thyroid). 


\section{DECT stoichiometric calibration}

Using the DECT calibration formalism for all three energy pairs, mean absolute errors of $0.026 \%$ and $0.057 \%$ for the ED and the EAN are obtained using the calibration phantom. The extension of the calibration to the ICRP tissues with the match of 100 and 140/Sn kVp spectra is found to yield optimal results and is shown in figure 4-6. Note that the error bars in figure 4-6 are calculated from analyzing the non statistical component of the uncertainty on $Z_{\text {med }}$, which by definition varies with energy. Although the thyroid presents higher relative errors because of its content in iodine, all other tissues stay under approximately $\pm 0.2 \%$ for both extracted quantities, with mean absolute errors of $0.08 \%$ and $0.17 \%$ for the ED and the EAN respectively. Comparatively, the study of Yang et al. [52] reported these mean absolute errors to be $0.16 \%$ and $0.79 \%$ for the theoretical evaluation of the DECT formalism of Bazalova et al. [3] with the 100 and $140 \mathrm{kVp}$ spectra. The results shown in figure $4-6$ are obtained with $\Gamma=\mathrm{DEI}$ and $K=6$ (as in equation 4.30). However, choosing $\Gamma=$ DER yields similar levels of accuracy.

\section{The problem of the thyroid}

As shown in figure 4-6, the error bars include all possible deviation of the estimated parameter (i.e., ED or EAN) from its actual value at the exception of the thyroid. As reported in several studies (e.g., see ref. [51, 52]), the thyroid is difficult to model due to its content in iodine which makes it highly sensitive to the energy spectrum due to the photoelectric effect dominance for low energies. While residual HU errors are present for the thyroid during the SECT calibration method (see figure 4-5), they also exists during the DECT stoichiometric calibration 

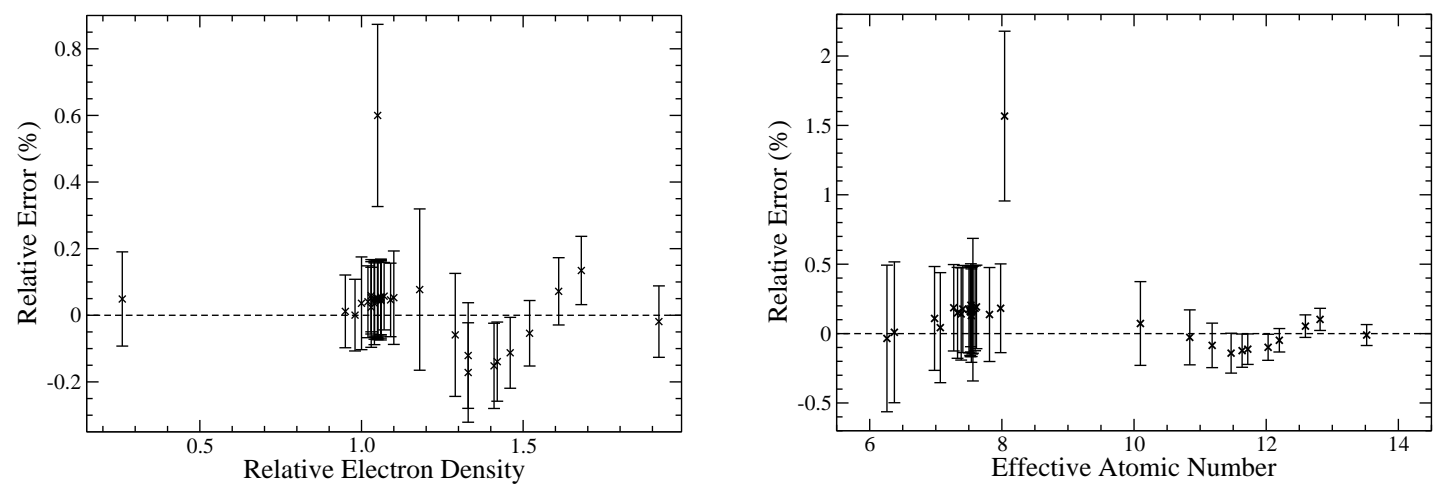

Figure 4-6: Relative errors in ED (left) and EAN (right) using the DECT formalism of theoretical HU values for 34 ICRP tissues. The error bars are non statistical uncertainties, i.e., $\Delta \rho_{e}$ is calculated with equation 4.50 and $\Delta Z_{\text {med }}$ is extracted from table $4-2$.

and cannot be explained by the non statistical errors shown in figure 4-6. The explanation of this discrepency resides in the relation between $\Gamma$ and $Z$. Indeed, the assumption that $Z_{\text {med }}$ is independent of energy allows establishing the equivalence $\Gamma \equiv \Gamma(Z) \leftrightarrow Z \equiv Z(\Gamma)$. In reality, this yields inconsistencies in performing the inversion of the function $\Gamma(Z)$, as done to obtain equation 4.30, and the thyroid is found to be the worse case. Figure 4-7 shows the non compliance of the thyroid to the $\Gamma-Z$ relation. Therefore one can conclude from the results of figure $4-6$ that the accuracy on the ED and EAN measurements cannot be better than $0.6 \%$ and $1.5 \%$ respectively. It is worth mentioning that for the other ICRP tissues, the agreement is acceptable within predicted uncertainties.

\subsubsection{Experimental measurements}

\section{SECT measurements}

While the model of Schneider et al. remains an excellent method of calibration, the performance of the present formalism with experimental measurements is slightly 


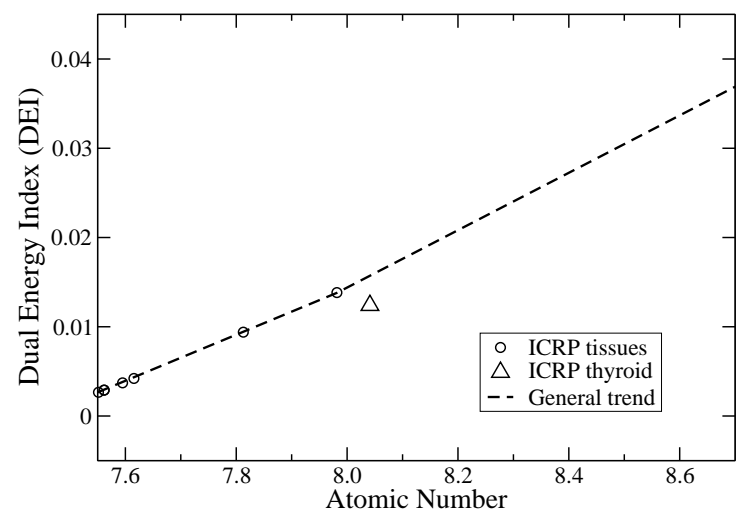

Figure 4-7: A theoretical comparison of the DEI between the ICRP tissues and the thyroid for the energy couple 100-140/Sn kVp.

superior in terms of accuracy. As shown in table 4-6, the global uncertainty on HU is systematically smaller with the present formalism versus Schneider's, except for 140/Sn kVp, yielding global uncertainties ranging from 4 to 9 HU over all spectra studied. These results are consistent with the manufacturer's specification of \pm 4 HU for water. While these levels of uncertainty for experimental measurements are significantly higher than for the theoretical framework, they are mostly affected by the impact of noise and artefacts. In a more clinical situation where voxel information is used (rather than VOI-averaged), the noise component dominates the uncertainty and the type of reconstruction algorithm is a determining factor. Table 4-7 shows standard deviations of HU in several VOI using two different reconstruction algorithms: 1) Filtered Back Projection (FBP), and 2) Sinogram Affirmed Iterative (SAFIRE). A comparison between tables $4-6$ and 4-7 demonstrates that the uncertainty increases by a factor of about 6-10 for water-like materials with the FBP algorithm, while a factor of about 4-8 is observed with SAFIRE. 


\begin{tabular}{ccc}
\hline Tube potential $(\mathrm{kVp})$ & Schneider et al. & This paper \\
\hline 80 & 12 & 9 \\
100 & 10 & 5 \\
140 & 7 & 5 \\
$140 / \mathrm{Sn}$ & 2 & 4 \\
\hline
\end{tabular}

Table 4-6: HU uncertainties for the SECT experimental calibration ( $1 \sigma$ level).

\section{DECT stoichiometric calibration}

Table 4-8 summarizes the RMS errors on all estimated physical parameters for the calibration phantom with the present formalism using the theoretical framework and experimental measurements. More specifically, figure 4-8 shows the performance of the formalism to extract different physical parameters of the Gammex 467 materials with the optimal energy couple (i.e., 100-140/Sn kVp). The reported uncertainties include statistical and non statistical uncertainty components (i.e., related to the definition of $Z_{\mathrm{med}}$ ). The mean absolute error of the measured ED and EAN are respectively $(0.3 \pm 0.4) \%$ and $(1.6 \pm 2.0) \%$. The mean absolute error of the measured $I$-value and the proton stopping power $(216 \mathrm{MeV})$ are respectively $(4.1 \pm$ $2.7) \%$ and $(0.5 \pm 0.4) \%$. Comparatively, the formalism of Bazalova et al. [3] yielded mean absolute errors of $(1.8 \pm 1.6) \%$ and $(2.8 \pm 2.6) \%$ for ED and EAN measurements respectively under similar conditions. Note that for higher $I$-values, the errors in figure 4-8 are significantly larger than the uncertainties since the estimator of $I$ as a function of $Z$ (i.e., see equation 4.21) is performed using ICRP tissue compositions only, i.e., with the absence of the calibration phantom materials. 


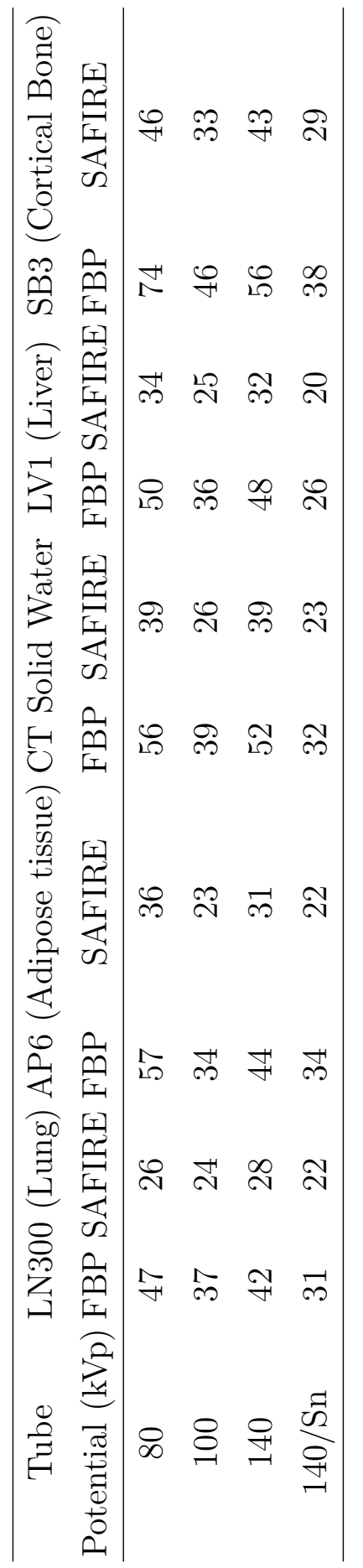

Table 4-7: Impact on the standard deviation of the HU for filtered back projection (FBP) and SAFIRE reconstruction processes. The third level of iteration of SAFIRE is used. 
The uncertainty on the estimation of the ED and the EAN is summarized in table 4-9 for a few calibration phantom materials. The chosen materials cover the range of the classical HU-ED curve: lung, adipose tissue, water and cortical bone. Uncertainties in ED range from 0.001 to 0.006 (relative to water), while the uncertainty on the EAN range from 0.01 to 1.5. While no clear trend exists for the uncertainty on the ED, the uncertainty on the EAN is high for low $Z_{\text {med }}$ and low for high $Z_{\text {med }}$. This trend is also observed in figure $4-8$.

While the comparison of the theoretical and experimental results with the formalism of Bazalova conclusively demonstrates the high accuracy of the present formalism, this could potentially be explained by several differences in the methods. While both formalisms are mathematically similar (i.e., the functions $F(Z)$ and $G(Z)$ of Bazalova are polynomials fitted from XCOM data), the present formalism uses a rigorous definition of the atomic number rather than Mayneord's, which seems to explain the better accuracy. Moreover, the present method has the advantage to be independent of the knowledge of the spectrum, once the EANs are calculated, as the constants of the polynomial functions used in the formalism are found during the stoichiometric calibration. This seems to yield a better agreement with measurements than the use of spectral information. While one could argue that the present method relies on spectrum information to determine the EAN data, the spectrum has a small impact on the value of $Z_{\text {med }}$, as shown in figure 4-2 and tables $4-1$ and 4-2, and therefore one could use software to estimate the spectrum shape from a basic description of the x-ray tube, as the anode angle and the target material (e.g., see ref. [34]). In addition, the present method does not require empirical beam 

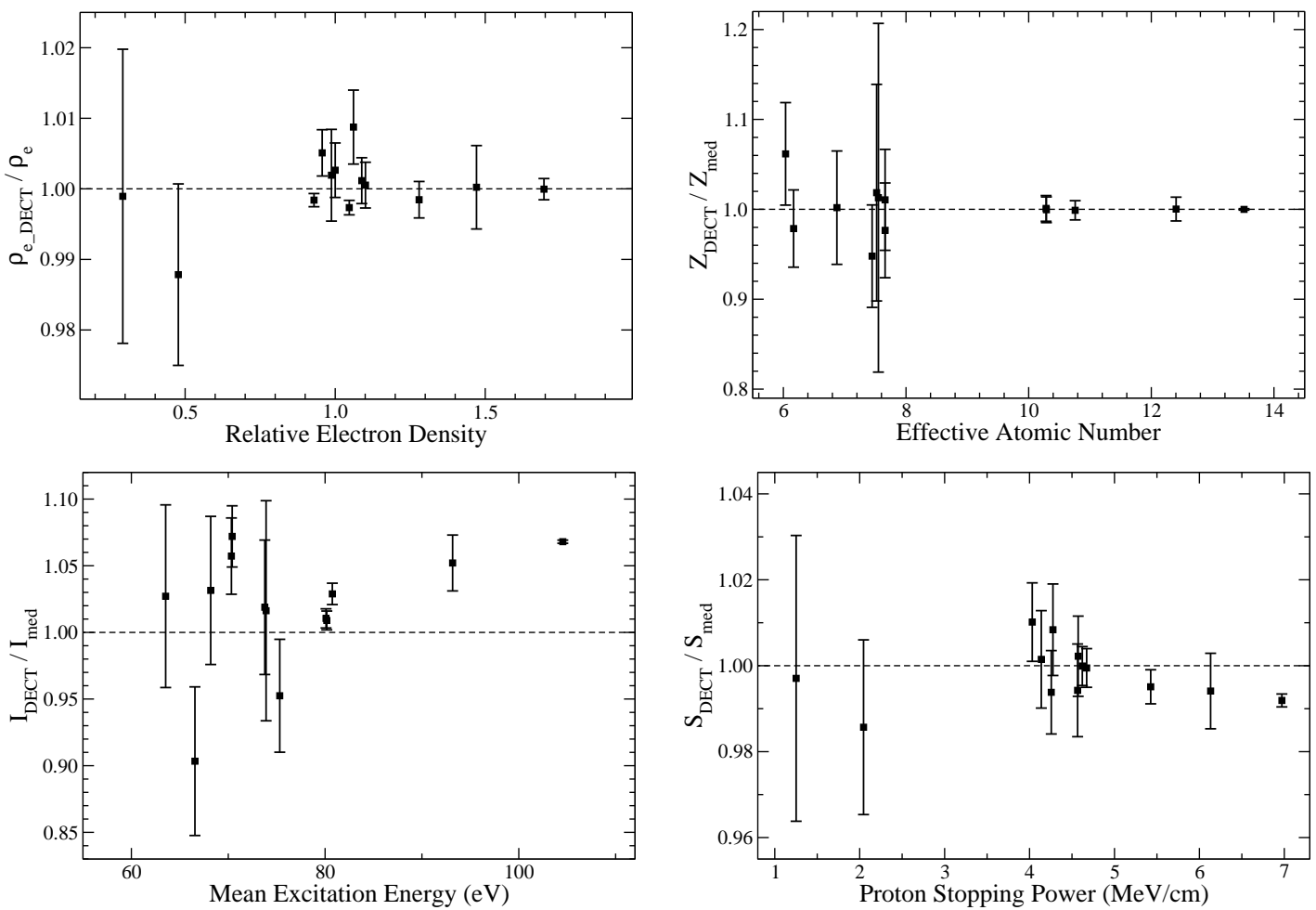

Figure 4-8: The ratios of calculated to known relative electron densities, EANs, mean excitation energies and proton stopping powers for the 100-140/Sn kVp energy couple. Note that the proton kinetic energy is $216 \mathrm{MeV}$. The reported uncertainties include statistical and non statistical components.

hardening corrections, such as in Bazalova's method. Finally, another issue that can explain discrepancies between both methods is the accuracy of composition data, for which the role on the method's performance remains unclear and relies on the manufacturer.

\subsubsection{Heavy-ion range uncertainties}

To evaluate the clinical impact of the formalism to radiation therapy, one direct application is heavy-ion therapy. Using Bethe's formula (see equation 4.10), one 


\begin{tabular}{llllllrrrr}
\hline & \multicolumn{3}{c}{ Theoretical } & \multicolumn{4}{c}{ Experimental } \\
Energy couple & $\rho_{e}$ & $Z_{\text {med }}$ & $I_{\text {med }}$ & $S$ & $\rho_{e}$ & $Z_{\text {med }}$ & $I_{\text {med }}$ & $S$ \\
\hline $80-140 / \mathrm{Sn} \mathrm{kVp}$ & 0.13 & 0.34 & 1.17 & 0.17 & 0.60 & 3.58 & 5.01 & 0.78 \\
$100-140 / \mathrm{Sn} \mathrm{kVp}$ & 0.13 & 0.30 & 1.17 & 0.17 & 0.46 & 2.50 & 4.86 & 0.67 \\
$80-140 \mathrm{kVp}$ & 0.20 & 0.42 & 1.16 & 0.21 & 0.63 & 4.26 & 5.56 & 0.87 \\
\hline
\end{tabular}

Table 4-8: Experimental RMS errors (in \%) of the calibration phantom's physical parameters determined experimentally.

\begin{tabular}{ccc}
\hline Material & $\sigma_{\rho_{e}}$ & $\sigma_{Z_{\text {med }}}$ \\
\hline LN300 (Lung) & 0.006 & 1.5 \\
AP6 (Adipose tissue) & 0.001 & 0.3 \\
CT Solid Water & 0.006 & 0.4 \\
LV1 (Liver) & 0.006 & 0.4 \\
SB3 (Cortical Bone) & 0.003 & 0.01 \\
\hline
\end{tabular}

Table 4-9: Experimental uncertainties on the electron density and the EAN for the 100-140/Sn kVp energy couple.

can estimate the stopping power from ED and EAN measurements, converting EAN into an estimation of the $I$-value using equation 4.21. The uncertainty analysis is performed based on the equations in section II.H. Figure 4-9 shows the trend of the uncertainty of the range in water based on the results of the stoichiometric calibration performed on experimental measurements. Three particles are studied: protons, helium ions and carbon ions, with both ions fully-ionized. The energy of the ions are chosen such that the maximum range is $30 \mathrm{~cm}$ and the continuous slowing down approximation (CSDA) is used to estimate the range numerically. Using the uncertainty levels reported during the stoichiometric calibration (i.e., table 4-6), the 
ideal uncertainty on the range is less than $0.16 \mathrm{~mm}$ for protons, less than $0.09 \mathrm{~mm}$ for helium ions and less than $0.07 \mathrm{~mm}$ for carbon ions.

Despite that these levels are clinically unrealistic since they neglect the presence of noise, they can be used as a basis to estimate uncertainties in clinical situations. Using the ratio between results in table 4-7 and table 4-6 for Solid Water at $100 \mathrm{kVp}$ and $140 / \mathrm{Sn} \mathrm{kVp}$, the $\mathrm{HU}$ uncertainties in clinical situations are higher by a factor of about 8 and 6 times than during the calibration, this for the FBP and SAFIRE algorithms respectively. As discussed in section II.H, one can get a good estimation of the uncertainty on physical parameters by multiplying the ideal uncertainty obtained from stoichiometric calibration measurements with these factors. This yields

to an estimation on the clinical range uncertainty with the FBP algorithm of less than about $1.3 \mathrm{~mm}, 0.7 \mathrm{~mm}$ and $0.6 \mathrm{~mm}$ for protons, helium ions and carbon ions respectively. Using the uncertainties reported with the SAFIRE algorithm, the clinical range is less than about $1.0 \mathrm{~mm}, 0.5 \mathrm{~mm}$ and $0.4 \mathrm{~mm}$ for protons, helium ions and carbon ions respectively. The estimations for protons are significantly lower than the 1-3 mm reported by Schaffner and Pedroni[38], which is based on the method of Schneider et al.

\subsection{Summary}

To implement the DECT stoichiometric calibration method accurately, here is a list of steps to follow:

1. Choose a phantom with tissue substitutes of known compositions and physical densities, and calculate the value of $Z_{\text {med }}$ of every materials according to the 


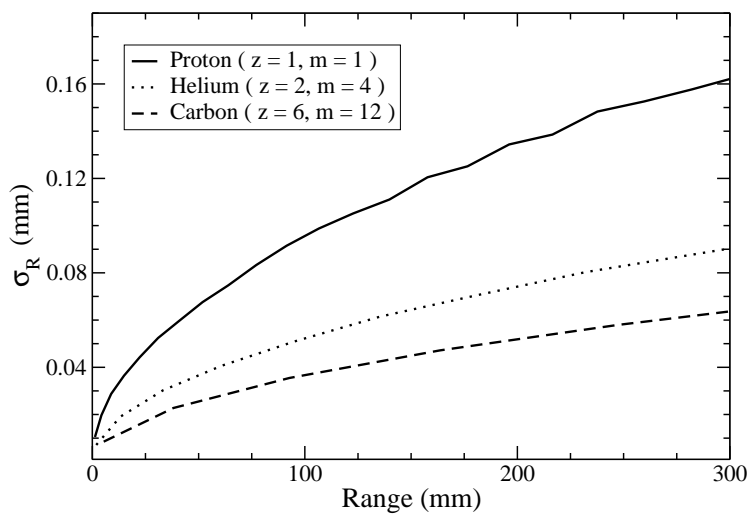

Figure 4-9: Ideal uncertainty on the range of protons, helium ions and carbon ions in water as a function of the range of the beam.

definition of equation 4.18. Choose the compositions to be such that the ranges of $Z_{\text {med }}$ and $\rho_{e}$ cover the ones of the ICRP tissues reported in table 4-2.

2. Scan these tissue substitutes with the DECT used for radiotherapy treatment planning to obtain the $\mathrm{HU}$ and convert them to values of reduced HU pairs (i.e., $u_{L}$ and $u_{H}$ ) with equation 4.28 .

3. Calculate the index $\Gamma$ (either DEI or DER) of every tissue substitute using equation 4.29. Find the least square solution of the coefficients $c_{k}$ with the matrix system of equation 4.31, given by equation 4.33. The estimator of the EAN in clinical situations is then calculated as in equation 4.34 .

4. Proceed the SECT stoichiometric calibration for both energies using the series of reduced $\mathrm{HU}$ for both energies (i.e., $u_{L}$ and $u_{H}$ ). Find the least square solution of the coefficient $b_{m}$ with the matrix system of equation 4.24 , given by equation 4.26. The estimator of the relative ED is then calculated with equations 4.35-4.36. 
5. For stopping power estimations, find the estimator of the mean excitation energy from $\hat{Z}_{\text {med }}$ with the relation stated by equation 4.21 .

6. To perform the variance analysis, follow the equations stated in section II.H.

\subsection{Acknowledgements}

The authors are grateful to Siemens and Philips for kindly providing spectrum information. The authors are also grateful to Gammex for kindly providing material information. One author (A. Bourque) wishes to acknowledge the financial support by the Ministère de la santé et des services sociaux du Québec. We wish to acknowledge Danis Blais for his involvment during experimental measurements.

\section{Appendix A: derivation of the uncertainty on the effective atomic number and the electron density}

During these derivations, it is implicit that $u_{\mathrm{L}}=u_{\mathrm{L}, \operatorname{med}}, u_{\mathrm{H}}=u_{\mathrm{H}, \mathrm{med}}$ and $\Gamma=\Gamma_{\text {meas }}$. Using the rule of error propagation on equation 4.34 , the variance of $\hat{Z}$ is given by

$$
\begin{aligned}
\sigma_{\hat{Z}}^{2} & \equiv \operatorname{VAR}(\hat{Z}) \\
& \approx\left[\frac{\partial \hat{Z}}{\partial \Gamma}\right]^{2} \operatorname{VAR}(\Gamma) \\
& \approx\left[\sum_{k=1}^{K}(k-1) \hat{c}_{k} \Gamma^{k-2}\right]^{2}\left[\left(\frac{\partial \Gamma}{\partial u_{\mathrm{L}}}\right)^{2} \sigma_{\mathrm{L}}^{2}+\left(\frac{\partial \Gamma}{\partial u_{\mathrm{H}}}\right)^{2} \sigma_{\mathrm{H}}^{2}\right] .
\end{aligned}
$$

with $\sigma_{\mathrm{L}}^{2}=\operatorname{VAR}\left(u_{\mathrm{L}}\right)$ and $\sigma_{\mathrm{H}}^{2}=\operatorname{VAR}\left(u_{\mathrm{H}}\right)$. Since $\Gamma$ stands for two definitions (i.e., DEI or DER, see equation 4.29), one writes

$$
\frac{\partial \Gamma}{\partial u_{\mathrm{L}}}=\left\{\begin{array}{cl}
\frac{2 u_{\mathrm{H}}}{\left(u_{\mathrm{L}}+u_{\mathrm{H}}\right)^{2}} & \text { for } \Gamma=\text { DEI } \\
\frac{1}{u_{\mathrm{H}}} & \text { for } \Gamma=\mathrm{DER}
\end{array}\right.
$$


and

$$
\frac{\partial \Gamma}{\partial u_{\mathrm{H}}}=\left\{\begin{array}{cl}
-\frac{2 u_{\mathrm{L}}}{\left(u_{\mathrm{L}}+u_{\mathrm{H}}\right)^{2}} & \text { for } \Gamma=\mathrm{DEI} \\
-\frac{u_{\mathrm{L}}}{u_{\mathrm{H}}^{2}} & \text { for } \Gamma=\mathrm{DER}
\end{array} .\right.
$$

Therefore equations A1 becomes

$$
\sigma_{\hat{Z}} \approx \begin{cases}\frac{2\left|\sum_{k=1}^{K}(k-1) \hat{c}_{k} \Gamma^{k-2}\right| \sqrt{u_{\mathrm{H}}^{2} \sigma_{\mathrm{L}}^{2}+u_{\mathrm{L}}^{2} \sigma_{\mathrm{H}}^{2}}}{\left(u_{\mathrm{L}}+u_{\mathrm{H}}\right)^{2}} & \text { for } \Gamma=\mathrm{DEI} \\ \frac{\left|\sum_{k=1}^{K}(k-1) \hat{c}_{k} \Gamma^{k-2}\right| \sqrt{u_{\mathrm{H}}^{2} \sigma_{\mathrm{L}}^{2}+u_{\mathrm{L}}^{2} \sigma_{\mathrm{H}}^{2}}}{u_{\mathrm{H}}^{2}} & \text { for } \Gamma=\mathrm{DER}\end{cases}
$$

The variance of $\hat{\rho}_{e}$ can then be written as follows

$$
\begin{aligned}
\sigma_{\hat{\rho}_{e}}^{2} & \equiv \operatorname{VAR}\left(\hat{\rho}_{e}\right) \\
& \approx\left[\frac{\partial \hat{\rho}_{e}}{\partial u_{\mathrm{L}}}\right]^{2} \operatorname{VAR}\left(u_{\mathrm{L}}\right)+\left[\frac{\partial \hat{\rho}_{e}}{\partial u_{\mathrm{H}}}\right]^{2} \operatorname{VAR}\left(u_{\mathrm{H}}\right) \\
& \approx \frac{1}{4}\left[\frac{\partial \hat{\rho}_{e, L}}{\partial u_{\mathrm{L}}}+\frac{\partial \hat{\rho}_{e, H}}{\partial u_{\mathrm{L}}}\right]^{2} \sigma_{\mathrm{L}}^{2}+\frac{1}{4}\left[\frac{\partial \hat{\rho}_{e, L}}{\partial u_{\mathrm{H}}}+\frac{\partial \hat{\rho}_{e, H}}{\partial u_{\mathrm{H}}}\right]^{2} \sigma_{\mathrm{H}}^{2}
\end{aligned}
$$

The derivative of $\hat{\rho}_{e, \mathrm{~L} / \mathrm{H}}$ with respect to $u_{\mathrm{L}}$ is given by

$$
\begin{aligned}
\frac{\partial \hat{\rho}_{e, \mathrm{~L} / \mathrm{H}}}{\partial u_{\mathrm{L}}} & =\frac{\frac{\partial u_{\mathrm{L} / \mathrm{H}}}{\partial u_{\mathrm{L}}}}{\sum_{m=1}^{M} \hat{b}_{\mathrm{L} / \mathrm{H}, m} \hat{Z}^{m-1}}-\frac{u_{\mathrm{L} / \mathrm{H}}\left[\sum_{m=1}^{M}(m-1) \hat{b}_{\mathrm{L} / \mathrm{H}, m} \hat{Z}^{m-2}\right] \frac{\partial \hat{Z}}{\partial u_{\mathrm{L}}}}{\left[\sum_{m=1}^{M} \hat{b}_{\mathrm{L} / \mathrm{H}, m} \hat{Z}^{m-1}\right]^{2}} \\
& =\frac{\frac{\partial u_{\mathrm{L} / \mathrm{H}}}{\partial u_{\mathrm{L}}}}{\sum_{m=1}^{M} \hat{b}_{\mathrm{L} / \mathrm{H}, m} \hat{Z}^{m-1}}-\frac{u_{\mathrm{L} / \mathrm{H}}\left[\sum_{m=1}^{M}(m-1) \hat{b}_{\mathrm{L} / \mathrm{H}, m} \hat{Z}^{m-2}\right]\left[\sum_{k=1}^{K}(k-1) \hat{c}_{k} \Gamma^{k-2}\right] \frac{\partial \Gamma}{\partial u_{\mathrm{L}}}}{\left[\sum_{m=1}^{M} \hat{b}_{\mathrm{L} / \mathrm{H}, m} \hat{Z}^{m-1}\right]^{2}} \\
& =\frac{\hat{\rho}_{e, \mathrm{~L} / \mathrm{H}}}{u_{\mathrm{L} / \mathrm{H}}} \frac{\partial u_{\mathrm{L} / \mathrm{H}}}{\partial u_{\mathrm{L}}}-\frac{\hat{\rho}_{e, \mathrm{~L} / \mathrm{H}}^{2}}{u_{\mathrm{L} / \mathrm{H}}} B_{\mathrm{L} / \mathrm{H}} \frac{\partial \Gamma}{\partial u_{\mathrm{L}}},
\end{aligned}
$$

with $B_{\mathrm{L} / \mathrm{H}}$ given by

$$
B_{\mathrm{L} / \mathrm{H}} \equiv\left[\sum_{m=1}^{M}(m-1) \hat{b}_{\mathrm{L} / \mathrm{H}, m} \hat{Z}^{m-2}\right]\left[\sum_{k=1}^{K}(k-1) \hat{c}_{k} \Gamma^{k-2}\right] \text {. }
$$


By symmetry with equation $\mathrm{A} 6$, the derivative of $\hat{\rho}_{e, \mathrm{~L} / \mathrm{H}}$ with respect to $u_{\mathrm{H}}$ is given by

$$
\frac{\partial \hat{\rho}_{e, \mathrm{~L} / \mathrm{H}}}{\partial u_{\mathrm{H}}}=\frac{\hat{\rho}_{e, \mathrm{~L} / \mathrm{H}}}{u_{\mathrm{L} / \mathrm{H}}} \frac{\partial u_{\mathrm{L} / \mathrm{H}}}{\partial u_{\mathrm{H}}}-\frac{\hat{\rho}_{e, \mathrm{~L} / \mathrm{H}}^{2}}{u_{\mathrm{L} / \mathrm{H}}} B_{\mathrm{L} / \mathrm{H}} \frac{\partial \Gamma}{\partial u_{\mathrm{H}}} .
$$

Combining equation A5 with equations A6 and A8 yields

$$
\sigma_{\hat{\rho}_{e}}^{2} \approx \frac{1}{4}\left[\frac{\hat{\rho}_{e, \mathrm{~L}}}{u_{\mathrm{L}}}-\left[\frac{\hat{\rho}_{e, \mathrm{~L}}^{2}}{u_{\mathrm{L}}} B_{\mathrm{L}}+\frac{\hat{\rho}_{e, \mathrm{H}}^{2}}{u_{\mathrm{H}}} B_{\mathrm{H}}\right] \frac{\partial \Gamma}{\partial u_{\mathrm{L}}}\right]^{2} \sigma_{\mathrm{L}}^{2}+\frac{1}{4}\left[\frac{\hat{\rho}_{e, \mathrm{H}}}{u_{\mathrm{H}}}-\left[\frac{\hat{\rho}_{e, \mathrm{~L}}^{2}}{u_{\mathrm{L}}} B_{\mathrm{L}}+\frac{\hat{\rho}_{e, \mathrm{H}}^{2}}{u_{\mathrm{H}}} B_{\mathrm{H}}\right] \frac{\partial \Gamma}{\partial u_{\mathrm{H}}}\right]^{2} \sigma_{\mathrm{H}}^{2} .
$$

Defining

$$
A \equiv\left[\frac{\hat{\rho}_{e, \mathrm{~L}}^{2}}{u_{\mathrm{L}}} B_{\mathrm{L}}+\frac{\hat{\rho}_{e, \mathrm{H}}^{2}}{u_{\mathrm{H}}} B_{\mathrm{H}}\right]
$$

one can write

$$
\sigma_{\hat{\rho}_{e}}^{2} \approx \frac{1}{4}\left[\frac{\hat{\rho}_{e, \mathrm{~L}}}{u_{\mathrm{L}}}-A \frac{\partial \Gamma}{\partial u_{\mathrm{L}}}\right]^{2} \sigma_{\mathrm{L}}^{2}+\frac{1}{4}\left[\frac{\hat{\rho}_{e, \mathrm{H}}}{u_{\mathrm{H}}}-A \frac{\partial \Gamma}{\partial u_{\mathrm{H}}}\right]^{2} \sigma_{\mathrm{H}}^{2}
$$

and therefore equation A11 becomes

$$
\sigma_{\hat{\rho}_{e}} \approx\left\{\begin{array}{cl}
\frac{1}{2} \sqrt{\left[\frac{\hat{\rho}_{e, \mathrm{~L}}}{u_{\mathrm{L}}}-\frac{2 A u_{\mathrm{H}}}{\left(u_{\mathrm{L}}+u_{\mathrm{H}}\right)^{2}}\right]^{2} \sigma_{\mathrm{L}}^{2}+\left[\frac{\hat{\rho}_{e, \mathrm{H}}}{u_{\mathrm{H}}}+\frac{2 A u_{\mathrm{L}}}{\left(u_{\mathrm{L}}+u_{\mathrm{H}}\right)^{2}}\right]^{2}} \sigma_{\mathrm{H}}^{2} & \text { for } \Gamma=\mathrm{DEI} \\
\frac{1}{2} \sqrt{\left[\frac{\hat{\rho}_{e, \mathrm{~L}}}{u_{\mathrm{L}}}-\frac{A}{u_{\mathrm{H}}}\right]^{2} \sigma_{\mathrm{L}}^{2}+\left[\frac{\hat{\rho}_{e, \mathrm{H}}}{u_{\mathrm{H}}}+\frac{A u_{\mathrm{L}}}{u_{\mathrm{H}}^{2}}\right]^{2} \sigma_{\mathrm{H}}^{2}} & \text { for } \Gamma=\mathrm{DER}
\end{array}\right.
$$

Since $\hat{\rho}_{e}$ and $\hat{Z}$ are statistically correlated, their covariance is given by:

$$
\begin{aligned}
\sigma_{\hat{\rho}_{e}, \hat{Z}} & \equiv \operatorname{COVAR}\left(\hat{\rho}_{e}, \hat{Z}\right) \\
& \approx \frac{\partial \hat{\rho}_{e}}{\partial u_{\mathrm{H}}} \frac{\partial \hat{Z}}{\partial u_{\mathrm{H}}} \sigma_{\mathrm{H}}^{2}+\left(\frac{\partial \hat{\rho}_{e}}{\partial u_{\mathrm{H}}} \frac{\partial \hat{Z}}{\partial u_{\mathrm{L}}}+\frac{\partial \hat{\rho}_{e}}{\partial u_{\mathrm{L}}} \frac{\partial \hat{Z}}{\partial u_{\mathrm{H}}}\right) \operatorname{COVAR}\left(u_{\mathrm{L}}, u_{\mathrm{H}}\right)+\frac{\partial \hat{\rho}_{e}}{\partial u_{\mathrm{L}}} \frac{\partial \hat{Z}}{\partial u_{\mathrm{L}}} \sigma_{\mathrm{L}}^{2} \\
& \approx \frac{\partial \hat{\rho}_{e}}{\partial u_{\mathrm{H}}} \frac{\partial \hat{Z}}{\partial u_{\mathrm{H}}} \sigma_{\mathrm{H}}^{2}+\frac{\partial \hat{\rho}_{e}}{\partial u_{\mathrm{L}}} \frac{\partial \hat{Z}}{\partial u_{\mathrm{L}}} \sigma_{\mathrm{L}}^{2} .
\end{aligned}
$$


Note here that $u_{\mathrm{L}}$ and $u_{\mathrm{H}}$ are assumed statistically independent and therefore $\operatorname{COVAR}\left(u_{\mathrm{L}}, u_{\mathrm{H}}\right)=$ 0. For $\Gamma=\mathrm{DEI}$, equation A13 becomes

$$
\begin{aligned}
\sigma_{\hat{\rho}_{e}, \hat{Z}} \approx & \frac{-u_{\mathrm{L}}}{\left(u_{\mathrm{L}}+u_{\mathrm{H}}\right)^{2}}\left[\sum_{k=1}^{K}(k-1) \hat{c}_{k} \Gamma^{k-2}\right]\left[\frac{\hat{\rho}_{e, \mathrm{H}}}{u_{\mathrm{H}}}+\frac{2 A u_{\mathrm{L}}}{\left(u_{\mathrm{L}}+u_{\mathrm{H}}\right)^{2}}\right] \sigma_{\mathrm{H}}^{2} \\
& +\frac{u_{\mathrm{H}}}{\left(u_{\mathrm{L}}+u_{\mathrm{H}}\right)^{2}}\left[\sum_{k=1}^{K}(k-1) \hat{c}_{k} \Gamma^{k-2}\right]\left[\frac{\hat{\rho}_{e, \mathrm{~L}}}{u_{\mathrm{L}}}-\frac{2 A u_{\mathrm{H}}}{\left(u_{\mathrm{L}}+u_{\mathrm{H}}\right)^{2}}\right] \sigma_{\mathrm{L}}^{2},
\end{aligned}
$$

and for $\Gamma=$ DER, equation A13 becomes

$$
\begin{aligned}
\sigma_{\hat{\rho}_{e}, \hat{z}} \approx & -\frac{u_{\mathrm{L}}}{2 u_{\mathrm{H}}^{2}}\left[\sum_{k=1}^{K}(k-1) \hat{c}_{k} \Gamma^{k-2}\right]\left[\frac{\hat{\rho}_{e, \mathrm{H}}}{u_{\mathrm{H}}}+\frac{A u_{\mathrm{L}}}{u_{\mathrm{H}}^{2}}\right] \sigma_{\mathrm{H}}^{2} \\
& +\frac{1}{2 u_{\mathrm{H}}}\left[\sum_{k=1}^{K}(k-1) \hat{c}_{k} \Gamma^{k-2}\right]\left[\frac{\hat{\rho}_{e, \mathrm{~L}}}{u_{\mathrm{L}}}-\frac{A}{u_{\mathrm{H}}}\right] \sigma_{\mathrm{L}}^{2} .
\end{aligned}
$$

\section{Appendix B: derivation of heavy-ion stopping power uncertainties}

The variance on the stopping power is given by

$$
\begin{aligned}
\sigma_{\hat{S}}^{2} \equiv & \operatorname{VAR}(\hat{S}) \\
\approx & {\left[\frac{\partial \hat{S}}{\partial \rho_{e}}\right]^{2} \sigma_{\hat{\rho}_{e}}^{2}+\left[\frac{\partial \hat{S}}{\partial Z}\right]^{2} \sigma_{\hat{Z}}^{2}+2\left[\frac{\partial \hat{S}}{\partial \rho_{e}}\right]\left[\frac{\partial \hat{S}}{\partial Z}\right] \sigma_{\hat{\rho}_{e}, \hat{Z}} } \\
\approx & {\left[\frac{k_{0} z^{2}}{\beta^{2}}\left[\ln \left(\frac{2 m_{e} c^{2} \beta^{2}}{I\left(1-\beta^{2}\right)}\right)-\beta^{2}\right]\right]^{2} \sigma_{\hat{\rho}_{e}}^{2}+\left[-\frac{\rho_{e}}{I} \frac{k_{0} z^{2}}{\beta^{2}} \frac{\partial \hat{I}}{\partial Z}\right]^{2} \sigma_{\hat{Z}}^{2} } \\
& +2\left[\frac{k_{0} z^{2}}{\beta^{2}}\left[\ln \left(\frac{2 m_{e} c^{2} \beta^{2}}{I\left(1-\beta^{2}\right)}\right)-\beta^{2}\right]\right]\left[-\frac{\rho_{e}}{I} \frac{k_{0} z^{2}}{\beta^{2}} \frac{\partial \hat{I}}{\partial Z}\right] \sigma_{\hat{\rho}_{e}, \hat{Z}} \\
\approx & {\left[\frac{\hat{S}}{\rho_{e}}\right]^{2} \sigma_{\hat{\rho}_{e}}^{2}+\left[-\frac{\rho_{e}}{\hat{I}} \frac{k_{0} z^{2}}{\beta^{2}} \frac{\partial \hat{I}}{\partial Z}\right]^{2} \sigma_{\hat{Z}}^{2}-2\left[\frac{\hat{S}}{\rho_{e}}\right]\left[\frac{\rho_{e}}{\hat{I}} \frac{k_{0} z^{2}}{\beta^{2}} \frac{\partial \hat{I}}{\partial Z}\right] \sigma_{\hat{\rho_{e}}, \hat{Z}} . }
\end{aligned}
$$




\section{Appendix C: derivation of the uncertainty on heavy-ion range}

Assuming the heavy ion to cross $N$ voxels of dimensions $\Delta x_{i}$ (in the direction of the beam), the average energy loss by the particle is approximately equal to

$$
\Delta E \approx \sum_{i=1}^{N} \Delta x_{i} S_{i},
$$

with $S_{i}$ the stopping power in the $i^{\text {th }}$ voxel. For equally-size voxels, $\Delta x_{i} \equiv \Delta x$ and therefore

$$
\Delta E \approx N \Delta x\left[\frac{1}{N} \sum_{i=1}^{N} S_{i}\right]
$$

Let us choose the initial kinetic energy of the ion $E_{0}$ such that $\Delta E=E_{0}$. Hence, the estimated range is given by $\hat{R} \approx N \Delta x$ and therefore

$$
E_{0} \approx \hat{R}\left[\frac{1}{N} \sum_{i} S_{i}\right]
$$

hence

$$
\hat{R} \approx \frac{E_{0}}{\left[\frac{1}{N} \sum_{i} S_{i}\right]} .
$$

Therefore the variance on the estimated range is given by

$$
\begin{aligned}
\sigma_{\hat{R}}^{2} & \equiv \operatorname{VAR}(\hat{R}) \\
& \approx \frac{E_{0}^{2}}{\left[\frac{1}{N} \sum_{i} S_{i}\right]^{4}} \frac{1}{N^{2}} \sum_{i}^{N} \operatorname{VAR}\left(S_{i}\right) \\
& \approx \frac{\hat{R}^{2}}{\bar{S}^{2}} \frac{\sigma_{S}^{2}}{N} \\
& \approx \hat{R} \Delta x \frac{\overline{\sigma_{S}^{2}}}{\bar{S}^{2}} .
\end{aligned}
$$




\section{Chapter 5 \\ Conclusion}

In this study, a formalism was developed to extract the relative electron density and the effective atomic number from clinical DECT images. A novel definition of this last quantity was also proposed and used throughout this work. A relationship between $Z_{\text {med }}$ and the mean excitation energy was given to calculate stopping powers needed for proton and heavy ion therapy. As part of the DECT formalism, the SECT calibration was re-evaluated. Both theoretical and experimental frameworks show results equivalent or superior of what is found in the literature.

In clinical situations, the produced DECT images generally show a high level of noise and several artifacts. While such problems could affect the effectiveness of the formalism applied on image voxels, improvements in reconstruction algorithms could reduce the impact of noise and artifacts on the extracted parameters.

In this study, the advantage of an additional parameter, such as the effective atomic number, is clearly demonstrated for heavy ion therapy where the accuracy on the electron density is crucial for determining the range of the beam. While it was not directly addressed in this work, a strong impact is also expected in brachytherapy because of the dominance of the photoelectric effect in the keV range, which is governed by a strong dependence on the atomic number of the medium.

Moreover, the uncertainty analysis of the paper described in chapter 4 allows concluding that the accuracy on the range of various heavy ions is superior to what is 
reported in the literature by a factor of about 3 . In the near future, it is expected that more accurate dose calculation could be obtained using ED-EAN maps, potentially becoming available in treatment planning systems using the method developed in this work.

While most radiotherapy patients are treated with megavoltage x-rays, the real impact on the accuracy of dose calculation at such energies is yet to be determined. Based on the developments and current needs for accurate methods such as Monte Carlo, it is expected that converting calculated ED-EAN maps to well-characterized geometries will become essential for clinical practice. In the case where future studies demonstrate no significant advantage of the DECT for megavoltage beams, the present study remains useful to improve the SECT stoichiometric calibration for megavoltage beams, which is proven to be more accurate than the method of Schneider et al.

Lastly, I would like to emphasize the fact that tremendous work needs to be done to show all benefits of DECT for radiation therapy. Indeed, the specific application of the extraction of the electron density and the effective atomic number is only one among other several potential ones. Therefore, I hope that this research will serve as a basis in many further investigations to optimize the use of DECT in radiotherapy. 


\section{References}

[1] Anders Ahnesjö. Collapsed cone convolution of radiant energy for photon dose calculation in heterogeneous media. Medical Physics, 16:577-592, 1989.

[2] R. E. Alvarez and A. Macovski. Energy-selective Reconstructions in X-ray Computerized Tomography. Physics in Medicine and Biology, 21(5):733-744, 1976.

[3] M. Bazalova, J.-F. Carrier, L. Beaulieu, and F. Verhaegen. Dual-energy CTbased material extraction for tissue segmentation in Monte Carlo dose calculations. Physics in Medicine and Biology, 53(9):2439-2456, 2008.

[4] M. Bazalova, J.-F. Carrier, L. Beaulieu, and F. Verhaegen. Tissue segmentation in Monte Carlo treatment planning: A simulation study using dual-energy CT images. Radiotherapy and Oncology, 86(1):93-98, 2008.

[5] S. Bedwani, J.-F. Carrier, and H. Bouchard. SU,ÄêE,ÄêT,Äêf01: A Sensitivity Study of Tissue Characterization for Brachytherapy Monte Carlo Dose Calculation. Medical Physics, 40(6):320, 2013.

[6] M. J. Berger, J. H. Hubbell, S. M. Seltzer, J. Chang, J. S. Coursey, R. Sukumar, and D. S. Zucker. Xcom: Photon cross sections database. NIST Standard Reference Database, 8:87-3597, 1998.

[7] H. Bischel. Passage of charged particles through matter, pages 8-142-89. McGraw-Hill, New-York, 1972.

[8] H. Bouchard, J. Seuntjens, J.-F. Carrier, and I. Kawrakow. Ionization chamber gradient effects in nonstandard beam configurations. Medical Physics, 36:4654, 2009.

[9] J.T. Bushberg and J.M. Boone. The essential physics of medical imaging. Lippincott Williams \& Wilkins, 2011.

[10] N. Chandra and D. A. Langan. Gemstone detector: dual energy imaging via fast $\mathrm{kVp}$ switching. In Dual energy $C T$ in clinical practice, pages 35-41. Springer, 2011. 
[11] I. J. Chetty, B. Curran, J. E. Cygler, J. J. DeMarco, G. Ezzell, B. A. Faddegon, I. Kawrakow, P. J. Keall, H. Liu, C.-M. C. Ma, et al. Report of the AAPM Task Group No. 105: Issues associated with clinical implementation of Monte Carlo-based photon and electron external beam treatment planning. Medical Physics, 34:4818-4853, 2007.

[12] F. C. P. Du Plessis, C. A. Willemse, M. G. Lötter, and L. Goedhals. The indirect use of CT numbers to establish material properties needed for Monte Carlo calculation of dose distributions in patients. Medical Physics, 25:11951201, 1998.

[13] T. G. Flohr, C. H. McCollough, H. Bruder, M. Petersilka, K. Gruber, C. Süß, M. Grasruck, K. Stierstorfer, B. Krauss, R. Raupach, et al. First performance evaluation of a dual-source CT (DSCT) system. European Radiology, 16(2):256$268,2006$.

[14] H. Guan, F.-F. Yin, and J. H. Kim. Accuracy of inhomogeneity correction in photon radiotherapy from CT scans with different settings. Physics in Medicine and Biology, 47(17):N223-N231, 2002.

[15] G. N. Hounsfield. Computerized transverse axial scanning (tomography): Part 1. Description of system. British Journal of Radiology, 46(552):1016-1022, 1973.

[16] ICRU. Stopping powers for electrons and positrons. ICRU report 37, 1984.

[17] ICRU. Prescribing, recording and reporting photon beam therapy. ICRU Report 50, 1993.

[18] National Cancer Institute. http://www.cancer.gov/, June 2013.

[19] D. F. Jackson and D. J. Hawkes. X-ray attenuation coefficients of elements and mixtures. Physics Reports, 70(3):169-233, 1981.

[20] H. Jiang and H. Paganetti. Adaptation of GEANT4 to Monte Carlo dose calculations based on CT data. Medical Physics, 31:2811-2818, 2004.

[21] T. Johnson. Dual energy CT in clinical practice. Springer-Verlag Berlin Heidelberg, 2011.

[22] B. J. Kirby, J. R. Davis, J. A. Grant, and M. J. Morgan. Extracting material parameters from x-ray attenuation: a CT feasibility study using kilovoltage 
synchrotron x-rays incident upon low atomic number absorbers. Physics in Medicine and Biology, 48(20):3389-3409, 2003.

[23] G. Landry, B. Reniers, P. V. Granton, B. van Rooijen, L. Beaulieu, J. E. Wildberger, and F. Verhaegen. Extracting atomic numbers and electron densities from a dual source dual energy CT scanner: Experiments and a simulation model. Radiotherapy and Oncology, 100(3):375-379, 2011.

[24] A. H. Mahnken, S. Stanzel, and B. Heismann. Spectral $\rho$ Z-Projection Method for Characterization of Body Fluids in Computed Tomography: Ex Vivo Experiments 1. Academic Radiology, 16(6):763-769, 2009.

[25] S. R. Manohara, S. M. Hanagodimath, K. S. Thind, and L. Gerward. On the effective atomic number and electron density: A comprehensive set of formulas for all types of materials and energies above $1 \mathrm{keV}$. Nuclear Instruments and Methods in Physics Research Section B: Beam Interactions with Materials and Atoms, 266(18):3906-3912, 2008.

[26] W. V. Mayneord. The significance of the roentgen. Acta Internat. Union Against, 1937.

[27] S. M. Midgley. A parameterization scheme for the x-ray linear attenuation coefficient and energy absorption coefficient. Physics in Medicine and Biology, 49(2):307-325, 2004.

[28] S. M. Midgley. Feasibility study for a novel method of dual energy x-ray analysis. Physics in Medicine and Biology, 56(17):5599-5619, 2011.

[29] H. Paganetti. Range uncertainties in proton therapy and the role of monte carlo simulations. Physics in Medicine and Biology, 57(11):R99-R117, 2012.

[30] H. Paganetti, H. Jiang, K. Parodi, R. Slopsema, and M. Engelsman. Clinical implementation of full Monte Carlo dose calculation in proton beam therapy. Physics in Medicine and Biology, 53(17):4825-4853, 2008.

[31] M. Petersilka, H. Bruder, B. Krauss, K. Stierstorfer, and T. G. Flohr. Technical principles of dual source CT. European Journal of Radiology, 68(3):362-368, 2008.

[32] E. B. Podgorsak. Radiation physics for medical physicists. Springer-Verlag, Berlin, second edition, 2009. 
[33] E. B. Podgorsak et al. Radiation oncology physics: a handbook for teachers and students. International Atomic Energy Agency, 2005.

[34] G. Poludniowski, G. Landry, F. DeBlois, P. M. Evans, and F. Verhaegen. Spekcalc: a program to calculate photon spectra from tungsten anode x-ray tubes. Physics in Medicine and Biology, 54(19):N433, 2009.

[35] W. H. Press, S. A. Teukolsky, W. T. Vetterling, and B. P Flannery. Numerical recipes in $\mathrm{c}++.2002$.

[36] R. A. Rutherford, B. R. Pullan, and I. Isherwood. Measurement of effective atomic number and electron density using an EMI scanner. Neuroradiology, 11(1):15-21, 1976.

[37] M. Saito. Potential of dual-energy subtraction for converting CT numbers to electron density based on a single linear relationship. Medical Physics, 39:20212030, 2012.

[38] B Schaffner and E Pedroni. The precision of proton range calculations in proton radiotherapy treatment planning: experimental verification of the relation between CT-HU and proton stopping power. Physics in Medicine and Biology, 43(6):1579-1592, 1998.

[39] J. P. Schlomka et al. Experimental feasibility of multi-energy photon-counting K-edge imaging in pre-clinical computed tomography. Physics in medicine and biology, 53:4031-4047, 2008.

[40] U. Schneider, E. Pedroni, and A. Lomax. The calibration of CT Hounsfield units for radiotherapy treatment planning. Physics in Medicine and Biology, 41(1):111-124, 1996.

[41] W. Schneider, T. Bortfeld, and W. Schlegel. Correlation between CT numbers and tissue parameters needed for Monte Carlo simulations of clinical dose distributions. Physics in Medicine and Biology, 45(2):459-478, 2000.

[42] W. S. Snyder, M. J. Cook, E. S. Nasset, L. R. Karhausen, G. P. Howells, and I. H. Tipton. Report of the task group on reference man, volume 23. Pergamon Oxford, 1975.

[43] Canadian Cancer Society. http://www.cancer.ca/, June 2013. 
[44] W. Spiers. Effective atomic number and energy absorption in tissues. British Journal of Radiology, 19:52-63, 1946.

[45] M. L. Taylor, R. L. Smith, F. Dossing, and R. D. Franich. Robust calculation of effective atomic numbers: The Auto-Zeff software. Medical Physics, 39:1769$1778,2012$.

[46] SJ Thomas. Relative electron density calibration of CT scanners for radiotherapy treatment planning. British Journal of Radiology, 72(860):781-786, 1999.

[47] M. Torikoshi, T. Tsunoo, M. Sasaki, M. Endo, Y. Noda, Y. Ohno, T. Kohno, K. Hyodo, K. Uesugi, and N. Yagi. Electron density measurement with dualenergy x-ray CT using synchrotron radiation. Physics in Medicine and Biology, 48(5):673-685, 2003.

[48] T. Tsunoo, M. Torikoshi, Y. Ohno, K. Uesugi, and N. Yagi. Measurement of electron density in dual-energy x-ray CT with monochromatic $\mathrm{x}$ rays and evaluation of its accuracy. Medical Physics, 35(11):4924-4932, 2008.

[49] B. Vanderstraeten, P. W. Chin, M. Fix, A. Leal, G. Mora, N. Reynaert, J. Seco, M. Soukup, E. Spezi, W. De Neve, et al. Conversion of CT numbers into tissue parameters for Monte Carlo dose calculations: a multi-centre study. Physics in Medicine and Biology, 52(3):539-562, 2007.

[50] F. Verhaegen and S. Devic. Sensitivity study for CT image use in Monte Carlo treatment planning. Physics in Medicine and Biology, 50(5):937-946, 2005.

[51] J. F. Williamson, S. Li, S. Devic, B. R. Whiting, and F. A. Lerma. On twoparameter models of photon cross sections: Application to dual-energy CT imaging. Medical Physics, 33(11):4115-4129, 2006.

[52] M. Yang, G. Virshup, J. Clayton, X. R. Zhu, .R Mohan, and L. Dong. Theoretical variance analysis of single-and dual-energy computed tomography methods for calculating proton stopping power ratios of biological tissues. Physics in Medicine and Biology, 55(5):1343-1362, 2010. 